\title{
Ultrafast photoelectron spectroscopy near liquid water interfaces: The solvated electron
}

\author{
Dissertation \\ zur Erlangung des mathematisch-naturwissenschaftlichen Doktorgrades \\ "Doctor rerum naturalium" \\ der Georg-August-Universität Göttingen
}

vorgelegt von

Katrin Rita Siefermann

aus Oberkirch (Baden)

Göttingen, 2010 
Referent: Prof. Dr. Bernd Abel

Korreferent: Prof. Dr. Martin Suhm

Tag der mündlichen Prüfung: 09. Juli 2010 
The negative ion constitutes a new species of anion. It consists of a negative charge, an electron, surrounded by an envelope of solvent molecules.

Charles A. Kraus, 1908. 



\section{Acknowledgements}

I would like to express my deep and sincere gratitude to my supervisor, Prof. Dr. Bernd Abel. His wide knowledge and his logical way of thinking have been of great value for me. I also wish to thank him for supporting my scientific career and for giving me enough freedom for developing my own scientific creativity.

I would like to show my gratitude to Prof. Dr. Martin Suhm for several valuable comments on my work, and for the membership in his Physical \& Chemical Graduate School Göttingen (GRK 782). I particularly wish to thank the GRK 782 for financial support of my project.

I would like to thank my dear colleague Yaxing Liu for the great teamwork during the last years and for several valuable ideas and discussions. It was a pleasure working with you. My special thanks also go to Dr. Evgeny Lugovoy and Dr. Oliver Link as well as the entire Abel group.

During this work I have collaborated with many colleagues for whom I have great regard, and I wish to extend my thanks to: Dr. Manfred Faubel and Prof. Dr. Udo Buck for their help and support. I want to thank Ondrej Marsalek, Prof. Pavel Jungwirth, Dr. Michal Farnik, and Prof. Daniel M. Neumark for fruitful discussions. I also received a lot of valuable comments from Dr. Thomas Zeuch and Richard Forck.

I am grateful for the great technical support by the electronic workshop and the machine shop of our institute, as well as by Werner Noack and Lars Sölter.

I wish to express my warm and sincere thanks to all my friends who commented on the manuscript of this thesis: Dr. Andrew Crowther, Dr. Holger Schneider, Daniel Packwood, Juhyon Lee, Tyler Arbour, Verena Müller, Theresia Schnurr, and Josua Schinke.

It is my particular wish to thank all my friends from Göttingen, and from all over the world. They made the last couple of years to some of the best in my life.

I owe my loving thanks to my family: to my grandparents, my brothers and particularly to my mother for always giving me strength and hope. To her I dedicate this thesis. 



\section{Summary}

The nature of the solvated electron, which was first observed in liquid ammonia in 1864, continues to pose fundamental problems. In liquid water, the hydrated electron is essential to many physical, chemical, and biological processes. Despite its importance, the binding energy of the solvated electron in bulk water has not been measured experimentally, although there have been several attempts to extrapolate this quantity from cluster experiments, or to derive it from theory. Within this doctoral thesis, vertical binding energies (VBE) of hydrated electrons have been directly measured using liquid micro jet technology in vacuum and a table top high harmonic light source driven by a femtosecond laser system. The key feature to our experimental approach is generating solvated electrons by a short pulse of $267 \mathrm{~nm}$ light and recording photoelectron spectra using a time-delayed $38.7 \mathrm{eV}(32 \mathrm{~nm})$ high harmonic probe pulse. For different precursors and experimental conditions we are able to record photoelectron spectra with prominent emission features at $(3.3 \pm 0.1) \mathrm{eV}$ and $(1.6 \pm 0.1) \mathrm{eV}$, which we assign to the vertical binding energies of the internally solvated and the surface solvated state of the electron, respectively. Our results demonstrate the existence of surface solvated electrons at the water/gas interface with a lifetime longer than $100 \mathrm{ps}$.

These results define the energy scale of the solvated electron in liquid water, which is crucial information for many electron transfer processes in nature. This thesis discusses the concept of Resonant Dissociative Electron Attachment (RDEA). The key point in the RDEA concept is the comparison of electron acceptance windows of molecules in aqueous environments - which are resonances for electron transfer and attachment - with the energy scale of the hydrated electron. The concept may have far-reaching consequences for our understanding of radiation-induced DNA damage, since it suggests that the class of partially hydrated electrons may be responsible for a large portion of it. Similarly, the concept allows a promising new insight into electron attachment to chlorine-containing molecules on the surface of water ice particles. This electron attachment is currently discussed as a crucial step in a reaction mechanism leading to the formation of the Antarctic ozone hole The RDEA concept may in general enrich our understanding of many processes involving electrons in aqueous environments. 



\section{Contents}

1 Introduction 11

1.1 Structure and dynamics of the hydrated electron . . . . . . . . . . . 14

1.1.1 Two-photon ionization of liquid water . . . . . . . . . . 15

1.1.2 Photodetachment from $\left[\mathrm{Fe}(\mathrm{CN})_{6}\right]^{4-}$ in aqueous solution . . . 16

1.2 Anionic water clusters and electrons at water interfaces . . . . . . 17

1.3 Electron induced strand breaks in DNA . . . . . . . . . . . . . . 20

1.4 Cosmic ray driven electron induced ozone depletion . . . . . . . . . 22

2 Experimental approach $\quad 25$

2.1 Laser system . . . . . . . . . . . . . . . . . . . . 26

2.2 High harmonic radiation . . . . . . . . . . . . . . . . . . . . . . 29

2.2.1 Semiclassical three-step model . . . . . . . . . . . . . . . . 31

2.2.2 High harmonic light source . . . . . . . . . . . . . . . . . 34

2.3 Liquid phase photoelectron spectroscopy . . . . . . . . . . . . . 36

2.3.1 Liquid micro jet . . . . . . . . . . . . . . . . . . 37

2.3.2 Photoelectron spectroscopy on a liquid water surface . . . . 39

2.3.3 Time-resolved experiments . . . . . . . . . . . . . . . . 42

2.3.4 Surface sensitivity . . . . . . . . . . . . . . . 43

2.3.5 Photoelectron spectrometer and calibration . . . . . . . . . 45

3 Experimental strategy and results $\quad 49$

3.1 Surface solvated electron . . . . . . . . . . . . . . . . . . . . 49

3.2 Bulk solvated electron . . . . . . . . . . . . . . . . . 54

3.3 Relative concentrations of bulk and surface solvated electrons . . . . 58

4 Discussion $\quad 63$

4.1 Bulk solvated electrons . . . . . . . . . . . . . . . . 63

4.2 Surface solvated electrons _. . . . . . . . . . . . . . . 66

4.3 Resonant dissociative electron attachment . . . . . . . . . . . . 69

4.3.1 RDEA to DNA in aqueous environments . . . . . . . . . 71

4.3.2 Implications for atmospheric chemistry . . . . . . . . . . 75 



\section{Introduction}

Liquid water is one of the most fascinating substances on earth. It is omnipresent in the earth's oceans, lakes and rivers, as well as in the billions of cells assembling our bodies. Even more important than the sheer abundance of liquid water are its unique properties, and the surfaces or interfaces that result. These range from the liquid water-air interface on the oceans' surface to interfaces with biological matter in cells. These interfaces in nature are the sites where many of the important chemical and electronic processes take place. This work is dedicated to solvated electrons in liquid water and at liquid water interfaces, to their binding energies, lifetimes and the implications of these physical properties. In a simple picture, a solvated electron in liquid water is just an electron dissolved in water - as we know it from dissolved ions. In the so called 'cavity model', the hydrated electron is on average surrounded by six water molecules which form a non-spherical cage with an average diameter of $4-6 \AA$ [1]. Within this cavity, the electron is stabilized via hydrogen bond interactions with the surrounding water molecules [2].

What is it that makes the hydrated electron such an appealing species so that, since its discovery in 1962 [3], there are on average more than 100 publications per year related to it? The reason is that the hydrated electron plays an important role in many different fields of research in physics, chemistry and biology. Solvated electrons in aqueous solution are formed either through extreme impacts of particles, or by radiation [3-5]. When liquid water - or a species in aqueous solution - is ionized, it involves the ejection of an electron, which is subsequently trapped and solvated by water molecules. The resulting hydrated electron is highly reactive and accordingly plays an important role in many processes initiated by ionizing radiation in aqueous environments. The toxic effect of ionizing radiation on genetic material in cells is connected to cancer formation and therapy and it is therefore a vigorous field of research. Ionizing radiation formally induces two different DNA damaging processes in cells: Direct excitation or ionization of the DNA and the reactive attack by secondary species, which are products from the ionization of neighboring (water) molecules. The important secondary species in this context are $\mathrm{OH}$ radicals and more or less energetic electrons. Both are formed as products of the ionization of water. For a long time it was believed that the $\mathrm{OH}$ radical plays the leading part, while damage induced by secondary electrons was not considered 
to be significant. Only in the last couple of years has the damaging potential of electrons been clearly revealed [6-11]. Today, DNA damage induced by free electrons with kinetic energies between zero and some tens of electron volts is well understood $[6,9]$. The class of these particular electrons is called low kinetic energy electrons. However, the riddle continues on the side of the partially and fully hydrated electrons. In aqueous solution, electrons are rapidly slowed down by collisions and stepwise hydration by water molecules finally results in a hydrated electron. Until now, the damaging potential of partially and fully hydrated electrons has remained difficult to judge since it requires information about the energy scale and the nature of these species. This information was first made accessible only through the experiment presented in this thesis [12].

Another interesting example is from the field of atmospheric chemistry. There seems to be a connection between the creation of the Antarctic ozone hole and the existence of polar stratospheric clouds (PSC). PSCs consist of water ice or nitric acid/ice particles with a major composition of $\mathrm{H}_{2} \mathrm{O}$ and only form in the winter lower polar stratosphere $[13,14]$. A scenario that has been discussed is the splitting of chlorine-containing molecules $\mathrm{RCl}$ - such as chlorofluorocarbons (CFCs), $\mathrm{HCl}$ and $\mathrm{ClONO}_{2}$ - on the surface of these water ice particles. Recent experiments suggest that the splitting of chlorine-containing molecules on the ice surface is initiated by the attachment of a partially solvated surface electron to the molecules [15-18]. These electrons originate from the impact of ionizing cosmic rays on the water ice particles (see Fig. 1.1). In the attachment process, such an electron enters an anti-bonding orbital of the $\mathrm{RCl}$ molecule, which results in the release of a $\mathrm{Cl}^{-}$. Subsequent reaction steps convert the $\mathrm{Cl}^{-}$into $\mathrm{Cl}$ radicals, which destroy ozone via well known reaction pathways [19]. Understanding this attachment phenomenon demands knowledge about the energetics of partially solvated electrons on the surface of water.

The quantity that defines the energy scales of the solvated electron in liquid water is its vertical binding energy (VBE). The VBE is the energy required to remove the electron from its hydration cavity and to completely separate it from the water, while maintaining the initial geometry of the system. For cluster systems, the term vertical detachment energy (VDE) is also common in this context. This quantity would not only allow a promising new insight into radiation induced DNA damage and the ozone depletion mechanism mentioned above, but processes involving electrons in aqueous environments in general. It might, for example, also shed new light on the nature of electron transfer processes in aqueous environments, such as the aqueous tunneling pathways between electron-transfer proteins [20]. Additionally, the knowledge gained could enrich our understanding of the interaction of charged species with water - or with a hydrogen bond network in general - and thus deliver valuable benchmark data for further theoretical investigations. 


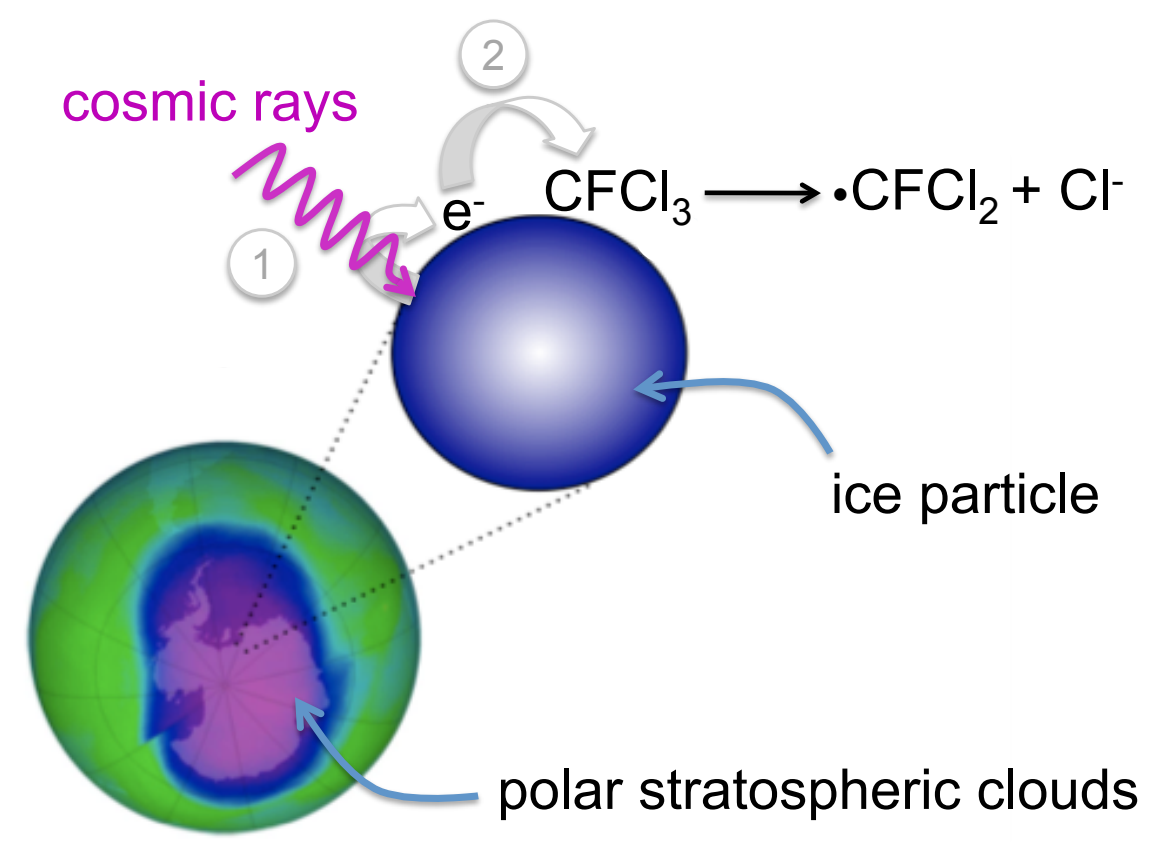

Fig. 1.1: The figure shows the initial steps of a reaction mechanism discussed in the context of the Antarctic ozone hole. (1) Ionizing cosmic rays generate partially solvated electrons on the surface of ice particles. (2) These electrons attach to chlorine-containing molecules (here: $\mathrm{CFCl}_{3}$ ) adsorbed on the ice surface and thereby induce the splitting of a molecular bond.

At this point, it is certainly legitimate to ask for the reasons of the decisive lack of knowledge about the absolute energy scale of the hydrated electron. The answer is quite simple: Directly accessing the vertical binding energies of solvated electrons in liquid water, and in particular of partially hydrated electrons, is a challenging task. It is challenging because it demands the realization of time-resolved photoelectron spectroscopy experiments on a liquid water sample. On the one hand, it requires suitable pulsed laser radiation that allows a time-resolved photoelectron spectroscopy experiment with a time resolution of at least 1 picosecond. On the other hand, it demands a liquid water sample under high vacuum conditions a prerequisite in order to be able to perform photoelectron spectroscopy. These challenges presented an insurmountable obstacle until our group performed the very first time-resolved photoelectron spectroscopy experiments on a liquid water surface in 2007. In our setup, we use liquid micro jet technology in vacuum and a high harmonic light source, which is driven by a femtosecond laser system [21-23]. These experiments were a proof of principle and paved the way for further studies, such as the ones presented in this thesis. 
While the direct investigation of liquids is challenging, experimental and theoretical situations become much easier for finite sized systems, i.e. for anionic water clusters. Several groups investigated anionic water clusters as model systems for bulk hydrated electrons [24-36]. The determined values for the vertical detachment energies (VDE) vary strongly with cluster size and there is clear evidence for the existence of cluster isomers with distinctly different electron solvation motifs. However, the extrapolation of experimental cluster data to yield reliable values for bulk liquid water is difficult and controversial. The current water cluster data provides an interesting starting point in discussing the energetic properties and possible binding motifs of the solvated electron in liquid water and at liquid water interfaces. However, a complete understanding inevitably requires photoelectron spectroscopy measurements, such as those presented in this thesis.

\subsection{Structure and dynamics of the hydrated electron}

Almost 150 years ago, W. Weyl investigated the interaction of ammonia and alkali metals and observed characteristic color changes [37]. About 50 years afterwards, in 1908 C. A. Kraus connected Weyls observations with a new anionic species: the solvated electron [38]. However, it was not until 1962 that the existence of solvated electrons in liquid water was demonstrated by E. Hart and J. W. Boag [3]. They irradiated a water sample and recorded a broad absorption band located at $720 \mathrm{~nm}$ which they attributed to the absorption spectrum of the hydrated electron $[4,5]$. Since then, hydrated electrons have captured the attention of physical scientists, because they represent a particularly important example of electron solvation.

In the absence of electron scavengers, the equilibrated form of the hydrated electron has a microsecond lifetime. The hydrated electron and its solvation cavity have to be considered as a dynamic ensemble in which the configuration of the water molecules constantly changes due to translation, vibration and rotation effects. However, on average the arrangement of water molecules around the electron can be described by a structure in which 6 water molecules octahedrally surround the electron. Each water molecule is pointing an $\mathrm{OH}$ group into the center of the cavity in order to stabilize the electron via hydrogen bond interactions. This structural arrangement is known as the Kevan's structure of the hydrated electron $[1,39]$. Recent experimental and theoretical investigations support this arrangement and additionally allow a detailed insight into the molecular structure and electronic interactions. The ground state of the hydrated electron is an $s$-type orbital which is of spherical symmetry and extends over the first coordination sphere. Recent calculations show that a considerable amount of the electron density is localized in 
the oxygen $2 p$ orbitals of the hydrating $\mathrm{OH}$ groups [2]. The first electronically excited states of the hydrated electron are 3 non-degenerate $p$-type orbitals and the $720 \mathrm{~nm}$ absorption band is attributed to the $s \rightarrow p$ transition [40]. The relaxation dynamics associated with $s \rightarrow p$ excitation of the hydrated electron are of considerable interest and were the subject of many experimental investigations [41-46]. These timeresolved transient absorption spectroscopy experiments revealed that subsequent to electronic excitation, the transient absorption spectrum is shifted much further to the infrared, and recovery of the equilibrium absorption occurs within $1-2$ ps.

Of particular importance for the experiments presented in this thesis are the ultrafast dynamics of electron detachment. This means the mechanisms and relaxation dynamics of electrons ejected from suitable precursors subsequent to photon absorption. The following sections 1.1.1 and 1.1.2 highlight electron detachment for two different cases: (1) two-photon ionization of liquid water, and (2) single photon ionization of the $\left[\mathrm{Fe}(\mathrm{CN})_{6}\right]^{4-}$ complex in aqueous solution. Both are important for the experiments presented in this thesis.

\subsubsection{Two-photon ionization of liquid water}

In liquid water, each ionization event produces a hydroxyl radical, a hydronium ion, and a solvated electron within the first few picoseconds. In the case of a two-photon ionization process this gives:

$$
\mathrm{H}_{2} \mathrm{O}_{(\mathrm{aq})}+2 h \nu \longrightarrow \mathrm{OH}_{(\mathrm{aq})}+\mathrm{H}_{3} \mathrm{O}_{(\mathrm{aq})}^{+}+e_{(\mathrm{aq})}^{-}
$$

An important issue concerning the ionization mechanism in liquid water is how it changes with the excitation energy [47-55]. Insight into the ionization mechanism is obtained via the recombination kinetics of the electron with its geminate partners $\mathrm{OH}$ and $\mathrm{H}_{3} \mathrm{O}^{+}$. The likelihood of geminate recombination strongly depends on the initial separation of the recombination partners, also called electron ejection length. The greater the separation the less likely is geminate recombination. The experimental findings reveal 3 different ionization mechanisms depending on the excitation energy [47]:

(1) Low excitation energies: Photoionization occurs for excitation energies as low as $6.5 \mathrm{eV}[55]$ and proceeds via a proton-coupled electron transfer mechanism [47]. Here, nuclear motion after molecular excitation allows the system to attain a favorable geometry for transferring the excited electron into a localized trap state. These trap states are randomly arising hydration cavities within the fluctuating hydrogen bond network of the liquid, and are also called preexisting traps. Accordingly, 
ionization via this mechanism is only possible if a suitable (preexisting) trap is available in close proximity $(\sim 1 \mathrm{~nm})$ to the ionization event. Importantly, this mechanism completely bypasses the conduction band of the liquid [24].

(2) In case the excitation energy exceeds the adiabatic onset of the conduction band of about $7 \mathrm{eV}$ [24], the proton-coupled electron transfer mechanism competes with autoionization of the molecular excited state. Here, excitation promotes a valence electron into the lowest unoccupied orbital which then couples to the conduction band and ejects the electron into it. At low excitation energies, the rate of autoionization strongly depends on the extent of nuclear and solvent rearrangement which needs to take place in order to transfer the electron into the conduction band. The ratio between the two ionization channels - proton-coupled electron transfer and autoionization - as a function of the excitation energy is not yet completely resolved. However, Laenen et al. investigated the dynamics of the charge separation process of water ionization after excitation with $9 \mathrm{eV}$ and found that the proton-coupled mechanism takes place, exclusively [48].

(3) With excitation energies above $9.5 \mathrm{eV}$, the electron ejection length starts to increase as a function of excitation energy. At an excitation energy of $9.5 \mathrm{eV}$, the electron ejection length amounts to about $1.5 \mathrm{~nm}$, while it is about $4 \mathrm{~nm}$ at $12.4 \mathrm{eV}$ excitation energy [47]. This behavior is due to the fact that higher unoccupied (Rydberg-type) orbitals are increasingly accessible, which makes the autoionization mechanisms increasingly important. In addition, above $9.8-9.9 \mathrm{eV}$ direct optical transitions into the conduction band become accessible [47]. In either case, the final electronic states are very diffuse and extend over many water molecules.

It is important to note that ionization channels also compete with $\mathrm{OH}$ bond dissociation: $\mathrm{H}_{2} \mathrm{O}_{(\mathrm{aq})}+h \nu \rightarrow \mathrm{H}_{(\mathrm{aq})}+\mathrm{OH}_{(\mathrm{aq})}$. Elles et al. investigated the ratio between ionization and $\mathrm{OH}$ bond dissociation for two-photon excitation energies of $8.3,9.3$, and $12.4 \mathrm{eV}$. They found, that the dissociation channel dominates for $8.3 \mathrm{eV}$ excitation, while the ionization channel dominates for the highest excitation energy, and the two decay channels occur with nearly equal probability for $9.3 \mathrm{eV}$ excitation [56].

\subsubsection{Photodetachment from $\left[\mathrm{Fe}(\mathrm{CN})_{6}\right]^{4-}$ in aqueous solution}

The ferrocyanide anion has been a favorite system for photolytic generation of hydrated electrons [43], primarily because of the high electron quantum yield. In water, the $\left[\mathrm{Fe}(\mathrm{CN})_{6}\right]^{4-}$ complex strongly absorbs in the ultraviolet spectral range for $\lambda \leq 313 \mathrm{~nm}$ [57], corresponding to the promotion of an electron into the chargetransfer-to-solvent (CTTS) manifold [58]. This diffuse state rapidly collapses, 
involving electron relaxation/solvation dynamics. The electron photodetachment from the iron-complex has been studied in detail by Lenchenkov et al. [59]. They captured the relaxation of electrons detached from aqueous $\left[\mathrm{Fe}(\mathrm{CN})_{6}\right]^{4-}$ as well as the geminate recombination using femtosecond pump-multicolor probe spectroscopy. For an excitation wavelength of $\lambda=255 \mathrm{~nm}$ they found that the spectral signature of the solvated electron appears within $310 \mathrm{fs}$ after the CTTS excitation and the solvation time amounts to about $570 \mathrm{fs}$. Electrons ejected from the $\left[\mathrm{Fe}(\mathrm{CN})_{6}\right]^{4-}$ complex are completely relaxed within a few ps [59].

Of particular importance for the experiments presented in this thesis is the electron ejection length, which is the initial distance between the detached electron and its parent. Evaluation of the geminate recombination kinetics indicate that the ejected electron starts out on average five solvent shells (about $15 \AA$ ) away from the oxidized iron complex [59]. Interestingly, this result stands in strong contrast to CTTS ejection from $\mathrm{I}^{-}$or $\mathrm{Cl}^{-}$where stabilized electron-radical atom pairs are formed [60]. In addition, Lenchenkov et al. [59] found that the geminate recombination increases with ionic strength. This result can most simply be rationalized by considering the association of $\mathrm{K}^{+}$counter-ions that diminish the Coulomb repulsion between the solvated electron and the ferrocyanide species formed on detachment. In other words, their findings suggest that the majority of the $\left[\mathrm{Fe}(\mathrm{CN})_{6}\right]^{4-}$ complexes in solution are paired with one, two or three $\mathrm{K}^{+}$ions. In one of our experiments we use $267 \mathrm{~nm}$ photons to photodetach electrons from $\left[\mathrm{Fe}(\mathrm{CN})_{6}\right]^{4-}$ complexes in a $0.5 \mathrm{M}$ aqueous solution of potassium ferrocyanide $\mathrm{K}_{4}\left[\mathrm{Fe}(\mathrm{CN})_{6}\right]$. For this concentration, the major species in solution is $\mathrm{K}_{3}\left[\mathrm{Fe}(\mathrm{CN})_{6}\right]^{-}$resulting in a neutral $\mathrm{K}_{3}\left[\mathrm{Fe}(\mathrm{CN})_{6}\right]$ complex after photodetachment [59].

\subsection{Anionic water clusters and electrons at water interfaces}

Anionic water clusters are particularly valuable systems for our understanding of electron hydration. Therefore, they have captured the interest of many scientists, since they were first detected in mass spectrometry experiments in 1981 [61]. Water clusters form in the vacuum expansion of water, and subsequent electron attachment results in anionic water clusters. These clusters usually pass a mass spectrometer unit which allows to select specific cluster sizes for different kinds of experimental investigations: electronic absorption [62], infrared (IR) action spectroscopy [63-69] as well as photoelectron [28,70-72] and time-resolved photoelectron spectroscopy $[25,26,73]$. Various theoretical studies accompanied the experimental investigations and contributed to our understanding of anionic water clusters [31-36, 74-82]. 
One of the central questions of anionic water cluster research ever since is how a water cluster binds an excess electron. This means the binding motif as well as the respective binding energy or vertical detachment energy (VDE). Already in 1988, Barnett et al. [31] theoretically predicted the existence of two different cluster isomers: (1) one with an internally solvated electron in which the electron resides in the center of the cluster and is well solvated by the surrounding water molecules, and (2) an isomer with the electron bound to the cluster surface. Although following experiments confirmed the existence of different isomers, their interpretation has been subject to debate $[26,32,33,83,84]$. Only very recently, after huge experimental and theoretical efforts over almost 30 years, this issue slowly starts to be resolved $[29,30,35,36]$.

Importantly, two cases have to be distinguished: In the first case, the water clusters are warm and possess enough internal energy to allow for significant structural rearrangement subsequent to electron attachment. The second case are cold clusters in which reorientation is kinetically inhibited and solvation is only possible to some extent. The latter case is comparable to electron solvation phenomena on the surface of ice [85-89]. There, electrons initially localize at pre-existing defects and subsequently induce dynamic reorientations of adjacent water molecules. Such a structural evolution is thermally activated and hence accelerated at increased temperatures. Electrons attached to cold water clusters or ice surfaces will thus localize on the surface, resulting in more or less diffuse surface solvated electrons with lifetimes up to minutes [36,89]. Fig. 1.2 a) depicts this situation for an electron attached to a cold $(50 \mathrm{~K})$ cluster of 32 water molecules. The relaxation process is not completed and the electron is trapped in a metastable surface state. The pictures in Fig. 1.2 are from ab inito molecular dynamics simulations by Ondrej Marsalek [36].

In warm clusters or on liquid water surfaces, the situation is distinctly different, since the system is able to attain the energetically most favorable situation. For liquid water it is believed that a bulk electron, which is an internally solvated electron, represents the energy minimum. In mixed-quantum classical molecular dynamics simulations of excess electron relaxation dynamics at water/air interfaces Madarasz et al. found that electrons initially located on a liquid water surface at $300 \mathrm{~K}$ dive into the bulk within a couple of picoseconds [85]. For clusters this brings up the interesting question about how a water cluster $\left(\mathrm{H}_{2} \mathrm{O}\right)_{n}$ of particular size $\mathrm{n}$ prefers to bind an excess electron. Recent experiments suggest that small anionic clusters up to a size of about $n=25-30$ carry their electron on the surface, exclusively $[29,30]$. An internal solvation is energetically unfavorable, since it goes along with the loss of hydrogen bond interactions between the water molecules. However, for cluster sizes $n \geq 50$ the situation is reversed and the electron primarily 
a)

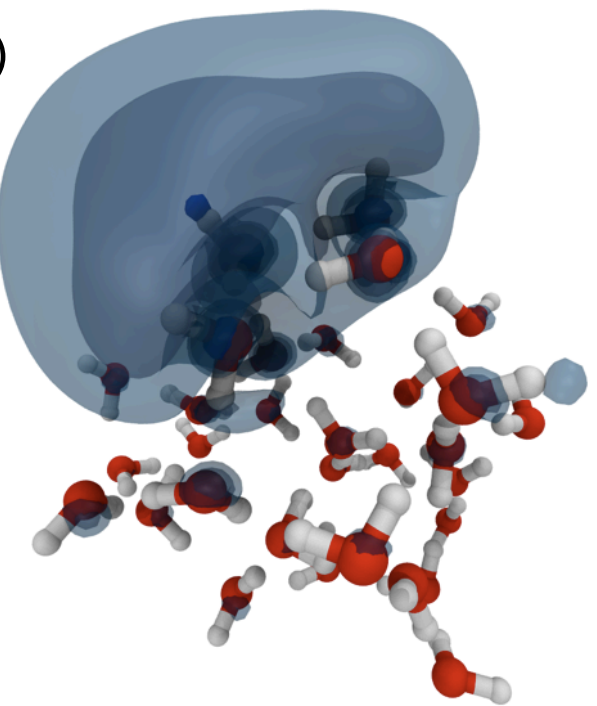

b)

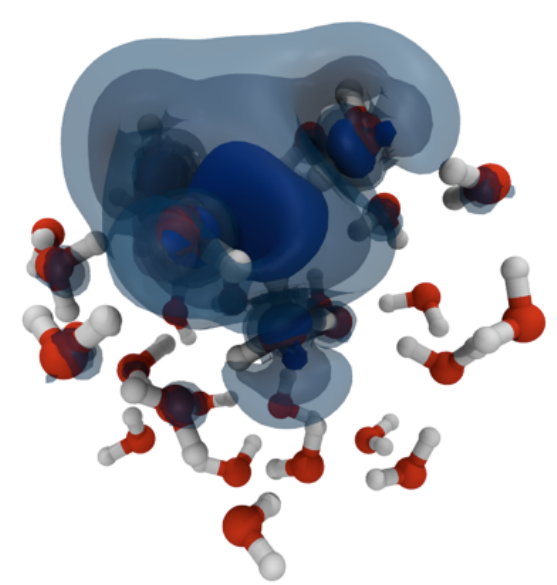

Fig. 1.2: a) Electron attached to a cold $(50 \mathrm{~K})$ cluster of 32 water molecules. The relaxation process is not completed and the electron is trapped in a metastable surface state. b) Equilibrated structure of an electron in a warm (300 K) cluster of 32 water molecules. The pictures are snapshots from ab inito molecular dynamics simulations by Ondrej Marsalek [36].

occupies internal states [30]. Fig. 1.2 b) depicts the equilibrated structure of an electron in a warm $(300 \mathrm{~K})$ cluster of 32 water molecules. The pictures is a snapshot from an ab inito molecular dynamics simulation by Ondrej Marsalek [36].

The Johnson group was able to identify the binding motif associated with surface solvated electrons using infrared (IR) action spectroscopy [63-69]. In the spectral region of the bending transitions of water, they found a strong peak which is significantly red-shifted from the $\mathrm{H}_{2} \mathrm{O}$ bend fundamental. In combination with electronic structure calculations, they suggest that this feature is from a doubleacceptor (AA) water molecule with both $\mathrm{H}$ atoms pointing into the diffuse orbital of the excess electron [63]. Further information about the structure of water cluster anions comes from recent electronic structure calculations by Jordan [74-76], Head-Gordon [77-79], and Khan [80-82].

For anionic water clusters, the vertical detachment energies (VDEs) of internally solvated electrons are constantly higher than the VDEs of surface solvated electrons $[29,30]$, and both VDEs are steadily increasing with cluster size. Although VDEs are available for a broad range of cluster sizes, it is not trivial to extrapolate them to the bulk. The major problem is that they do not scale fully linearly 
with $\mathrm{n}^{-1 / 3}$, which corresponds to the inverse cluster radius. Anionic water clusters allow valuable insights into electron solvation phenomena in aqueous environments. However, it is questionable if extrapolations from cluster data can provide reliable data for bulk liquid water and its surface.

\subsection{Electron induced strand breaks in DNA}

Deoxyribonucleic acid (DNA) contains the genetic instructions used in the development and functioning of all known living organisms and some viruses. In most living organisms, the DNA is organized in long structures called chromosomes which are stored inside the cell nucleus. Organelles, such as mitochondria or chloroplasts have additional circular DNA strands referred to as plasmids. DNA is a remarkably stable molecule and even if it happens to be damaged, a cell has several mechanisms to repair it. However, not all damages can be repaired and are consequently passed down to the two daughter cells when the cell divides. Accordingly, with increasing age of the organism, the DNA contains more and more damaged parts. While most of them are harmless, DNA damage in sections containing information for cell replication or lifetime can result in dangerous cancer cells or cell death. Accordingly, understanding mechanisms of DNA damage is of significant importance for both cancer prevention and therapy.

Ionizing radiation can damage DNA in several ways: (1) via direct excitation or ionization of the DNA or (2) the reactive attack by secondary species generated in an initial ionization event of the DNA itself or surrounding (water) molecules. Since the DNA system has very efficient deexcitation mechanisms and also the ability to refill electron holes [90], DNA damage primarily occurs through the second scenario. Ionization of the DNA results in radical DNA cations and electrons. Ionization of water leads to electrons and $\mathrm{OH}$ radicals, which can then attack DNA and break chemical bonds. For a long time it was believed that the $\mathrm{OH}$ radical plays the leading part, while damage induced by secondary electrons was not considered as being significant. It was not until the last couple of years that the damaging potential of electrons was clearly revealed [6-11].

When analyzing the experiments and theoretical investigations dedicated to electron induced DNA damage one has to distinguish two cases: (1) electron attachment to dry DNA samples or DNA molecules in the gas phase and (2) to DNA in its natural surrounding, which means in aqueous solution. 


\section{Electron attachment to dry DNA}

In 2000 novel experimental findings from the Sanche group initiated the discussion on electron induced strand breaks in DNA [6]. They irradiated dry DNA samples, containing only structural water molecules, with an electron beam of known kinetic energy in the range between $3-20 \mathrm{eV}$ and subsequently analyzed the amount of DNA damage, that is of single- and double-strand breaks. Importantly, they found that not only electrons with kinetic energies above the ionization threshold of DNA of about 7.5 to $10 \mathrm{eV}$ induce strand breaks, but also electrons with kinetic energies as low as $3 \mathrm{eV}$. Following experimental and theoretical investigations revealed that the strand breaks are initiated by electrons attaching to the $\pi^{*}$ orbitals of the DNA bases to form so-called core-excited resonance states. Here, the electrons are captured by an electronically excited state and lose energy by simultaneously exciting another electron from a $\pi$ to a $\pi^{*}$ orbital: $e^{-}+\pi^{2} \rightarrow \pi^{1} \pi^{* 2}[9]$.

Further experimental and theoretical investigations additionally revealed another electron attachment scenario resulting in so-called shape-resonance anions [9,91,92]. In contrast to the core-excited resonances in which an electron attaches and excites another electron, in a shape-resonance, an electron attaches to a $\pi^{*}$ orbital of a DNA base without further electronic excitation. Accordingly, shape-resonance states lie considerably lower and can be accessed by electrons with kinetic energies as low as $0.1-2 \mathrm{eV}$. Subsequently to electron attachment to the $\pi^{*}$ a through bond electron transfer into the sugar-phosphate $\mathrm{C}-\mathrm{O} \sigma^{*}$ can occur which subsequently fragments into a carbon radical and a very stable phosphate-site anion [9]. Similar to electron attachment to the DNA base $\pi^{*}$ orbital, electrons with kinetic energies $>2 \mathrm{eV}$ can resonantly attach to the $\mathrm{P}=\mathrm{O} \pi^{*}$. However, in both cases it is important to note that the majority of the shape-resonance anions undergo autodetachment and are thus non-reactive [9].

Today, DNA damage in dry DNA samples induced by electrons with kinetic energies between about zero and some tens of electron volts is well understood $[6,9,10]$. Unfortunately, this situation cannot simply be transferred to DNA in its natural surrounding, that is DNA solvated by water.

\section{Electron attachment to DNA in aqueous environments}

An aqueous environment significantly influences the energetics of DNA (sub)units [9, 10,93-98] and herewith also the nature of electron attachment. Experimental evidence comes from photoelectron spectroscopy experiments on anionic DNA bases in the gas phase and in small water clusters. These experiments clearly show a transition from dipole-bound electrons in the gas phase to covalently 
bound electrons in microsolvated DNA bases (DNAbase) $)^{-} \cdot\left(\mathrm{H}_{2} \mathrm{O}\right)_{\mathrm{n}}[99,100]$. In addition, vertical electron detachment energies (VDEs) of the anionic DNA bases (DNAbase $)^{-} \cdot\left(\mathrm{H}_{2} \mathrm{O}\right)_{\mathrm{n}}$ steadily increase with increasing number of solvating water molecules. Theoretical investigations confirm dramatic changes in the energies of neutral and anionic DNA species upon solvation [9, 10,93-98].

Besides, in radiation chemistry and biology, the role of water in DNA damage induced by ionizing radiation is not yet completely revealed. On the one hand, the amount of DNA strand breaks by irradiation with $\gamma$-rays in aqueous solution is 3 orders of magnitude higher compared to the gas phase [101]. However, this significant difference can only partially be attributed to the damaging potential of $\mathrm{OH}$ and (completely) hydrated electrons [8]. The situation is further complicated by the fact that initially free electrons in aqueous solution are rapidly slowed down by collisions and stepwise hydrated by water molecules, finally resulting in hydrated electrons. In this context, Wang et al. recently found experimental evidence that so called pre-hydrated electrons, which is a class of electrons between free low energy electrons and completely hydrated electrons, may play an important role $[7,8,102]$.

Taken together, the current situation on electron induced DNA damage in aqueous environments resembles a jigsaw puzzle. For the first time, our experiments reveal the absolute energy scale and the nature of partially and fully solvated electrons in water [12] - an essential piece of knowledge in this context. With this information we attempt to combine the different jigsaw pieces to a simple and conclusive picture which allows to understand electron attachment to DNA in aqueous environments (see section 4.3.1).

\subsection{Cosmic ray driven electron induced ozone depletion}

Every year, in the springtime, the ozone hole forms over the Antarctic region (and to a smaller extent also in the arctic region) in the lower stratosphere at about $18 \mathrm{~km}$ over the pole. The photochemical model for ozone depletion proposes a three step mechanism to explain this observation [14,17]: (1) In the first step, the photolysis of chlorofluorocarbons (CFCs) produces $\mathrm{Cl}$ in the tropical upper stratosphere at altitudes of about $40 \mathrm{~km}$ as proposed by Molina and Rowland [19]. Further reactions with atmospheric molecules, such as $\mathrm{CH}_{4}$, ozone $\mathrm{O}_{3}$ and $\mathrm{NO}_{2}$ results in the inorganic chlorine-containing species $\mathrm{HCl}$ and $\mathrm{ClONO}_{2}$. (2) Air circulation then transports these species from the tropical upper stratosphere to the lower polar stratosphere at an altitude of $15-20 \mathrm{~km}$. (3) There, heterogeneous chemical reactions on the surface of polar stratospheric clouds (PSCs) convert the inactive chlorine-containing compounds into the photo-reactive species $\mathrm{Cl}_{2}$ : 


$$
\mathrm{HCl}+\mathrm{ClONO}_{2} \longrightarrow \mathrm{Cl}_{2}+\mathrm{HNO}_{3} .
$$

PSCs consist of water ice or nitric acid/ice particles with a major composition of $\mathrm{H}_{2} \mathrm{O}$ and only form in the winter lower polar stratosphere at temperatures of about $150-200 \mathrm{~K}$ and at altitudes between $15-25 \mathrm{~km} \mathrm{[13].} \mathrm{The} \mathrm{above} \mathrm{reaction}$ takes place on PSCs during the winter time, while the returning sunlight in spring melts the PSCs, releases $\mathrm{Cl}_{2}$ and photolyses it into $\mathrm{Cl}$ radicals which destroy ozone via the $(\mathrm{Cl}, \mathrm{ClO})$ reaction chain [19]. Taken together, this scenario is the common explanation for the Antarctic springtime ozone hole.

However, in the last couple of years it was found that the photochemical model presented above is not consistent with recent observations of the atmosphere's composition and air streams [17]. In addition, an apparent correlation between ozone concentration over the poles and cosmic ray intensity was revealed. Based on this observation and laboratory measurements on the photodissociation of CFCs adsorbed on water ice surfaces, another mechanism for Antarctic ozone hole formation was proposed: The cosmic ray driven electron reaction model for ozone depletion [15-18, 103, 104]. Here, cosmic rays consisting of protons (90\%) and alpha-particles $(9 \%)$ and originating from deep space, generate surface trapped electrons (see section 1.2) on PSCs in the Antarctic winter. These electrons then attach to molecules adsorbed on the surface of the ice particles, such as CFCs, $\mathrm{ClONO}_{2}$, and $\mathrm{HCl}$ which are abundant in the lower polar stratosphere at the beginning of the winter period:

$$
\begin{gathered}
\mathrm{e}_{\text {surface }}^{-}+\mathrm{RCl} \longrightarrow \mathrm{RCl}^{*-} \longrightarrow \mathrm{Cl}^{-}+\mathrm{R} \\
\left(\mathrm{RCl}=\mathrm{CFCs}, \mathrm{ClONO}_{2}, \mathrm{HCl}\right)
\end{gathered}
$$

As the equation shows, electron attachment results in the release of a $\mathrm{Cl}^{-}$via an intermediate anion species $\left(\mathrm{RCl}^{*-}\right)$. The $\mathrm{Cl}^{-}$can subsequently be converted to $\mathrm{Cl}$ atoms to destroy $\mathrm{O}_{3}$ molecules or react with other stratospheric species to form photo-active $\mathrm{Cl}_{2}, \mathrm{ClNO}_{2}$ or $\mathrm{OClO}$ [17]. Interestingly, the cross section for the dissociative attachment of surface solvated electrons to $\mathrm{CF}_{2} \mathrm{Cl}_{2}$ adsorbed on water ice amounts to $1 \cdot 10^{-14} \mathrm{~cm}^{2}$ which is six orders of magnitude higher than the photodissociation cross section $\left(10^{-20} \mathrm{~cm}^{2}\right)$ of $\mathrm{CF}_{2} \mathrm{Cl}_{2}$ [103]. Similar trends can also be expected for other chlorine-containing molecules.

The importance of the cosmic ray driven electron model for ozone depletion is still subject to debate $[17,105,106]$. However, it is consistent with several data collected in the polar atmosphere like the spatial and time correlation between cosmic ray flux and ozone depletion, and the strong decrease of $\mathrm{CFCs}, \mathrm{ClONO}_{2}$, and $\mathrm{HCl}$ by 
the end of the winter period [17]. These are strong arguments that the cosmic ray driven mechanism presented above plays a role in the conversion of halogenated molecules into reactive halogens in the winter lower polar stratosphere and is thus important in the context of ozone hole formation.

A complete picture of the initiating electron attachment step remains elusive, until the binding energy of surface solvated electrons is available. The experimental observations however suggest that the binding energy lies in the range of the anion resonance of halogenated molecules, that is between 1 and $1.5 \mathrm{eV}$ [17]. Obviously, this quantity is crucial for understanding the high efficiency of dissociative electron attachment to chlorine-containing molecules on the surface of ice and presumably many other reactions involving partially solvated electrons. 


\section{Experimental approach}

Our experimental setup represents a unique combination of a high harmonic light source together with liquid micro jet technology in vacuum. The key feature to our experimental approach is generating solvated electrons in the liquid micro jet by a short laser pulse of $267 \mathrm{~nm}$ light and recording photoelectron spectra using a time-delayed $38.7 \mathrm{eV}(32 \mathrm{~nm})$ high harmonic probe pulse (see Fig. 2.1). The experimental setup is schematically depicted in Fig. 2.2. We amplify the output of a femtosecond titanium-sapphire laser before splitting the light into pump and probe pulse. The pump pulse is frequency tripled to $267 \mathrm{~nm}$ UV light and focused onto the liquid micro jet to generate the solvated electrons therein. We generate the high harmonic probe pulse by focusing the $800 \mathrm{~nm}$ light into a capillary perfused by argon gas. There, the high harmonic generation takes place, which results in high-energetic photons exhibiting odd multiples of the fundamental frequency. A toroidal grating selects the $25^{\text {th }}$ harmonic $(38.7 \mathrm{eV}, 32 \mathrm{~nm})$ and focuses it onto the liquid jet. The probe pulse generates photoelectrons, which we analyze and detect with a time-of-flight photoelectron spectrometer. In our experiment, the pump and probe pulses are overlapped nearly collinear in space and their polarization vectors are parallel.

This chapter is dedicated to the details of our experimental setup. Starting with the femtosecond laser system and the amplification unit as well as the generation of the $267 \mathrm{~nm}$ pump pulse in section 2.1. The following section 2.2 is composed of theoretical background information on high harmonic radiation and details on the experimental implementation in our setup. Section 2.3 is dedicated to liquid phase photoelectron spectroscopy. The section includes details on the liquid micro jet technology, which is a prerequisite for this kind of liquid phase studies, and briefly discusses observed liquid phase phenomena and their interpretation. Time-resolved liquid phase photoelectron spectroscopy experiments are shortly addressed and the surface sensitivity of photoelectron spectroscopy is discussed in the context of our experimental conditions. The last part describes our photoelectron analyzer and detection unit, as well as the calibration procedure. 


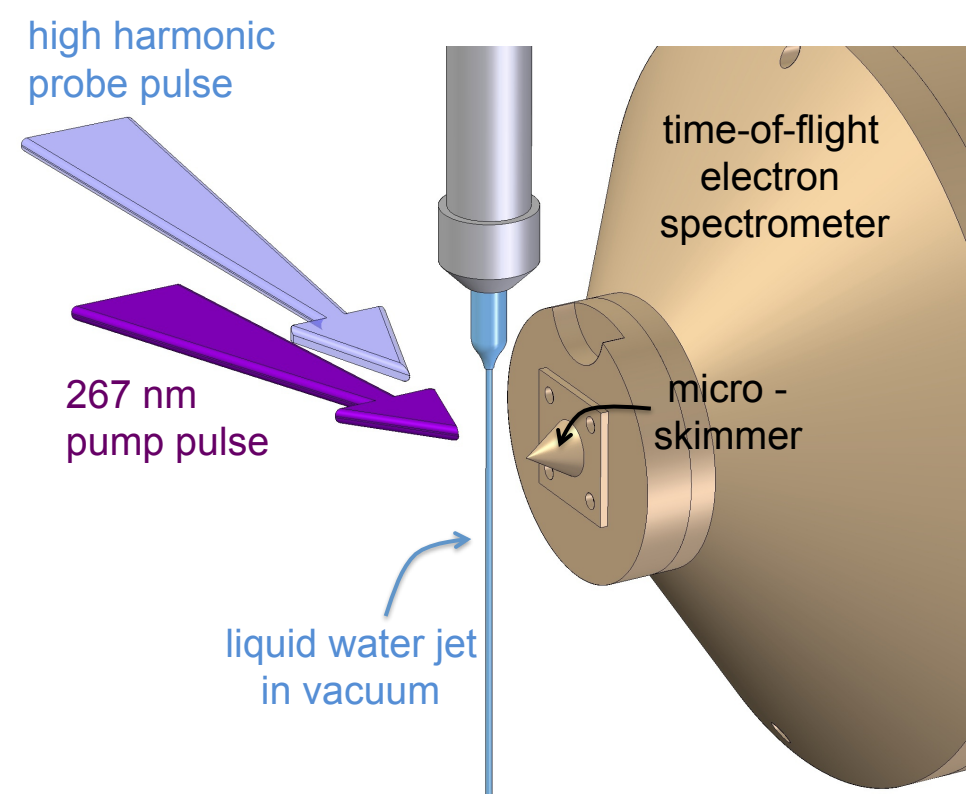

Fig. 2.1: The key feature to our experimental approach is generating solvated electrons in the liquid micro jet by a short laser pulse of $267 \mathrm{~nm}$ light and recording photoelectron spectra using a time-delayed $38.7 \mathrm{eV}(32 \mathrm{~nm})$ high harmonic probe pulse.

\subsection{Laser system}

We use the commercial titanium sapphire laser system Hurricane (Spectra Physics) as a source of $800 \mathrm{~nm}$ femtosecond laser pulses. The system regularly delivers $90 \mathrm{fs}$ pulses with a pulse energy of $1.0 \mathrm{~mJ}$ and a repetition rate of $1 \mathrm{kHz}$ at a central wavelength of $800 \mathrm{~nm}$. However, in the present experiments we use the uncompressed output $(1.45 \mathrm{~mJ}, 800 \mathrm{~nm}, 1 \mathrm{kHz})$, guide it through an additional amplification unit and a grating compressor to obtain $2.1 \mathrm{~mJ} /$ pulse $(100 \mathrm{fs}$ pulse duration, $800 \mathrm{~nm}$ central wavelength, $1 \mathrm{kHz}$ repetition rate). A beam splitter reflects about two thirds of this energy $(1.4 \mathrm{~mJ})$ for the generation of the high harmonic probe pulse. The transmitted radiation $(0.7 \mathrm{~mJ})$ is frequency tripled in two successive $\beta$-barium borate (BBO) crystals to yield up to $40 \mu \mathrm{J}$ of $267 \mathrm{~nm}$ pump light.

The titanium sapphire laser system Hurricane is based on the chirped pulse amplification (CPA) method and consists of four main components: oscillator, pulse stretcher, regenerative amplification unit and pulse compressor. Detailed 


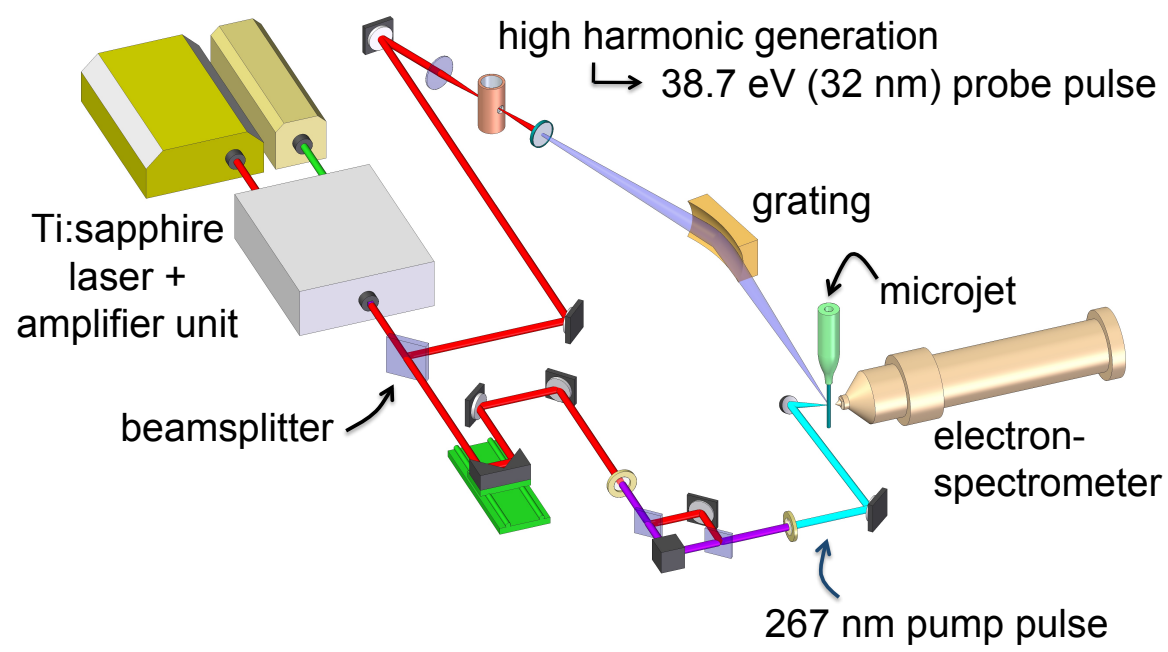

Fig. 2.2: Schematic representation of our experimental setup.

descriptions can be found in references [21,107]. The oscillator (Mai Tai) contains a titanium sapphire crystal inside a resonator. To generate femtosecond laser pulses, a doubled Nd:YAG laser with a central wavelength of $532 \mathrm{~nm}$ pumps the crystal. The emission spectrum of the Ti:sapphire crystal is relatively broad with a maximum at $800 \mathrm{~nm}$. A short disturbance of the resonator geometry induces the mode locking of multiple resonator modes, which results in ultrashort laser pulses of about $50 \mathrm{fs}$. The pulses leave the resonator and pass a pulse stretching unit consisting of a grating and multiple mirrors. The resulting pulse length is in the order of a few picoseconds, which is necessary in order to avoid damage of optical elements in the regenerative amplifier. The central element of the regenerative amplifier unit is another Ti:sapphire crystal inside a resonator. A pulsed Nd:YAG laser (Evolution, 8W, $527 \mathrm{~nm}, 100 \mathrm{~ns}$ ) pumps this crystal at a repetition rate of $1 \mathrm{kHz}$ and induces a population inversion. Time delayed to that, a Pockels cell couples a stretched pulse into the resonator, which is amplified by passing the crystal and inducing stimulated emission therein. After about 20 resonator cycles, another Pockels cell releases the pulse from the resonator. Usually, the pulse then passes a compressor unit (inversely to the stretcher unit) and the final output would be a $90 \mathrm{fs}$ short laser pulse with a pulse energy of $1.0 \mathrm{~mJ}(1 \mathrm{kHz}$ repetition rate, central wavelength of $800 \mathrm{~nm}$ ). However, for the present experiments, we use the output from the regenerative amplifier $(1.45 \mathrm{~mJ})$ and guide it into another, external amplification unit.

This external amplifier is a home-built 3-pass amplifier [21]. The central piece is another Ti:sapphire crystal which is cooled to $18^{\circ} \mathrm{C}$ and pumped by a Nd:YAG 


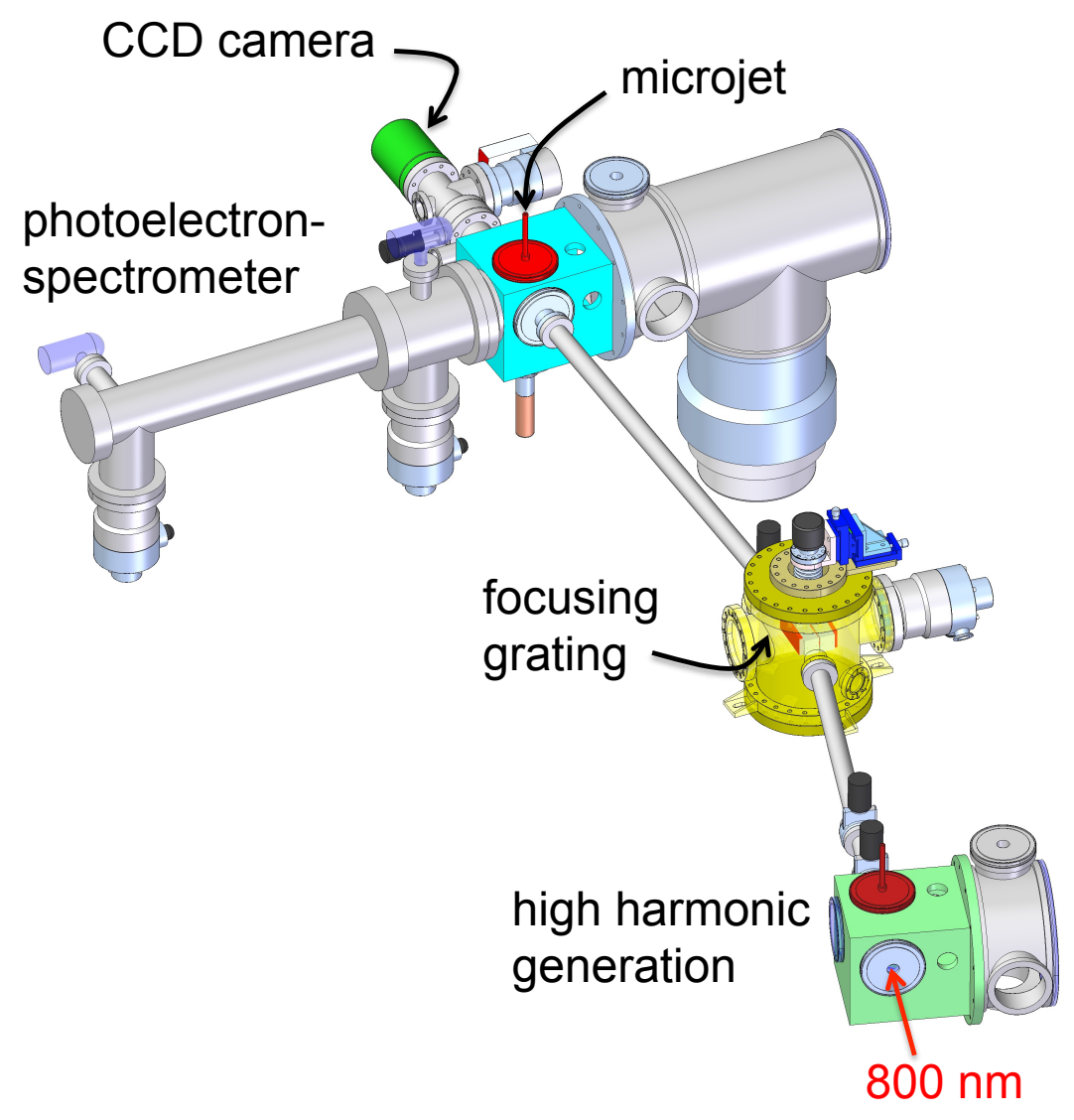

Fig. 2.3: Detailed picture of the vacuum setup composed of our table top beamline (high harmonic generation chamber and grating chamber), the main chamber containing the liquid microjet sample and a time-of-flight photoelectron spectrometer.

laser $(532 \mathrm{~nm}, 1 \mathrm{kHz}, 8 \mathrm{~W},>200 \mathrm{~ns}$, Clark-MXR, ORC-1000). Every pulse from the Hurricane system passes the crystal three times and is amplified to $3.2 \mathrm{~mJ}$. We use a delay generator (Stanford Research Systems) to match the arrival times of the two pulses in the crystal. Subsequent pulse compression takes place in a home-built grating compressor consisting of two gratings (2000 grooves $/ \mathrm{mm}$, Spectrogon), several mirrors and a telescope. The resulting pulses exhibit a pulse energy of $2.1 \mathrm{~mJ}$ and a pulse length of about $100 \mathrm{fs}$.

A beam splitter reflects about two thirds of this energy $(1.4 \mathrm{~mJ} / \mathrm{pulse})$ for the generation of the high harmonic probe pulse. We guide the transmitted radiation $(0.7 \mathrm{~mJ} / \mathrm{pulse})$ over a translation stage, which allows to set the time delay between pump and probe pulse, and subsequently into a UV unit to generate the $267 \mathrm{~nm}$ 


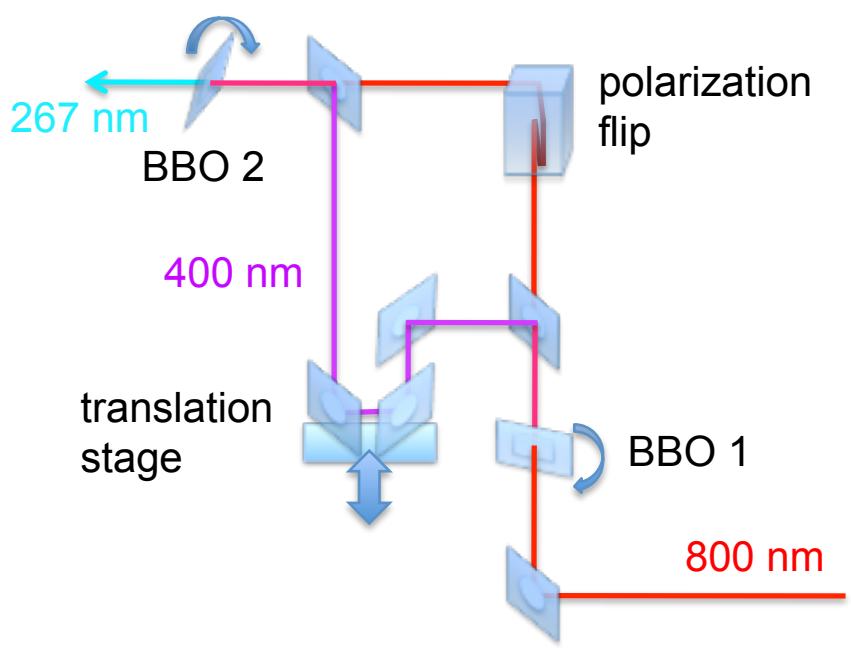

Fig. 2.4: Schematic representation of the UV unit for the generation of the $267 \mathrm{~nm}$ pump pulse via frequency tripling of the $800 \mathrm{~nm}$ fundamental.

pump pulse via frequency tripling of the $800 \mathrm{~nm}$ fundamental (Fig. 2.4). In a first $\beta$-barium borate $(\mathrm{BBO})$ crystal $\left(5 \times 5 \times 1 \mathrm{~mm}^{3}\right)$ we generate the second harmonic of the fundamental and obtain about $170 \mu \mathrm{J} /$ pulse of $400 \mathrm{~nm}$ light. A high reflective mirror for $400 \mathrm{~nm}$ separates the second harmonic from the residual fundamental light. We flip the polarization direction of the residual $800 \mathrm{~nm}$ light from p- to s-polarization and overlap it with the $400 \mathrm{~nm}$ light (s-polarization) in a second BBO crystal $\left(5 \times 5 \times 1 \mathrm{~mm}^{3}\right)$. The temporal overlap of the two pulses in BBO 2 can be adjusted with a small translation stage. With this setup we obtain up to $40 \mu \mathrm{J} /$ pulse of $267 \mathrm{~nm}$ UV light. We either use a quartz prism or high reflective $267 \mathrm{~nm}$ mirrors and a diaphragm to separate residual $400 \mathrm{~nm}$ and $800 \mathrm{~nm}$ light. In the experimental chamber, a parabolic mirror weakly focuses the UV pump pulse onto the liquid jet sample. We control the pump-pulse intensities in the focus between $5-50 \mathrm{GW} / \mathrm{cm}^{2}$ to match the conditions required for the present experiment.

\subsection{High harmonic radiation}

With the advent of high harmonic light sources, ultrashort light pulses in the extreme ultraviolet (EUV) and soft X-ray spectral range were becoming available. This means photons with energies up to $1000 \mathrm{eV}$ delivered in light pulses as short as 100 attoseconds. This kind of radiation opened the door for novel time-resolved studies, which allow fundamentally new insights into dynamical processes on femtosecond (fs) and attosecond (as) timescales [108, 109]. 


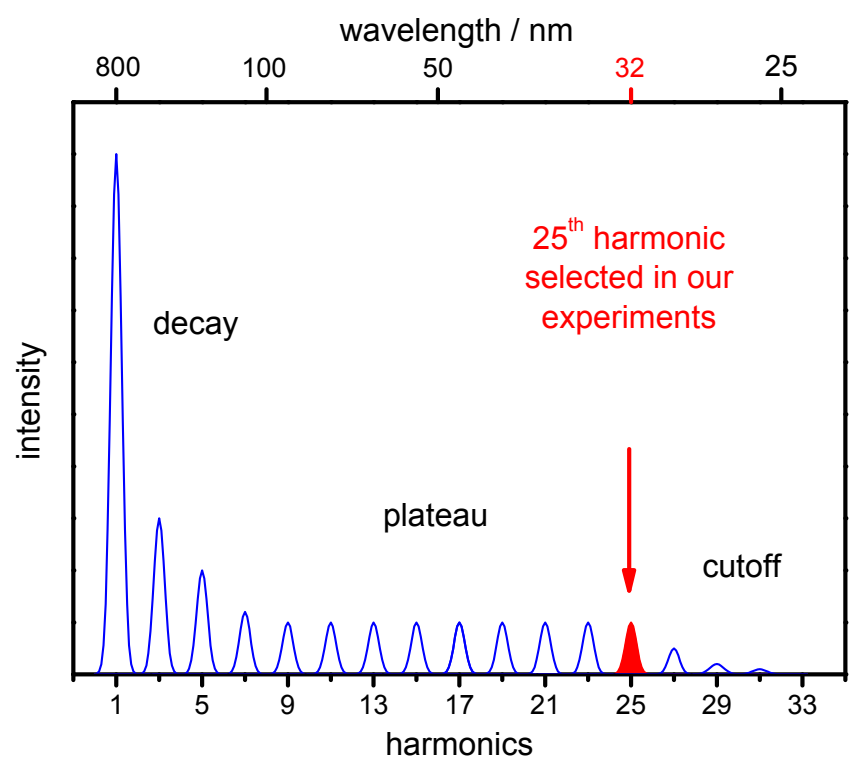

Fig. 2.5: Schematic spectrum of the high harmonics generated in our experiment. We focus the $800 \mathrm{~nm}$ fundamental laser pulses into a capillary filled with argon gas. In our experiments we select the $25^{\text {th }}$ harmonic of the fundamental, which corresponds to a photon energy of $38.7 \mathrm{eV}$ or a wavelength of $32 \mathrm{~nm}$

The key feature to high harmonic generation (HHG) is focusing a short laser pulse into a non linear optical medium, typically a rare gas [110]. When the intense laser field interacts with the medium, different strong field effects take place. One of these effects is the high harmonic generation, which results in high-energetic photons exhibiting multiples of the fundamental photon energy. These multiples also called harmonics - are distributed over a broad spectral region, ranging from the fundamental wavelength in the visible to the extreme UV or even the soft X-ray domain. A schematic spectrum is shown in Fig. 2.5. It consists of discrete, odd harmonics of the fundamental frequency. The fact that only the odd harmonics are generated, is associated with the inversion symmetry of the rare gas medium. The harmonic spectrum displays different domains. First, a strong decrease in intensity within the first harmonics is observed. This decay precedes a plateau region with harmonics of similar intensity. At the end of the plateau, the intensity rapidly drops in the cutoff region.

A prerequisite for HHG is that the interaction of the laser field with the valence electrons of the rare gas medium is on the same order of magnitude, as the atomic Coulomb potential which the valence electrons experience. This means that the amplitude of the laser field is comparable to the atomic Coulomb field. In contrast, 
interaction with a weaker laser field only results in a disturbance of the atomic electronic systems and eventually in the promotion of electrons to electronically excited states. If the laser field is too strong, the atomic systems undergo partial or complete ionization.

High harmonic radiation has a number of appreciable properties. The harmonics are collinear with the driving laser field and exhibit a high degree of spatial coherence. The emission of the harmonic radiation from the gas target occurs in a beam of low divergence. In addition, the high harmonic light pulses are temporally shorter than the fundamental laser pulse. This is due to the fact that high harmonic generation only takes place, when the amplitude of the driving laser pulse exceeds a certain threshold. Accordingly, using phase stabilized and transform limited fundamental light pulses with a pulse duration of a few femtoseconds, allows to generate attosecond high harmonic light pulses [111].

\subsubsection{Semiclassical three-step model}

This section describes high harmonic generation within a semiclassical approach. The model contains the three steps illustrated in Fig. 2.6 and was introduced by Corkum in 1993 [112]. In part a) of Fig. 2.6, the Coulomb potential V $(x)$ of a valence electron is influenced by the electrical field $\mathrm{E}\left(x, t_{0}\right)$ of the electromagnetic light wave. The superposition of both potentials results in a potential surface $\mathrm{V}(x)$ - exE $\left(x, t_{0}\right)$ which is bent down. With a certain, but rather low probability, the electron can tunnel out of the bent potential and enter the continuum. Within the three-step model, an electron which left its parent atom via tunnel ionization is considered as a 'free' electron which is not influenced by the atomic Coulombic field any more. The motion of the electron in the electrical field of the laser light is then described with classical mechanics. In this approach it is assumed that right after leaving the atom, the electron possesses zero kinetic energy $v_{0}=0$.

The tunnel ionization happens at a certain time $t_{0}$ and thus at a certain phase $\phi_{0}=\omega t_{0}$ of the electric field (see bottom of Fig. 2.6). Therefore, two cases have to be distinguished. In the first case, $t_{0}$ lies between a zero-crossing and a subsequent maximum of the electric field: $0^{\circ}<\phi_{0}<90^{\circ}$ or generally $n \pi<\phi_{0}<\left(n+\frac{1}{2}\right) \pi$ $(n=0,1,2, \ldots)$. In this case, the electron is accelerated away from its parent and never returns. The reason for this becomes clear while considering the second case: $90^{\circ}<\phi_{0}<180^{\circ}$ or generally $\left(n-\frac{1}{2}\right) \pi<\phi_{0}<n \pi$. Here, the electric field first accelerates the electron away from its parent atom until the field changes its sign at the zero-crossing $\phi=180^{\circ}$ (or $\phi=n \pi$ ). Subsequently, the electron is decelerated until it formally possesses zero kinetic energy at a time $t_{u}$ or a phase $\phi_{u}=360^{\circ}-\phi_{0}$. Accordingly, the area under the curve on the bottom of Fig. $2.6 \mathrm{~b}$ ) between $t_{0}$ 
and the subsequent zero-crossing equals the one between this zero-crossing and $t_{u}$. Past $t_{u}$, the electric field accelerates the electron back to its parent where it eventually recombines at at time $t_{1}$ by releasing a high harmonic photon (part c) of Fig. 2.6). It is obvious that linear polarized laser light is a prerequisite for a successful recombination.

The recombination competes with other possible scenarios. One of them is elastic scattering connected to above threshold ionization (ATI) [113]. The other is inelastic scattering with the electrons of the parent atom. In this case, a second electron is liberated from the system resulting in a doubly ionized species. This process is called non-sequential double ionization (NSDI) [114].

In the case of a successful recombination of the electron with its parent atom, the energy which is released as a high harmonic photon can be calculated with classical mechanics. It is the sum of the electrons kinetic energy $E_{\text {kin }}\left(t_{1}\right)$ at the time of recombination and the ionization potential $\mathrm{I}_{p}$ of the atom. At the time $t_{1}$, which depends on $t_{0}$, the electron possesses a kinetic energy between 0 and $3.17 \mathrm{U}_{p}$. The ponderomotive potential $\mathrm{U}_{p}$ is the average kinetic energy of the electron in the laser field:

a)
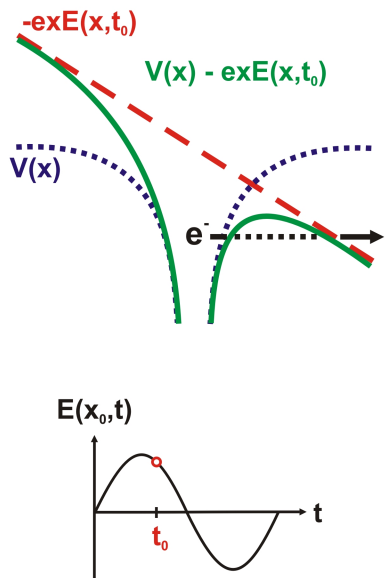

b)
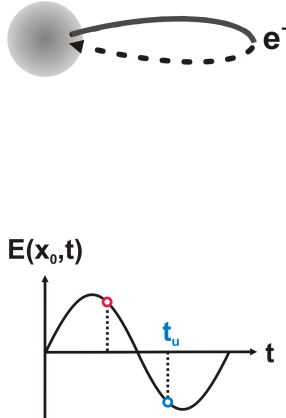

c)
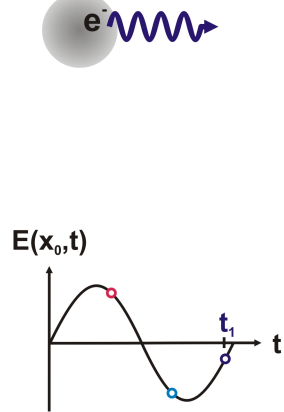

Fig. 2.6: Illustration of the semiclassical three-step model for high harmonic generation. a) An electron leaves the system via tunnel ionization at a certain time $t_{0}$. b) It is accelerated by the electric field of the laser light and c) eventually returns to its parent atom and recombines at a time $t_{1}$ by releasing a high harmonic photon. 


$$
\mathrm{U}_{p}=\frac{e^{2} \mathrm{E}_{L}^{2}}{m_{e} \omega_{0}^{2}}=\text { const } \mathrm{I}_{L} \lambda_{0}^{2}
$$

Here, $e$ designates the elementary charge and $m_{e}$ is the rest mass of the electron. $\mathrm{E}_{L}$ is the amplitude of the electric field of the laser light, $\mathrm{I}_{L}$ describes its intensity, $\lambda_{0}$ is the central wavelength and $\omega_{0}$ the respective angular frequency. The maximum energy of the high harmonic photons and thus the experimentally observed cutoff energy in the high harmonic spectrum (Fig. 2.5) amounts to

$$
\mathrm{E}_{\max }^{\text {Photon }}=\hbar \omega_{\max }=3.17 \mathrm{U}_{p}+\mathrm{I}_{p}
$$

This photon energy can only be achieved if the tunnel ionization of the electron takes place at a particular phase $\phi_{0}=107.96^{\circ}$ of the electric field. In this case, the reversal point $t_{u}$ is at a phase $\phi_{u}=360^{\circ}-107.96^{\circ}=252.04^{\circ}$ and the recombination $\left(t_{1}\right)$ at $\phi_{1}=342.04^{\circ}$. The bottom part of Fig. 2.6 illustrates this case.

The quantum mechanical description accompanying the semiclassical model is based on solving the non-relativistic time-dependent Schrödinger equation for an electron in the electrical field $\mathrm{E}(x, t)$ of the laser light [115]. In the position space, the equation is:

$$
i \hbar \frac{\partial \Psi(x, t)}{\partial t}=\left(-\frac{\hbar^{2}}{2 m} \frac{\partial^{2}}{\partial^{2} x}+\hat{V}(x)-e x \mathrm{E}(x, t)\right) \Psi(x, t)
$$

Here, $\Psi(x, t)$ designates the electrons wave function in the position space, $\hat{V}(x)$ represents the time-independent Coulomb potential of the atom and -ex $\mathrm{E}(x, t)$ describes the interaction of the electron with the laser field. The goal is the calculation of the time-dependent dipole moment $d(t)$ induced by the interaction of the electron with the electric field. Therefore, one has to integrate over all possible times $t_{0}$, at which an electron is set free via tunnel ionization, and take all the resulting trajectories into account. The spectrum of the high harmonics as shown in Fig. 2.5 is obtained by squaring the absolute value of the Fourier transformation $d(\omega)$ of the time dependent dipole moment $d(t)$ [116].

In oder to solve equation 2.3, a strong field approximation is made. This approximation is valid for $\mathrm{U}_{p}>\mathrm{I}_{p} \gg \hbar \omega_{0}$ and contains the following assumptions: After the liberation from the atom, the electron is considered as a free particle in an electric field. This means that the Coulomb field is neglected once the electron left the atom. The second assumption is that recombination of the electron only 
occurs back into its initial state within the atom. Other possible final states are not considered.

The semiclassical three-step model only describes the interaction of a single atom in a laser field. Collective effects in high harmonic generation, such as phase matching conditions are not considered. However, those are important for an understanding of the strength of macroscopic high harmonic emission. At the same time, the model is neither able to describe the quantum nature of the electrons interaction with the laser field, and thus of high harmonic generation in general. Despite those lacks, the three-step model is successful in describing the main properties of high harmonic generation, such as the spectral features and the polarization characteristics. This fact together with the simplicity and clearness of the model is the reason for its great success.

\subsubsection{High harmonic light source}

We generate high harmonic radiation by focusing $1.40 \mathrm{~W}$ of the $800 \mathrm{~nm}$ fundamental through a closed-end metal capillary perfused by argon gas (see Fig. 2.7). Therefore, the beam diameter of the fundamental is set to about $7-8 \mathrm{~mm}$ with an adjustable aperture right in front of the focusing lens. In our experiments, adjusting the beam diameter was found to be critical for the quality and the intensity of the high harmonic radiation. A lens with a focal length of $200 \mathrm{~mm}$ then focuses the light down to a focus size of about $50 \mu \mathrm{m}$. This corresponds to an average intensity in the focal plane of about $8 \cdot 10^{14} \mathrm{~W} / \mathrm{cm}^{2}$. We directly use the laser light to drill the required holes through the metal capillary. The capillary has an inner diameter of $3 \mathrm{~mm}$ and consists of an alloy of copper, nickel and zinc. The diameter of the resulting holes amounts to about $200 \mu \mathrm{m}$. Argon gas perfuses the capillary, which is located in the first vacuum chamber of our experimental setup. We measure the argon gas pressure at the capillary system connecting the gas cylinder with the high harmonic capillary. There, the pressure for optimal high harmonic conditions amounts between 100 mbar and 200 mbar. While the system is running, a roots pump and a rotary vane pump are maintaining the pressure in this chamber at about $10^{-2}$ mbar.

The high harmonic chamber is connected to a high vacuum chamber containing a toroidal holographic XUV grating (Jobin Yvon). A $150 \mathrm{~nm}$ thick aluminum filter (Luxel) between the two chambers blocks the fundamental radiation and simultaneously serves as a pressure shield. We use the grating to disperse the different harmonics and to select one particular harmonic. In the present experiment we select the $25^{\text {th }}$ harmonic of $800 \mathrm{~nm}$ with a photon energy of $38.7 \mathrm{eV}(32 \mathrm{~nm})$. The working range of the grating is between $34 \mathrm{eV}$ and $103 \mathrm{eV}(12-36 \mathrm{~nm})$. The 


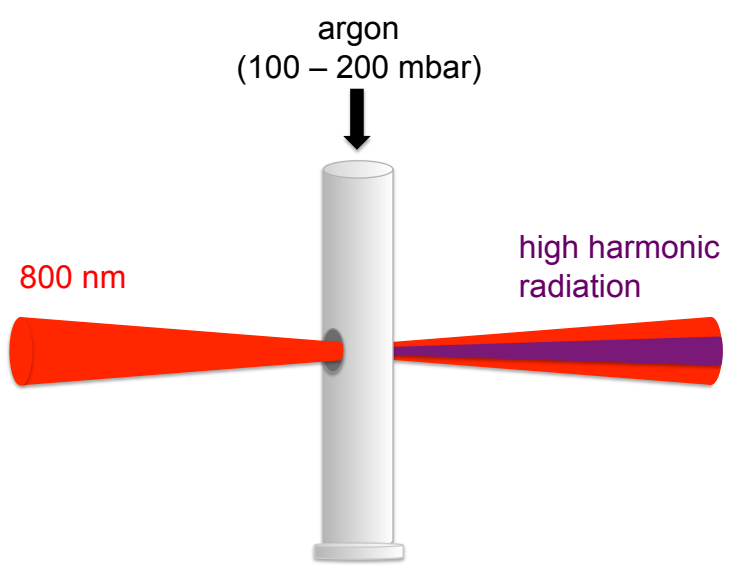

Fig. 2.7: We generate high harmonic radiation by focussing the $800 \mathrm{~nm}$ fundamental light pulses into argon gas inside a metal capillary.

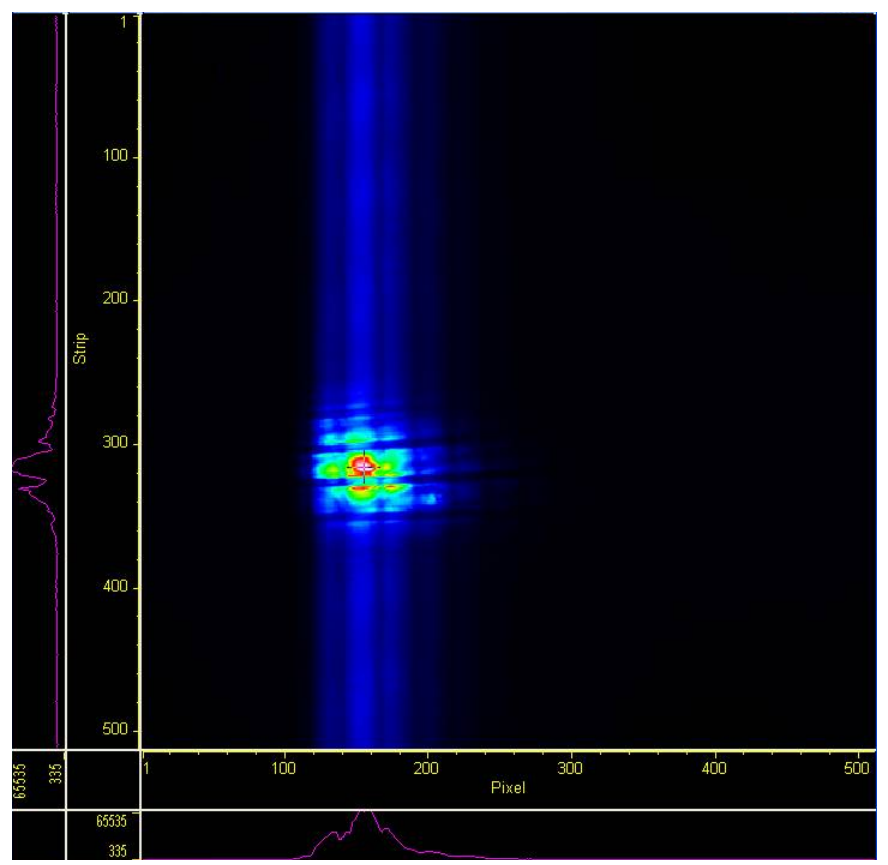

Fig. 2.8: CCD camera image of the $25^{\text {th }}$ harmonic. The vertical and horizontal lines arise from the projection of an aluminum filter in the light path between the camera and the main chamber. Evaluation of the image allows to estimate the intensity and the focus size of the high harmonic radiation. 
grating exhibits 600 lines per $\mathrm{mm}$, whereas the spacing of the grooves is computer optimized to provide a point-to-point image with a minimum of astigmatism and coma. Consequently the grooves are neither equidistant nor parallel which allows to avoid the Rowland circle rule, which applies for usual concave dispersion elements. This allows to realize a very simple monochromator scheme, in which only the grating itself rotates around an axis. The grating focuses the selected harmonic down to a spot size of about $150 \mu \mathrm{m}$ at a distance of $1414 \mathrm{~mm}$. The distance between the high harmonic capillary and the grating amounts to $1000 \mathrm{~mm}$. The drawback of this simple grating unit is the temporal broadening of the high harmonic light pulse. After the grating, the pulse length is on the order of 1 ps.

We optimize and control the high harmonic radiation by observing it with a CCD camera (Princeton instruments). The camera is attached to the main experimental chamber (see Fig. 2.3). A typical image is shown in Fig. 2.8. Evaluation of the data allows to estimate the number of high harmonic photons generated in our high harmonic light source as well as the final focus size at the position of the sample. In the $25^{\text {th }}$ harmonic we generate a total number of about $2 \cdot 10^{6}$ photons per pulse. At a photon energy of $40 \mathrm{eV}$, the efficiency of the grating in the first diffraction order amounts to $10 \%$, so that about $2 \cdot 10^{5}$ high harmonic photons per pulse reach the sample. The grating focuses the high harmonic light down to a spot size of $150 \mu \mathrm{m}$ at the position of the sample. Very recently, we were able to increase the number of photons in the $25^{\text {th }}$ harmonic by a factor of 3 by using a $300 \mathrm{~mm}$ lens to focus the fundamental into a capillary with an inner diameter of $5 \mathrm{~mm}$ (instead of a $200 \mathrm{~mm}$ lens and a $3 \mathrm{~mm}$ capillary). This significantly improved the experimental situation and paves the way for further time-resolved studies.

\subsection{Liquid phase photoelectron spectroscopy}

Photoelectron spectroscopy provides a unique insight into the electronic structure of any kind of system: atoms, molecules and complex materials [117]. Fig. 2.9 shows the basic principle of photoelectron spectroscopy. A photon with an energy of $h \nu$ knocks an electron (e.g. of an atom) out of its bound state. The energy needed to remove the electron is called its binding energy $\left(E_{b i n d}\right)$. By measuring the kinetic energy of the outgoing electron, it is possible to determine this binding energy $\mathrm{E}_{\mathrm{bind}}=h \nu-\mathrm{E}_{\mathrm{kin}}$ and thus to obtain valuable information on the electronic structure of the system under investigation. A particularly interesting question is the impact of complex environments on the electronic structure of a system. For example, how do the energetics of electronic orbitals change, when a molecule adsorbs on a solid surface? Or what is the impact of neighboring molecules in 


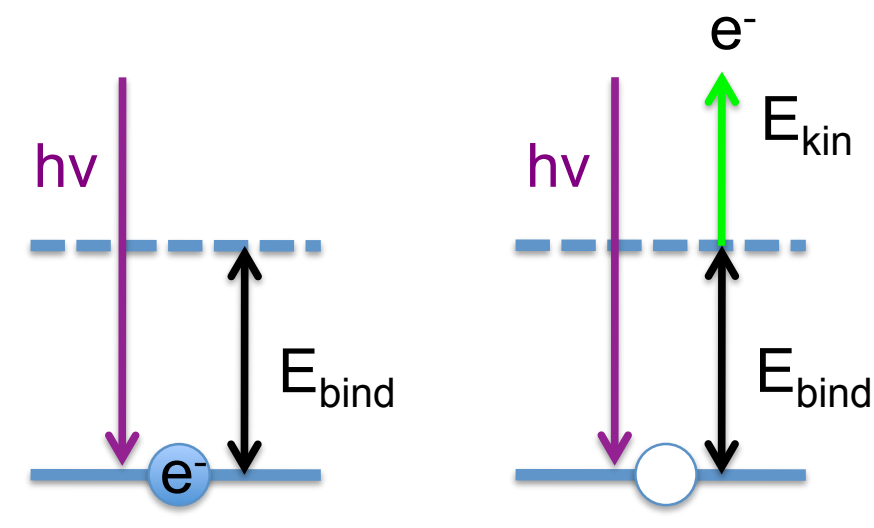

Fig. 2.9: Basic principle of photoelectron spectroscopy on the example of an atom.

condensed phases? While photoelectron spectroscopy already addressed many of these questions for gaseous, cluster and solid systems, liquid systems are a field of research which has only begun to be explored.

The reasons lie in experimental challenges. Photoelectron spectroscopy requires a sample in a high vacuum surrounding in order to avoid scattering processes of outgoing photoelectrons with gas phase molecules. While this is feasible for gas phase and solid phase studies, it constitutes a challenge for the investigation of liquids, since a volatile liquid under high vacuum conditions will either freeze or evaporate completely. In 1975 Siegbahn et al. recorded the first liquid phase photoelectron spectrum using highly concentrated salt solutions (to lower the vapor pressure) together with a special experimental wire technique [118-120]. It was not until 15 years later, when the advent of the liquid micro jet technique opened the door for further and more detailed studies on volatile liquids [121-126]. This micro jet technique was initially developed by Faubel et al. and is also used in our experimental setup.

\subsubsection{Liquid micro jet}

The distinctive feature about the liquid micro jet technique is, that it provides a free liquid surface under high vacuum conditions. A prerequisite for the formation of a stable liquid surface is a jet diameter $d_{\text {jet }}$ which is smaller than the mean free path $\lambda_{\text {mol }}$ of the respective molecules, under conditions of thermal equilibrium [121].

$$
d_{\text {jet }}<\lambda_{\text {mol }}
$$




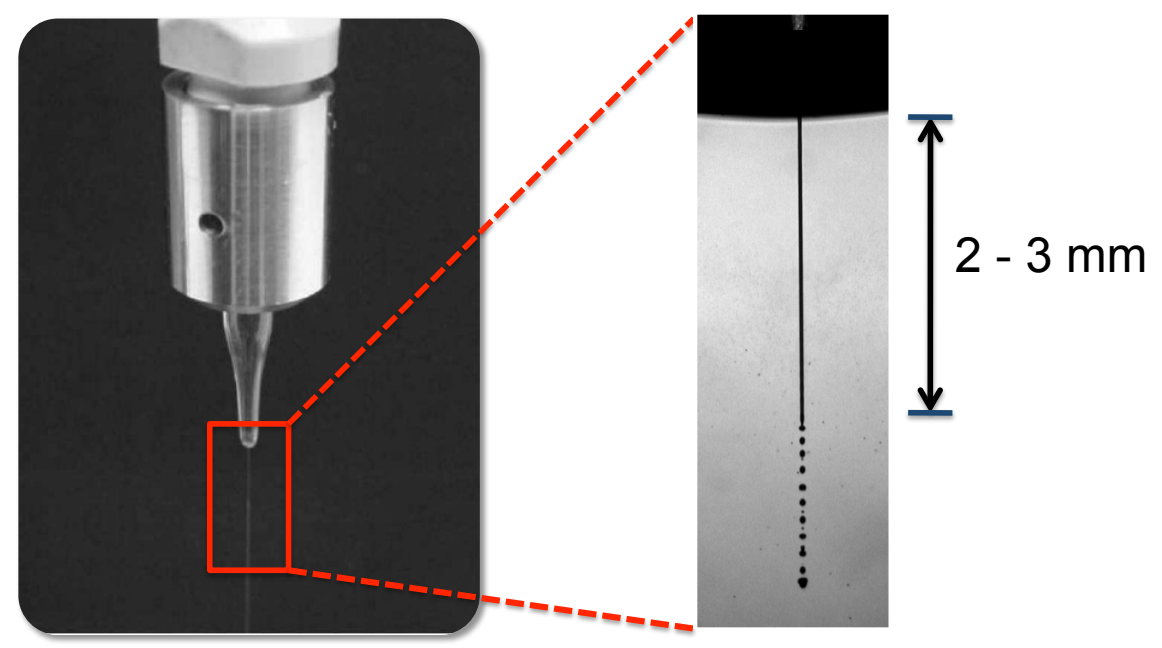

Fig. 2.10: Liquid micro jet utilized in our experiments. A plastic sealing and a screwing connect it to the capillary system of the HPLC pump. The liquid jet remains stable over a distance of about $2-3 \mathrm{~mm}$ from the nozzle tip before it breaks up into a stream of droplets.

For liquid water and a temperature of about $0^{\circ} \mathrm{C}$, a jet diameter on the order of $10 \mu \mathrm{m}$ is needed in order to fulfill this requirement. The result is a liquid surface with molecules evaporating nearly collision-free into vacuum - meaning that the density of gas phase molecules rapidly decreases with increasing distance from the liquid jet. This allows photoelectrons originating from the liquid to pass this thin and sparse gas phase (above the liquid surface) without collisions and herewith opens the door for liquid phase photoelectron spectroscopy on volatile liquids [122-126].

In our experiment we generate the micro jet by using a HPLC pump (Economy $2 / \mathrm{ED}$, Techlab Instruments) to press the liquid under investigation through a quartz glass nozzle [123]. The nozzles have typical opening diameters of $15-25 \mu \mathrm{m}$ - resulting in liquid cylindrical filaments with diameters of $12-20 \mu \mathrm{m}$ [127]. With a flux rate of $0.4 \mathrm{~mL} / \mathrm{min}$ the liquid jet remains stable over a distance of about $2-3 \mathrm{~mm}$ from the nozzle tip (see Fig. 2.10). It then breaks up into a stream of droplets which we freeze out in a trap cooled with liquid nitrogen. We use an additional cooling trap and a powerful turbo molecular pump $(1600 \mathrm{~L} / \mathrm{s}$, Pfeiffer $)$ to keep the pressure in the main chamber below $2 \cdot 10^{-5}$ mbar. In order to avoid charging effects on the liquid jet, the utilization of a very low concentrated salt solution is required [123]. To study pure liquid water, we therefore use a 0.005 $\mathrm{mol} / \mathrm{L} \mathrm{NaCl}$ solution prepared with doubly distilled water. 
An important aspect of the liquid micro jet technique is the temperature of the resulting liquid surface. When the liquid enters the vacuum through the quartz nozzle, evaporative cooling takes place which lowers the temperature of the liquid. Faubel et al. determined the temperature of the liquid jet surface by investigating the kinetic energy distribution of the evaporating molecules [121]. For a $10 \mu \mathrm{m}$ jet diameter and an initial temperature of $293 \mathrm{~K}$ they found a surface temperature of about $280 \mathrm{~K}$ for a mixture of $20 \%$ acetic acid and $80 \%$ water. In our experiments we investigate liquid jets with slightly larger diameters $(12-20 \mu \mathrm{m})$ and we also precool the liquid under investigation to a temperature of $285 \mathrm{~K}$. It is expected that due to evaporative cooling the final surface temperature amounts between $273-278 \mathrm{~K}[128]$.

The liquid surface structure and roughness are also important points for the experiments presented in this thesis. The microscopic surface structure of liquids at rest is very smooth, but roughened by thermally excited capillary waves, the liquid correspondence to solid-state transverse surface phonons. The surface roughness $\sigma$ is defined as

$$
\sigma^{2}=\left\langle[z(r)-z(0)]^{2}\right\rangle
$$

where $z(r)$ is the component of the interfacial displacement in the normal direction at a lateral position $r$. The geometrical shape of the liquid surface is primarily controlled by the surface tension $\gamma$. In the theory of thermal capillary waves, the surface roughness is related to this quantity by

$$
\sigma_{c} \sim \sqrt{\frac{k_{B} T}{\gamma}}
$$

where $T$ is the surface temperature. For liquid water at $298 \mathrm{~K}$ a capillary-waveinduced surface roughness of $0.3 \mathrm{~nm}$ was determined by grazing-incidence X-ray scattering spectrometry $[129,130]$. This value for the surface roughness approximately equals the diameter of one water molecule in the liquid (average volume per water molecule $\left.\mathrm{V} \sim 30 \AA^{3}[131]\right)$.

\subsubsection{Photoelectron spectroscopy on a liquid water surface}

The basic principle of photoelectron spectroscopy was already introduced in section 2.3. A comprehensive treatment of the theory of photoemission can be found in references $[117,132]$ and only a short introduction to the topic is presented here, 
sufficient to let readers become familiar with the most relevant phenomena in the current study. In photoelectron spectroscopy, a high energetic photon of known photon energy $h \nu$ interacts with a system M by knocking an electron out of a bound state.

$$
\mathrm{M}+h \nu \longrightarrow \mathrm{M}^{+}+e^{-}
$$

The resulting species are a cation $\mathrm{M}^{+}$and an electron with a certain kinetic energy $\mathrm{E}_{\mathrm{kin}}\left(e^{-}\right)$. The energy which is needed to remove a particular electron is called its binding energy $\mathrm{E}_{\mathrm{bind}}$ - or alternatively the ionization potential IP - and is the difference in energy between initial $\mathrm{E}(\mathrm{M})$ and final/ionized state $\mathrm{E}\left(\mathrm{M}^{+}, \mathrm{k}\right)$ of the species ( $\mathrm{k}$ is the initial level from which the electron was removed): $\mathrm{E}_{\text {bind }}=\mathrm{E}\left(\mathrm{M}^{+}, \mathrm{k}\right)$ - E(M). Energy conservation then yields:

$$
\mathrm{E}(\mathrm{M})+h \nu=\mathrm{E}\left(\mathrm{M}^{+}, \mathrm{k}\right)+\mathrm{E}_{\mathrm{kin}}\left(e^{-}\right)
$$

Measuring the kinetic energy of the outgoing electron allows to determine its initial binding energy $E_{b i n d}$ - the quantity of interest in photoelectron spectroscopy. Since this binding energy is the difference in energy between initial and final/ionized state of the system under investigation, it allows the determination of relative orbital energies. To ascertain absolute orbital energies remains a challenging topic.

Fig. 2.11 shows the valence band photoelectron spectrum of gas phase water. In the binding energy range up to $25 \mathrm{eV}$, it consists of 3 bands corresponding to ionization out of the three uppermost molecular orbitals of the water molecule: $1 b_{1}, 3 a_{1}$ and $1 b_{2}$. The signal of the $4^{\text {th }}$ valence orbital $2 a_{1}$ is located at a binding energy of $32 \mathrm{eV}$. The photoelectron bands exhibit a fine structure which results from different vibrationally excited states of the remaining cation $\mathrm{H}_{2} \mathrm{O}^{+}[134,135]$. It is particularly pronounced for the ionization out of the non-bonding $1 b_{1}$ orbital.

In 1997, Faubel et al. recorded the first valence band photoelectron spectrum of pure liquid water $[122,136,137]$. Fig. 2.12 shows a corresponding spectrum recorded with our experimental setup. The spectrum is a superposition of the water gas phase bands (Fig. 2.11) and the corresponding liquid phase bands. As it is shown in Fig. 2.12, the sharp $1 b_{1}$ signal of the gas phase is shifted towards lower binding energies and clearly broadened in the liquid. The blue shaded areas in the figure mark the binding energy ranges with considerable liquid phase intensity contribution. Similar to the $1 b_{1}$ band, also the $3 a_{1}$ and $1 b_{2}$ signals are shifted towards lower binding energy and broadened in the liquid [136,137]. This liquid - gas binding energy shift is a general feature of liquid phase photoelectron spectroscopy. 


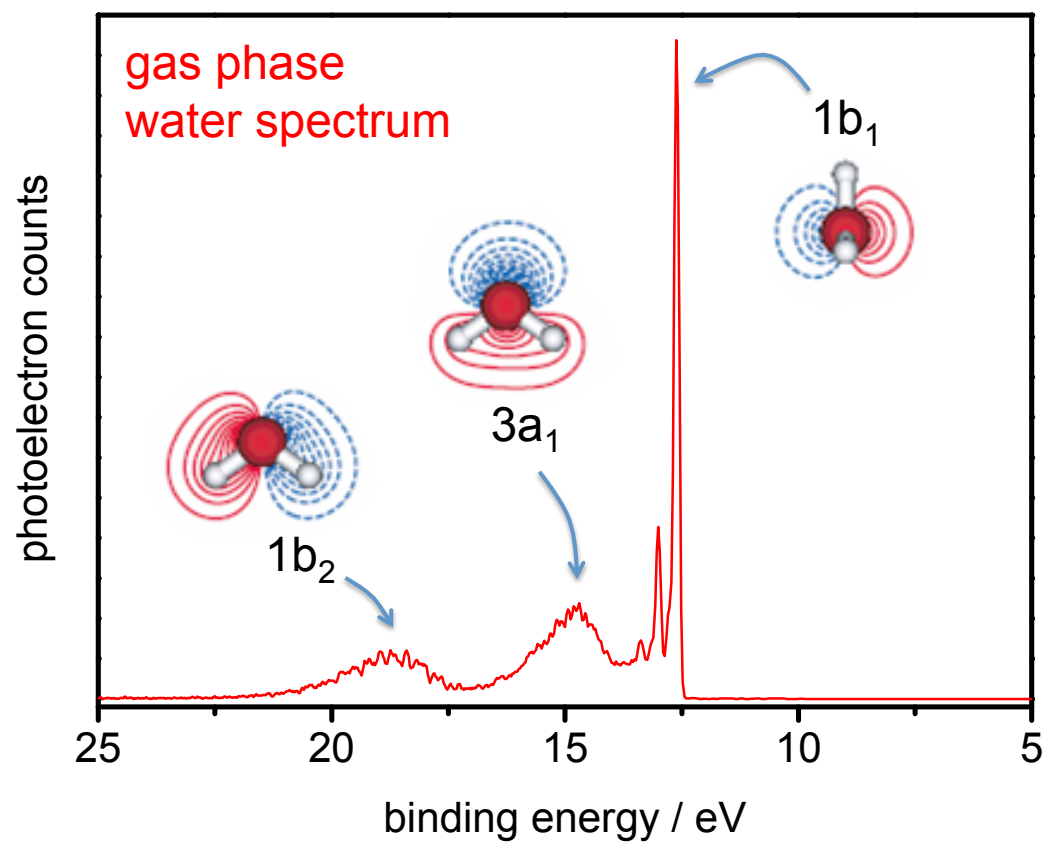

Fig. 2.11: Valence band photoelectron spectrum of gaseous water $\left(\Theta=0^{\circ}\right.$, i.e. observation direction and light polarization vector are parallel). The three bands correspond to ionization out of the three uppermost molecular orbitals: $1 b_{1}$, $3 a_{1}$ and $1 b_{2}$. Pictures of the orbitals are adopted from reference [133].

In order to understand this behavior, one has to examine the impact of surrounding species on the initial/neutral and the final/ionized state of the system under investigation (Fig. 2.13). For the case of liquid water, 2 effects have to be considered: a shift in orbital energies due to the formation of hydrogen bonds [138], and the impact of solvation on the energetics of the neutral and ionized species. In addition, the outgoing photoelectrons could be influenced by a surface potential resulting from the orientation of surface water molecules (dangling OH-bonds) [123, 131].

The main contribution to the reduced binding energy in the liquid results from the stabilization of the ionized species by the surrounding water molecules as it is schematically shown in Fig. 2.13. Already during the ionization process, neighboring water molecules shift electron density towards the ionizing species and thus stabilize the remaining cation. This shift in electron density influences the outgoing photoelectron and finally results in a lower binding energy. The amount of stabilization can be estimated from the polarization contribution of the Born-model of solvation [120,123]. Since ionization takes place on a sub femtosecond timescale, it is not affected by dissociation or molecular reorientation events following ionization. 


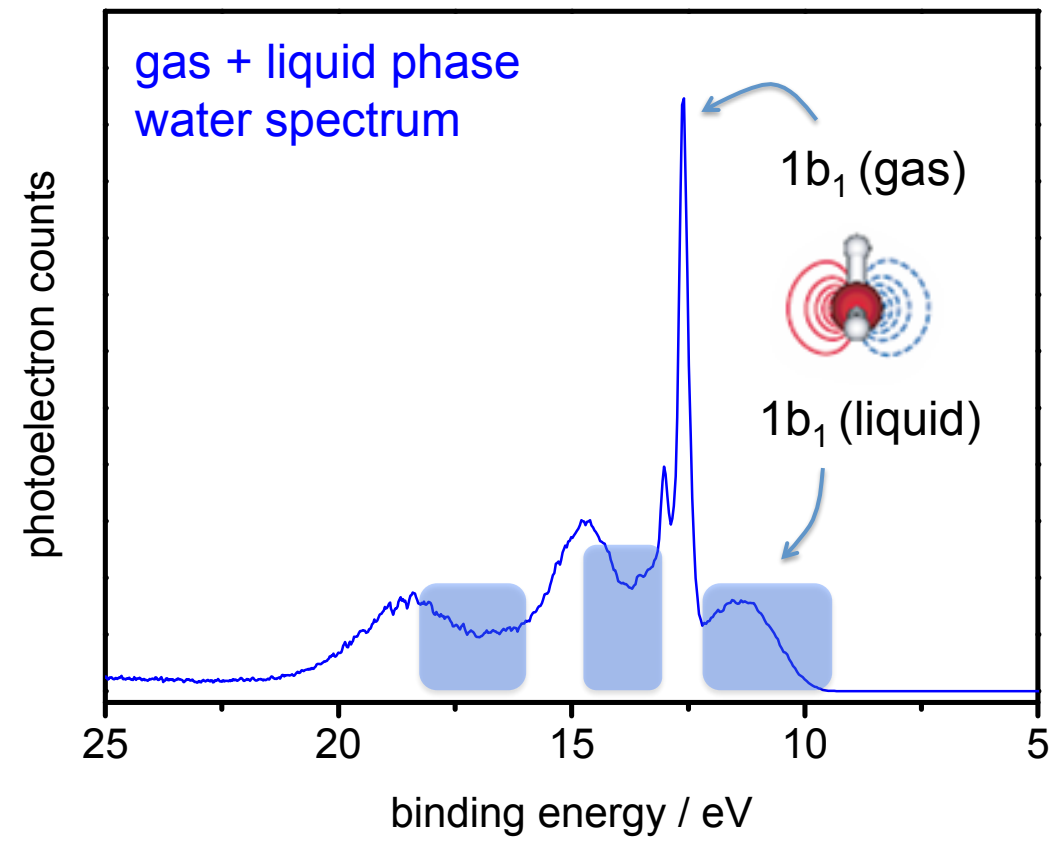

Fig. 2.12: Photoelectron spectrum recorded on a liquid water micro jet. The spectrum is a superposition of water gas phase bands and the corresponding bands of the liquid. Blue shaded areas mark the binding energy ranges with considerable liquid phase intensity contribution.

\subsubsection{Time-resolved experiments}

In 2007, our group performed the very first time-resolved liquid phase photoelectron spectroscopy experiments [21-23]. These experiments were a proof of principle and paved the way for further studies, such as the ones presented in this thesis. It is a particular challenge in each study to find the spatial and temporal overlap of the pump and probe pulse on the liquid jet. In previous experiments we observed characteristic oscillation features which allowed us to determine $\Delta t=0$ [21]. In the present study, we analyzed the impact of the $267 \mathrm{~nm}$ pump pulse on the water jet and in turn on the photoelectron spectra. The $267 \mathrm{~nm}$ pump pulse causes line shifts and deformations in the spectrum if applied with an intensity of about $1 \cdot 10^{15} \mathrm{~W} / \mathrm{m}^{2}$ or higher (see Fig. 2.14). We attribute these features to plasma formation and charging effects on the water beam. For the experiment itself, we decrease the pump pulse intensity below $5 \cdot 10^{14} \mathrm{~W} / \mathrm{m}^{2}$ in order to avoid these phenomena. By scanning the time-delay between pump and probe pulse and analyzing the onset of an effect induced by the pump pulse, we determine $\Delta t=0$ with an accuracy of about \pm 1 ps. 


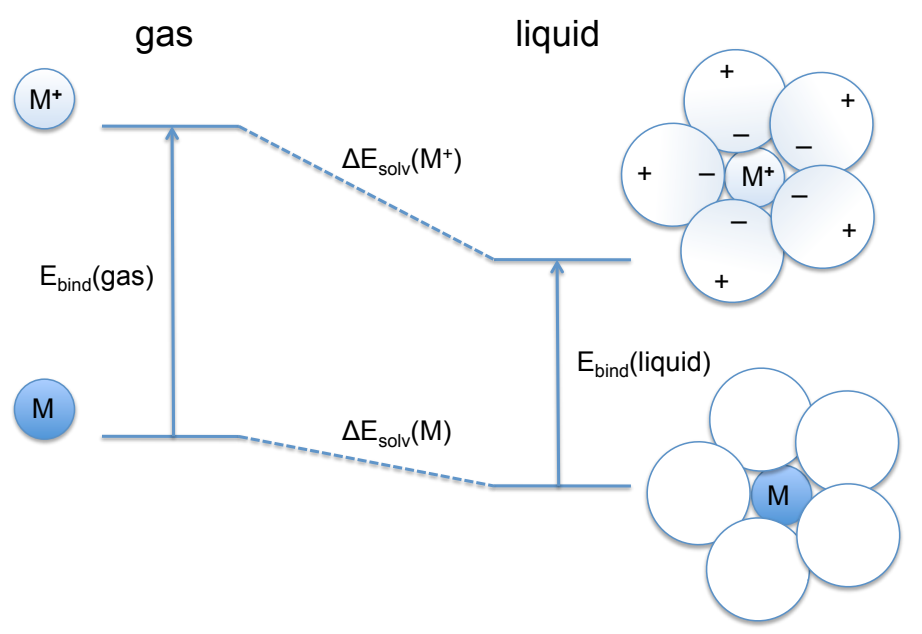

Fig. 2.13: In order to understand the reduced binding energy in condensed systems, the impact of surrounding species on the initial/neutral and final/ionized state have to be elucidated.

\subsubsection{Surface sensitivity}

An important aspect of condensed matter photoelectron spectroscopy and crucial for the present experiment is the limited escape depth of photoelectrons. Depending on the initial kinetic energy and the material properties, a photoelectron on average travels a characteristic distance between inelastic collisions in a material. This distance is called the electron inelastic mean free path (IMFP). For most materials IMFPs show a qualitatively very similar dependence on the electron kinetic energy, characterized by a minimum of about $5-10 \AA$ for kinetic energies between 50 $100 \mathrm{eV}$. For lower kinetic energies, the IMFP steeply increases and a steady but slower increase is observed for higher kinetic energies [139].

In photoelectron spectroscopy, the probing depth is limited by the occurrence of the first inelastic scattering event. Only direct or elastically scattered photoelectrons contribute to the structured part of the photoelectron spectrum. As depicted in Fig. 2.15, the measured photoelectron signal from subsurface layers declines exponentially with increasing distance from the surface. The important quantity in this context is the effective electron attenuation length (EAL). It is defined as the (shortest) distance from a starting point $\mathrm{S}$ to an arrival point $\mathrm{A}$, at which the initial electron signal is reduced to 1 /e. For weak elastic scattering - which is the case for liquid water [140] - EAL and IMFP are almost identical. However, if elastic scattering is efficient, IMFP can be significantly larger than EAL. 


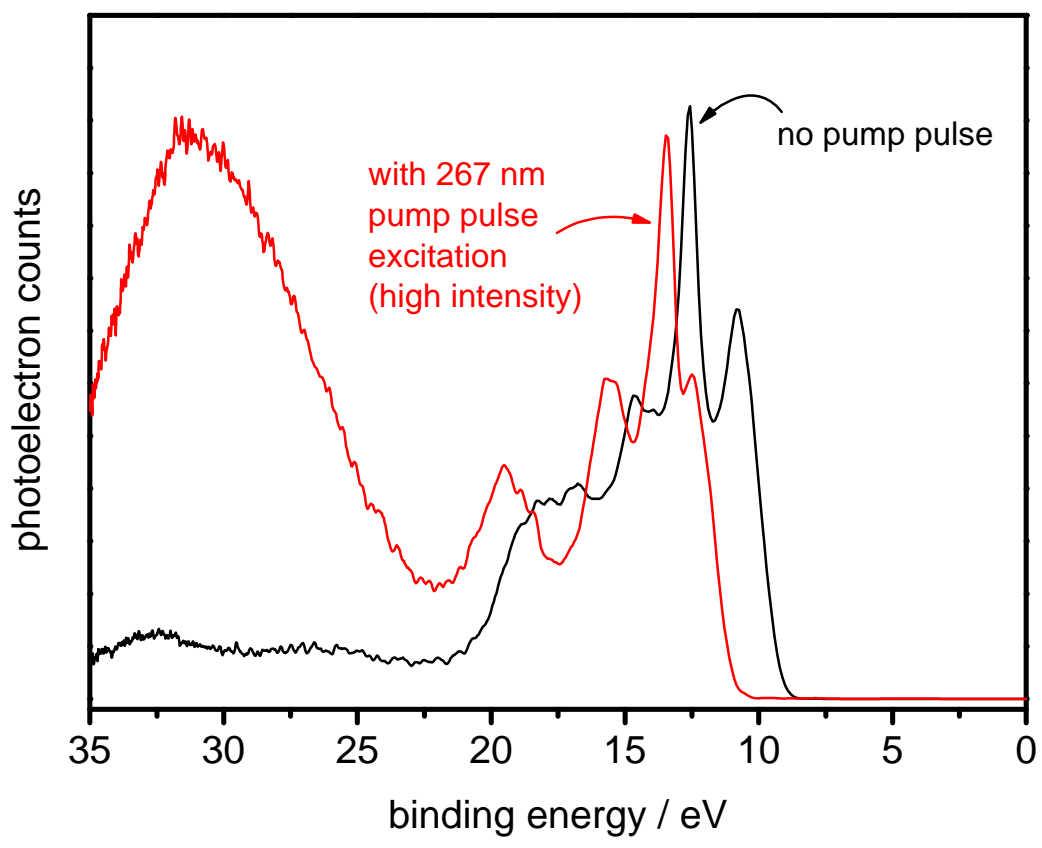

Fig. 2.14: We determine the exact overlap of the pump and probe pulses in space and time by analyzing the impact of the $267 \mathrm{~nm}$ pump pulse on the water photoelectron spectrum. The $267 \mathrm{~nm}$ pump pulse causes line shifts and deformations in the spectrum if applied with an intensity of about $1 \cdot 10^{15} \mathrm{~W} / \mathrm{m}^{2}$ or higher.

In a combined experimental and theoretical approach, Ottosson et al. determined the EAL for liquid water [140]. Using molecular dynamics (MD) simulations, they modeled the ion interfacial density profile of a NaI aqueous solution. Since iodide has a considerable propensity for the solution surface, while sodium is enriched in a following subsurface layer, the comparison with experimental photoelectron data of an aqueous $\mathrm{NaI}$ solution allows to determine a ruler for the interface, which can then be applied to water. With this experimental approach, Ottosson et al. determined the EAL for liquid water in the electron kinetic energy range between $70-900 \mathrm{eV}$ [140]. Besides, considerable computational effort has been devoted to model electron inelastic scattering in water, based on the theory of dielectric response functions [141], and experimental values of the electron mean (free) path for amorphous ice have been reported up to $100 \mathrm{eV}$ eKE [142].

In our experiments, electron attenuation lengths for electron kinetic energies of about $35 \mathrm{eV}$ are of particular interest, because we apply photon energies of 


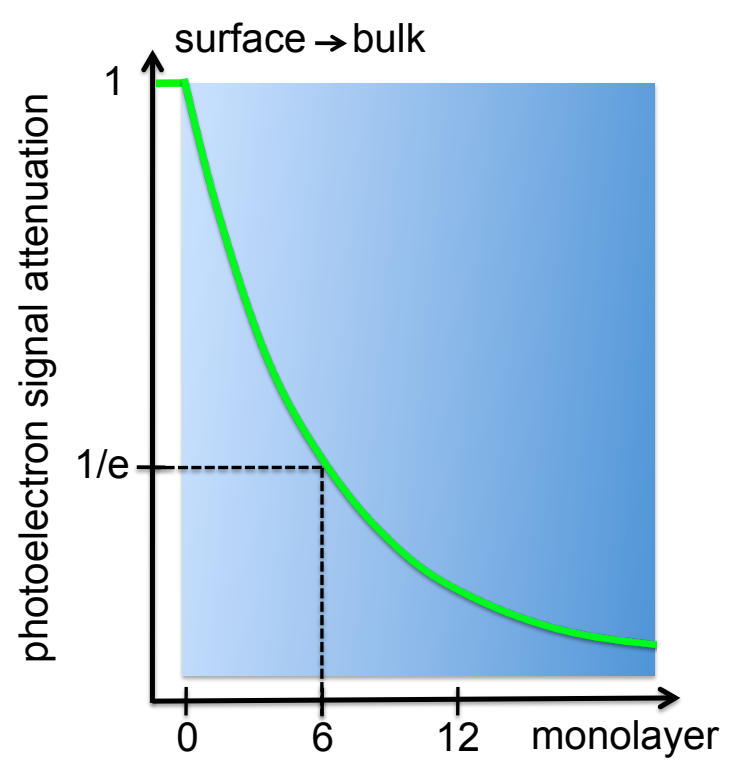

Fig. 2.15: The effective electron attenuation length EAL is defined as the distance from which the initial electron signal is reduced to 1 /e due to collision events. This figure depicts the situation representative for our experimental conditions where $\mathrm{EAL}=18 \AA$, corresponding to 6 liquid water monolayers.

$38.7 \mathrm{eV}$, and the binding energies of the hydrated electron lie between 1.6 and $3.3 \mathrm{eV}$. Ottosson et al. [140] did not determine EAL values for this kinetic energy range, but their values are close to the data for amorphous ice [140,142]. From the latter, we determine an effective electron attenuation length EAL $\approx 28 \AA$ for electrons with kinetic energies of about $35 \mathrm{eV}$. However, based on geometrical reasons the EAL value for a cylindrical jet surface is even smaller by a factor of $2 / \pi$, when photoelectrons are observed at $90^{\circ}$ with respect to the photon propagation direction [123]. As a result, the average subsurface probing depth in the cylindrical liquid jet study is reduced to $63 \%$ compared to the case of a planar target at normal emission. This means that the electron attenuation length in our experiment amounts to about $18 \AA$ for electron kinetic energies of $35 \mathrm{eV}$. Assuming a diameter of $d=3 \AA$ per liquid water monolayer [123], this EAL corresponds to 6 monolayers as depicted in Fig. 2.15.

\subsubsection{Photoelectron spectrometer and calibration}

We analyze and detect photoelectrons with a time-of-flight photoelectron spectrometer (Kaesdorf) and a multi channel plate detector (Burle) [21, 132]. The striking 
advantage of this detection method is the higher overall signal intensity, since the spectrometer detects the entire photoelectron spectrum for each laser shot. In our experiments, the liquid micro jet is located at a distance of $400-500 \mu \mathrm{m}$ in front of a skimmer (titanium, aperture $\varnothing 250 \mu \mathrm{m}$ ), which forms the entrance to the spectrometer region. A $\mu$-metal shielding excludes any magnetic fields from the interior of the spectrometer and Helmholtz coils compensate magnetic fields in the region between the liquid jet and the spectrometer entrance. Once the photoelectrons enter the spectrometer, the distance to the detection unit amounts to about $78 \mathrm{~cm}$. The electrons accomplish this distance in a time $t_{\text {flight }}$ which depends on their initial kinetic energy $\mathrm{E}_{\mathrm{kin}}$. The detector measures the arrival of electrons in time, a PCI-card (9353 100ps Time Digitizer, Ortec) reads out the detector signal (time intervals of $0.4 \mathrm{~ns}$ ), and an associated software displays the time-of-flight spectrum. We trigger the PCI-card with the signal of a photodiode installed behind the last mirror in front of the high harmonic generation chamber. Steering optics in the drift tube focus the electrons onto the detector, and an acceleration unit $(280 \mathrm{~V})$ in front of the detector enhances their detection probability. Two turbo molecular pumps (Pfeiffer) together with a membrane pump ensure a pressure of $<1 \cdot 10^{-6}$ mbar in the spectrometer unit.

To transform photoelectron spectra from the time-of-flight to the binding energy domain, we apply the following formula [21].

$$
\mathrm{E}_{\text {bind }}=\mathrm{E}_{h \nu}-\left(a \cdot\left(\frac{t_{\text {flight }}}{1 \mathrm{~ns}}\right)^{b}+c_{o}\right)
$$

Energies $\mathrm{E}_{\text {bind }}$ and $\mathrm{E}_{h \nu}$ are in $\mathrm{eV}$, the flight time $t_{\text {flight }}$ is in ns. In order to determine preliminary values for the parameters $a, b$ and $c_{o}$, we recorded gas phase photoelectron spectra with 7 different harmonics $\left(17^{\text {th }}\right.$ to $\left.29^{\text {th }}\right)$, i.e. different photon energies $h \nu$. We find that values of $a=2.528 \cdot 10^{6} \mathrm{eV}, b \approx 2.05$ and $c_{o} \approx 0 \mathrm{eV}$ are most suitable to locate the sharp $1 b_{1}$ feature in each of these spectra on the literature value of $12.61 \mathrm{eV}$. For further calibrations, we consider the value for $a=2.528 \cdot 10^{6} \mathrm{eV}$ as fixed and the obtained values for $b$ and $c_{o}$ as starting points for a calibration on literature data $[134,135]$. The parameters $b$ and $c_{o}$ are individually adjusted for every spectrum to account for two effects: (1) charging on the liquid jet and the nozzle, and (2) chromatic aberration of the grating, meaning that the wavelength spectrum of the selected harmonic is not perfectly bundled in the focus. The liquid jet with a diameter of about $15 \mu \mathrm{m}$ (and also the surrounding gas phase density which rapidly decreases with increasing distance from the liquid jet) therefore acts as a slit, 'selecting' only a certain part of the harmonics wavelength spectrum. Depending on the relative position of liquid jet and focus, the main 


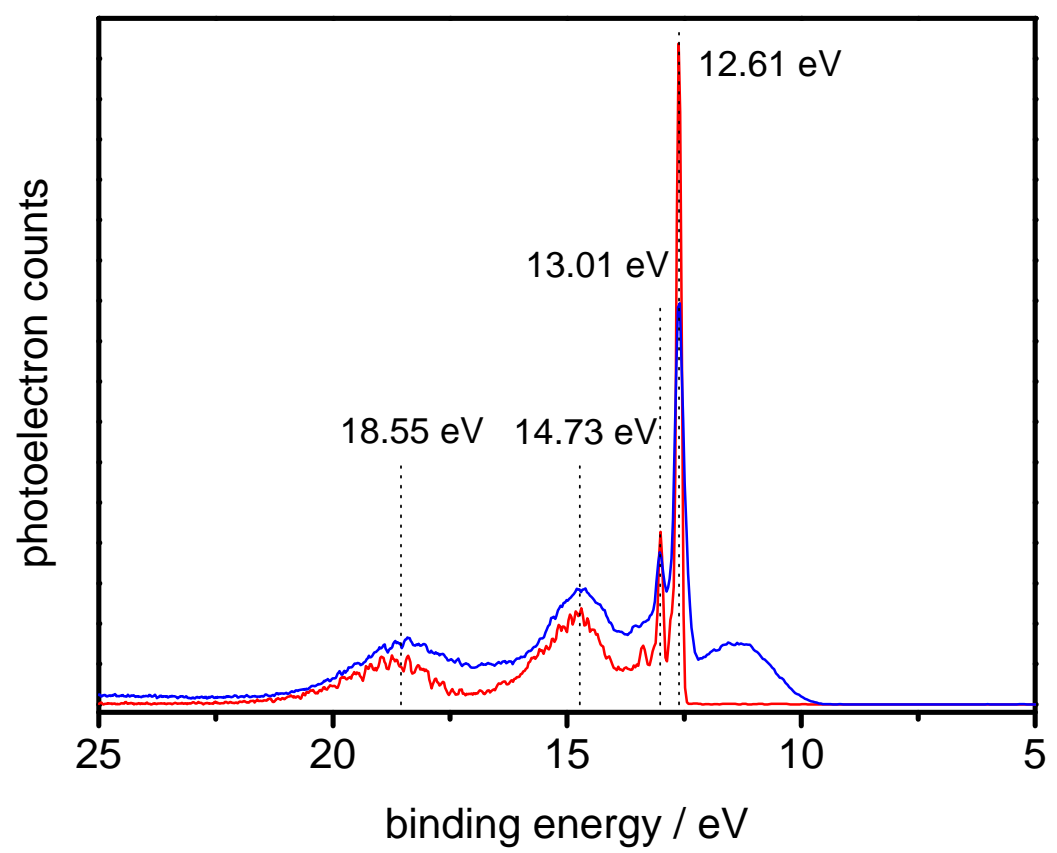

Fig. 2.16: We calibrate every spectrum on the literature values [134] by adjusting the parameters $b$ and $c_{o}$.

photon energy, and thus the entire photoelectron spectrum slightly shifts in a systematic way.

Within a series of measurements we varied the parameter $c_{o}$ to locate the $1 b_{1}$ gas phase feature of every spectrum on the literature value of $12.61 \mathrm{eV}$ (see Fig. 2.16). We find that $b$ remains unchanged for a series of measurements but slightly varies on a day-to-day basis. For all liquid phase measurements, we varied the parameter $b$ between 2.060 and 2.063 and $c_{o}$ between $-1 \mathrm{eV}$ and $+2 \mathrm{eV}$. A systematic difference in the $b$ value is observed for liquid and gas phase spectra, which is an additional indication for charging effects on the liquid jet. We record gas phase spectra by moving the liquid jet away from the skimmer aperture (spectrometer entrance) and sampling photoelectrons from the gas phase surrounding the liquid jet.

To verify our calibration procedure, we recorded mixed spectra of water vapor, $\mathrm{O}_{2}$ and $\mathrm{N}_{2}$. These spectra thus contain the water gas phase signals (see Fig. 2.11) as well as sharp lines arising from $\mathrm{O}_{2}$ and $\mathrm{N}_{2}$. We applied the calibration procedure described above to the water signals and determined the deviation of the sharp $\mathrm{O}_{2}$ and $\mathrm{N}_{2}$ lines from the literature values, respectively. For the binding energy range 
of the water signals, the calibration error is as small as $\pm 0.05 \mathrm{eV}$. The uncertainty for binding energies near $0 \mathrm{eV}$ is larger $( \pm 0.12 \mathrm{eV})$ and there is a slight tendency that binding energies determined with this calibration method are too small. The (binding) energy resolution achievable with our experimental setup amounts to $0.25 \mathrm{eV}$. 


\section{Experimental strategy and results}

Besides the experimental setup and approach presented in the previous section, it also requires a sophisticated strategy to separately investigate completely hydrated bulk electrons $e_{\text {bulk }}^{-}$and possible surface solvated electrons $e_{\text {surface }}^{-}$. Shortly, our strategy to access the properties of $e_{\text {surface }}^{-}$is to ionize pure water via the two-photon absorption of $267 \mathrm{~nm}$ light, which results in the formation of bulk and surface solvated electrons. Since our detection efficiency is highest for photoelectrons originating from the surface and declines dramatically with every layer of water molecules due to the limited mean free path of the photoelectrons, we primarily detect the signature of the surface electron in this case. In order to access the bulk solvated electrons, we accordingly have to increase their concentration and at the same time suppress the formation of $e_{\text {surface }}^{-}$. We realize this inversion by using $\left[\mathrm{Fe}(\mathrm{CN})_{6}\right]^{4-}$ complexes in aqueous solution as precursors which primarily yield $e_{\text {bulk }}^{-}$after the absorption of one $267 \mathrm{~nm}$ photon. Suppressing two-photon processes by lowering the $267 \mathrm{~nm}$ pump pulse intensity inhibits the formation of $e_{\text {surface }}^{-}$and allows for recording the signature of the bulk electron.

Fig. 3.1 presents the basic result of our studies: The photoelectron spectrum of the surface and bulk solvated electron [12]. The following sections 3.1 and 3.2 elaborate on our strategy and present detailed results on the surface and bulk solvated electron, respectively. Section 3.3 reveals the relative concentrations of $e_{\text {surface }}^{-}$and $e_{\mathrm{bulk}}^{-}$in our experiments.

\subsection{Surface solvated electron}

Fig. 3.2 outlines our strategy for accessing information on the surface solvated electron. The blue box in the figure depicts the surface (left side) and the first $\sim 20$ monolayers below the surface. We generate hydrated electrons via the two-photon absorption of $267 \mathrm{~nm}$ light (see also section 1.1.1):

$$
\mathrm{H}_{2} \mathrm{O}_{(\mathrm{aq})}+2 h \nu \longrightarrow \mathrm{OH}_{(\mathrm{aq})}+\mathrm{H}_{3} \mathrm{O}_{(\mathrm{aq})}^{+}+e_{(\mathrm{aq})}^{-}
$$



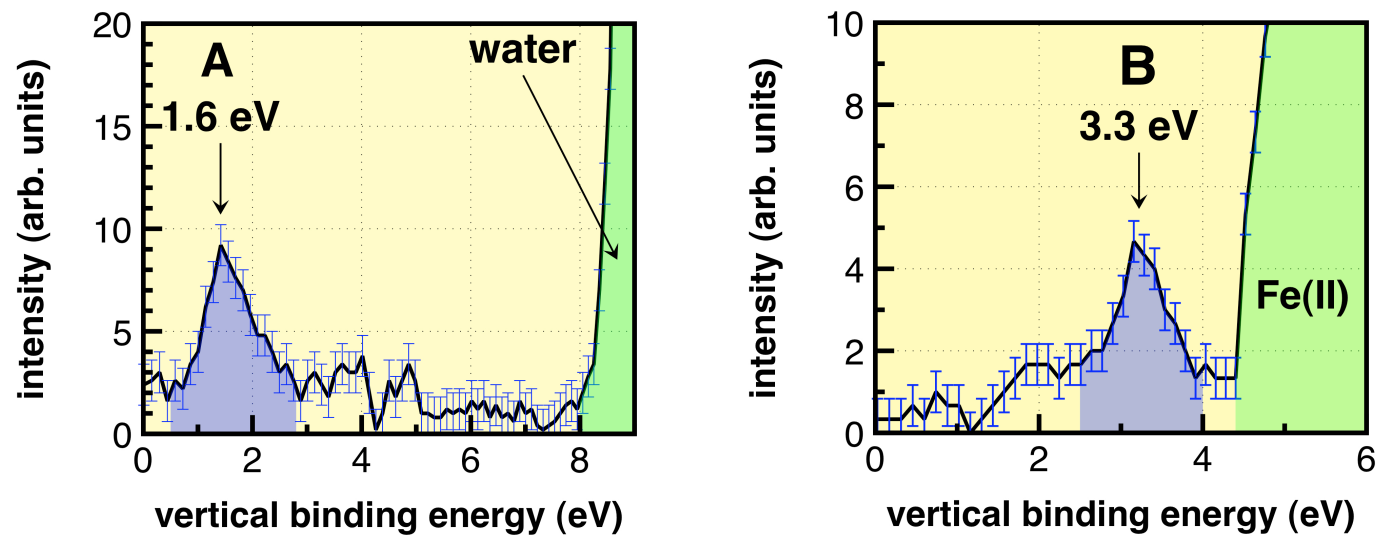

Fig. 3.1: Photoelectron spectra of surface (A) and bulk (B) solvated electrons [12].

The blue balls in Fig. 3.2 represent single water molecules as potential precursors for hydrated electrons, while the light blue balls represent the reaction products $\left(\mathrm{OH}_{(\mathrm{aq})}+\mathrm{H}_{3} \mathrm{O}_{(\mathrm{aq})}^{+}\right)$. With this approach we generate surface solvated as well as bulk solvated electrons. Subsequent to the generation of solvated electrons, we record their photoelectron spectrum with a high harmonic probe pulse $(h \nu=38.7 \mathrm{eV})$. Here, the surface sensitivity of photoelectron spectroscopy is of importance. In water, photoelectrons with kinetic energies of about $35 \mathrm{eV}\left(\approx h \nu-\mathrm{E}_{\mathrm{bind}}\left(e_{(\mathrm{aq})}^{-}\right)\right)$have an extremely short electron attenuation length (EAL) of only 6 water monolayers (see section 2.3.4). The photoelectron signal attenuation (green curve in Fig. 3.2) illustrates that the detection efficiency for surface solvated electrons is significantly higher than for bulk solvated electrons. This surface sensitivity allows us to access information on the surface solvated electron.

The left part of Fig. 3.3 shows the photoelectron spectrum recorded at time delays $\Delta t$ between $7-20 \mathrm{ps}$ after the generation of solvated electrons by the UV pump pulse. The pump pulse intensity on the liquid jet was about $5 \cdot 10^{14} \mathrm{~W} / \mathrm{m}^{2}$. The spectrum exhibits a feature located at a binding energy of $1.6 \mathrm{eV}$ which is absent without excitation by the UV pump pulse. We attribute this signal to the surface solvated electron. Every other species of the system, such as $\mathrm{OH}$ $\left(\mathrm{E}_{\text {bind }}\left(\mathrm{OH}_{(\mathrm{aq})}^{-}\right)=9.2 \mathrm{eV},[124]\right), \mathrm{H}_{3} \mathrm{O}^{+}\left(\mathrm{E}_{\text {bind }}\left(\mathrm{H}_{3} \mathrm{O}_{(\mathrm{aq})}^{+}\right)=20 \mathrm{eV},[124]\right)$ and water can be ruled out, as well as probing of any excited states, since complete relaxation occurs within less than $5 \mathrm{ps}$ [47]. The steep signal onset at about $8-9 \mathrm{eV}$ binding energy arises from the liquid water $1 b_{1}$ orbital (see section 2.3.2).

The signal of the surface solvated electron is very weak and requires a careful analysis of the noise level. We determine the noise level from the part of the 


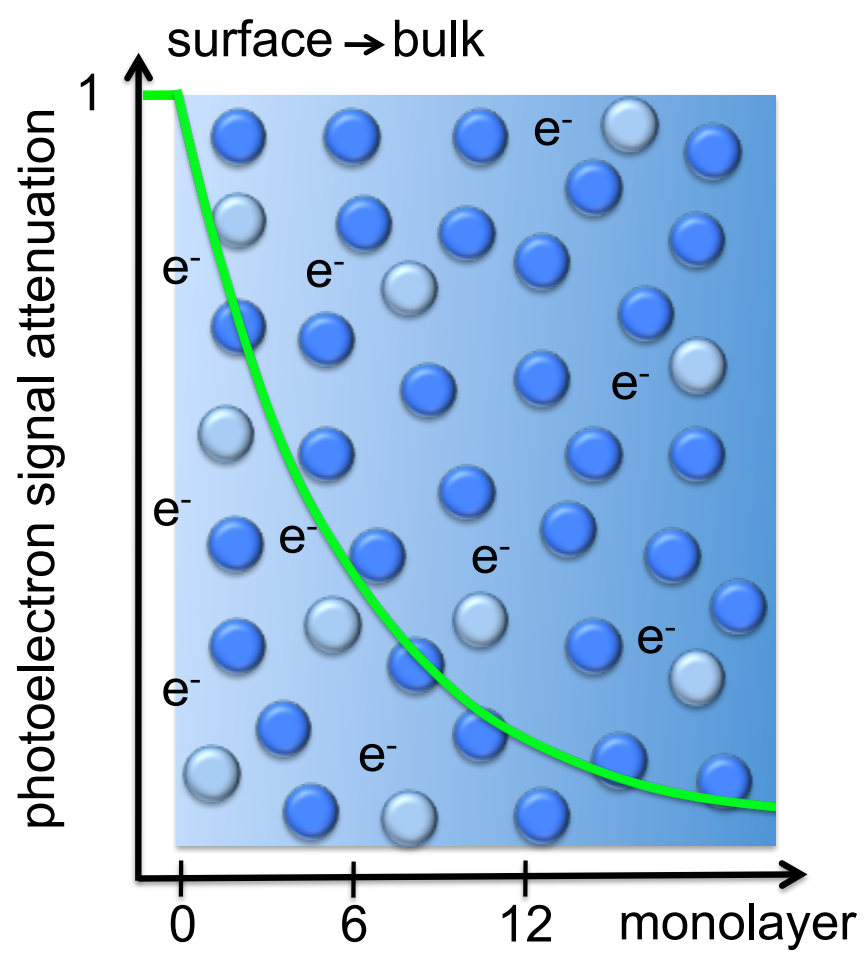

Fig. 3.2: Schematic representation of our strategy to access information on the surface solvated electron. Blue balls represent water molecules as potential precursors for hydrated electrons. Light blue balls and $e^{-}$represent the reaction products according to $\mathrm{H}_{2} \mathrm{O}_{(\mathrm{aq})}+2 h \nu \rightarrow\left(\mathrm{OH}_{(\mathrm{aq})}+\mathrm{H}_{3} \mathrm{O}_{(\mathrm{aq})}^{+}\right)+e_{(\mathrm{aq})}^{-}$. The photoelectron signal attenuation (green curve) illustrates that the detection efficiency for surface solvated electrons is significantly higher than for bulk solvated electrons.

original time-of-flight spectrum which formally belongs to the binding energy range from -10 to $0 \mathrm{eV}$. In our time-of-flight spectra, this is the interval between $192 \mathrm{~ns}$ $(-10 \mathrm{eV})$ and $214 \mathrm{~ns}(0 \mathrm{eV})$. The right part of Fig. 3.3 shows the noise spectrum (red) which belongs to the photoelectron spectrum on the left side (blue). The zero line is the mean value of the noise, while the dark grey band indicates \pm ( 1 standard deviation) from this value. Assuming a normal distribution, the dark grey band includes $68.3 \%$ of the noise. The light grey band represents \pm ( 2 standard deviations) from the mean value and accordingly includes $95.4 \%$ of the noise. Taking this into account, the feature located at a binding energy of $1.6 \mathrm{eV}$ is a clear signal.

Fig. 3.4 shows the corresponding photoelectron spectrum at a time delay $\Delta t=100 \mathrm{ps}$. As in the previous case, the spectrum exhibits a clear signal located at a binding energy of $1.5 \mathrm{eV}$. Given the weak overall signal intensity, it is difficult to draw any 

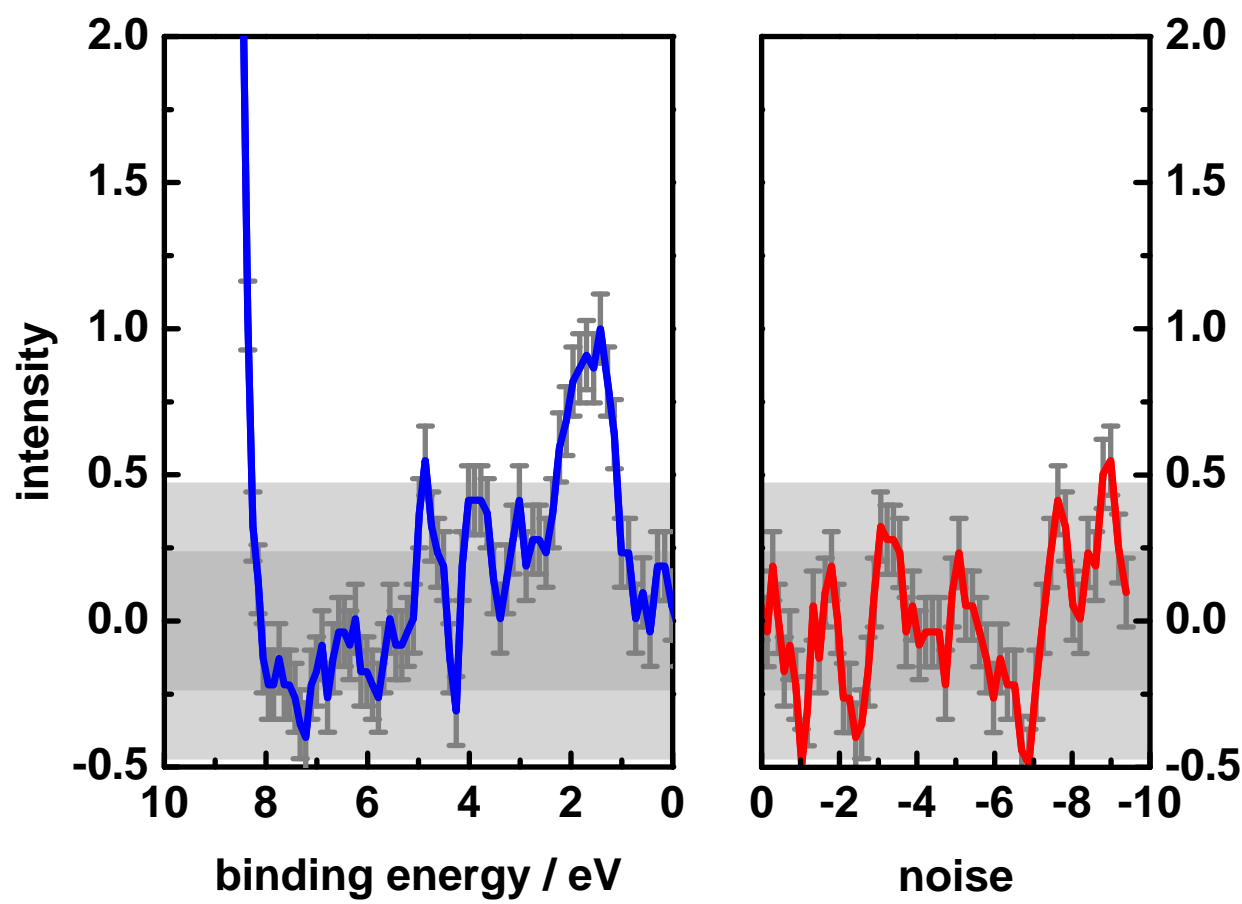

Fig. 3.3: Photoelectron spectrum of surface solvated electrons recorded at time delays between $7-20 \mathrm{ps}$ after their generation by the UV pump pulse (left). We determine the noise level from the red curve on the right. See text for details.

conclusions from a comparison with the $7-20$ ps spectrum (Fig. 3.3). However, it is interesting to compare the relative signal intensities in order to access information on the lifetime of the surface solvated electron. Therefore, the early spectrum $(7-20$ ps, Fig. 3.3) is weighted with its total acquisition time and the signal height is set to an intensity of 1 . The late spectrum (100 ps, Fig. 3.4) is accordingly weighted with the relative acquisition time so that the signal intensities can be directly compared. Interestingly, the signal does not decay on a $100 \mathrm{ps}$ timescale, indicating that the lifetime of surface solvated electrons exceeds 100 ps.

Since the two surface spectra shown in Fig. $3.3(\Delta t=7-20$ ps $)$ and Fig. $3.4(\Delta t=$ $100 \mathrm{ps}$ ) are almost identical, we add them to yield a better signal to noise ratio. Fig. 3.5 shows the result, which is the photoelectron spectrum of the surface solvated electron. The signal is located at a position of $1.6 \mathrm{eV}$ and possesses a full width at half maximum FWHM of $1.3 \mathrm{eV}$. This width primarily reflects the distribution of hydration configurations and there may be additional contributions associated with final state effects of the photoemission process. 

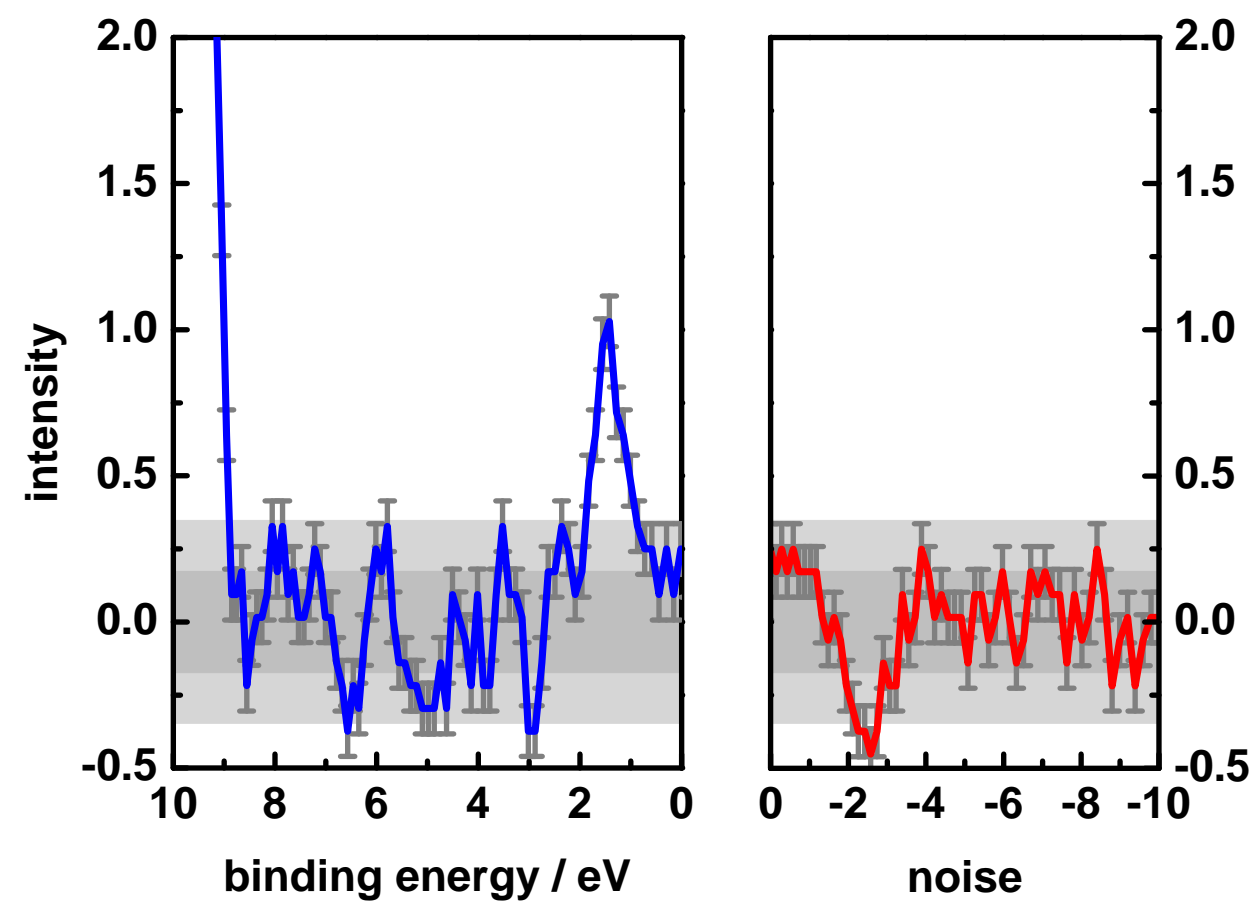

Fig. 3.4: Photoelectron spectrum of surface solvated electrons recorded at time delay of 100 ps after their generation by the UV pump pulse (left). We determine the noise level from the red curve on the right. The signal intensity can be directly compared to the signal intensity in Fig. 3.3.

It is interesting to speculate about the mechanism for the formation of surface solvated electrons. Up to now, information on the energetics of surface water molecules $[131,143]$ are insufficient to conclude on a mechanism or to judge if the bulk mechanisms (section 1.1.1) can be applied to ionization processes near the surface. However, the following is a conceivable scenario: In the two-photon process we use $2 \times 267 \mathrm{~nm}=9.3 \mathrm{eV}$ to ionize water molecules. In this ionization energy range, the proton-coupled electron transfer mechanism competes with autoionization of the molecular excited state of water (see section 1.1.1, [47,48]). The first mechanism bypasses the conduction band and directly transfers the excited electron into a preexisting trap state. In this context, the surface might offer a number of suitable trap states, since water molecules with dangling $\mathrm{OH}$ bonds are good candidates for the trapping of electrons $[87,144]$ and are abundant on a liquid water surface [131]. 

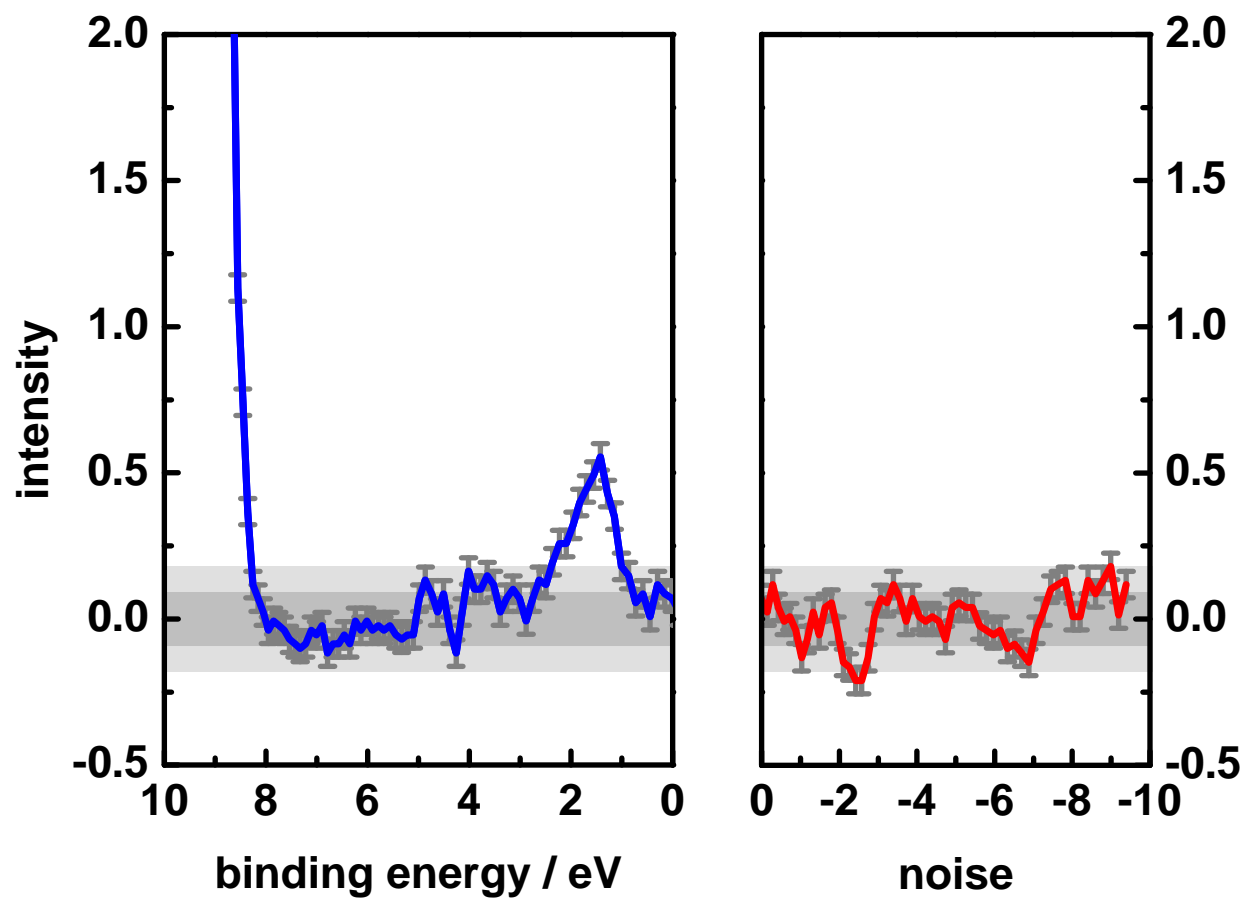

Fig. 3.5: Photoelectron spectrum of the surface solvated electron. The photoemission signal is located at a binding energy of $1.6 \mathrm{eV}$ and possesses a width of $\mathrm{FWHM}=$ $1.3 \mathrm{eV}$. The noise level is determined from the red spectrum on the right.

\subsection{Bulk solvated electron}

In order to measure the photoelectron spectrum of the bulk hydrated electron, we have to defeat the surface sensitivity of photoelectron spectroscopy. This means that we need to find a suitable precursor for solvated electrons, which exclusively generates bulk hydrated electrons and at the same time allows to suppress the formation of $e_{\text {surface }}^{-}$Fig. 3.6 depicts this situation. We find that an aqueous solution of $\mathrm{K}_{4}\left[\mathrm{Fe}(\mathrm{CN})_{6}\right]$, in which hydrated electrons are generated via photodetachment from the ferrocyanide anion $\left[\mathrm{Fe}(\mathrm{CN})_{6}\right]^{4-}$ is ideal for this purpose:

(1) We use an aqueous solution of $\mathrm{K}_{4}\left[\mathrm{Fe}(\mathrm{CN})_{6}\right]$ at a concentration of $0.5 \mathrm{~mol} / \mathrm{L}$. At this concentration, the $\left[\mathrm{Fe}(\mathrm{CN})_{6}\right]^{4-}$ anions are not surface active and rather repelled from the surface [145]. Electrons generated via photodetachment from this complex are thus primarily bulk solvated electrons. Photodetachment proceeds via excitation into a charge-transfer-to-solvent (CTTS) band and thus involves the 


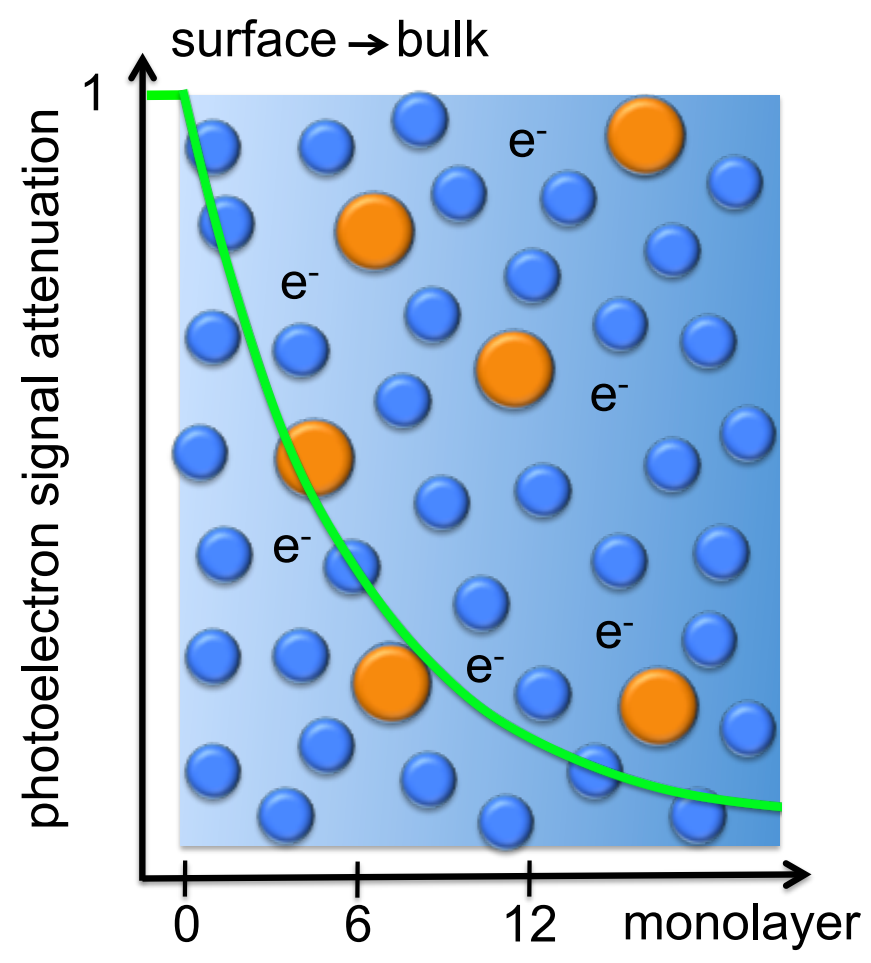

Fig. 3.6: The figure shows our strategy to access the photoelectron spectrum of the bulk solvated electrons. In a one-photon $(267 \mathrm{~nm})$ process, we photodetach electrons from $\left[\mathrm{Fe}(\mathrm{CN})_{6}\right]^{4-}$ complexes in aqueous solution, which results in the formation of bulk solvated electrons and $\left[\mathrm{Fe}(\mathrm{CN})_{6}\right]^{3-}$ complexes (orange balls). Suppressing two-photon processes by lowering the $267 \mathrm{~nm}$ pump pulse intensity inhibits the formation of $e_{\text {surface }}^{-}$and allows us to record the signature of the bulk electron.

conduction band of the liquid. Electrons in the conduction band are formally free and have the possibility to relax into a hydration configuration with maximum stability, which is a hydration cavity in the bulk. The conduction band may further be disrupted near the surface, which could additionally hamper the formation of surface solvated electrons in this case.

(2) In aqueous solution, the ferrocyanide anion strongly absorbs at $267 \mathrm{~nm}$. Excitation occurs into a charge-transfer-to-solvent (CTTS) band and results in the formation of a bulk hydrated electron (see point (1)) at a high quantum yield (see section 1.1.2). Since this excitation is a one-photon process - in contrast to the two-photon process resulting in the formation of bulk and surface solvated electrons (section 3.1) - lowering the pump pulse intensity allows us to suppress two-photon processes and thus the formation of surface solvated electrons. We lowered the 

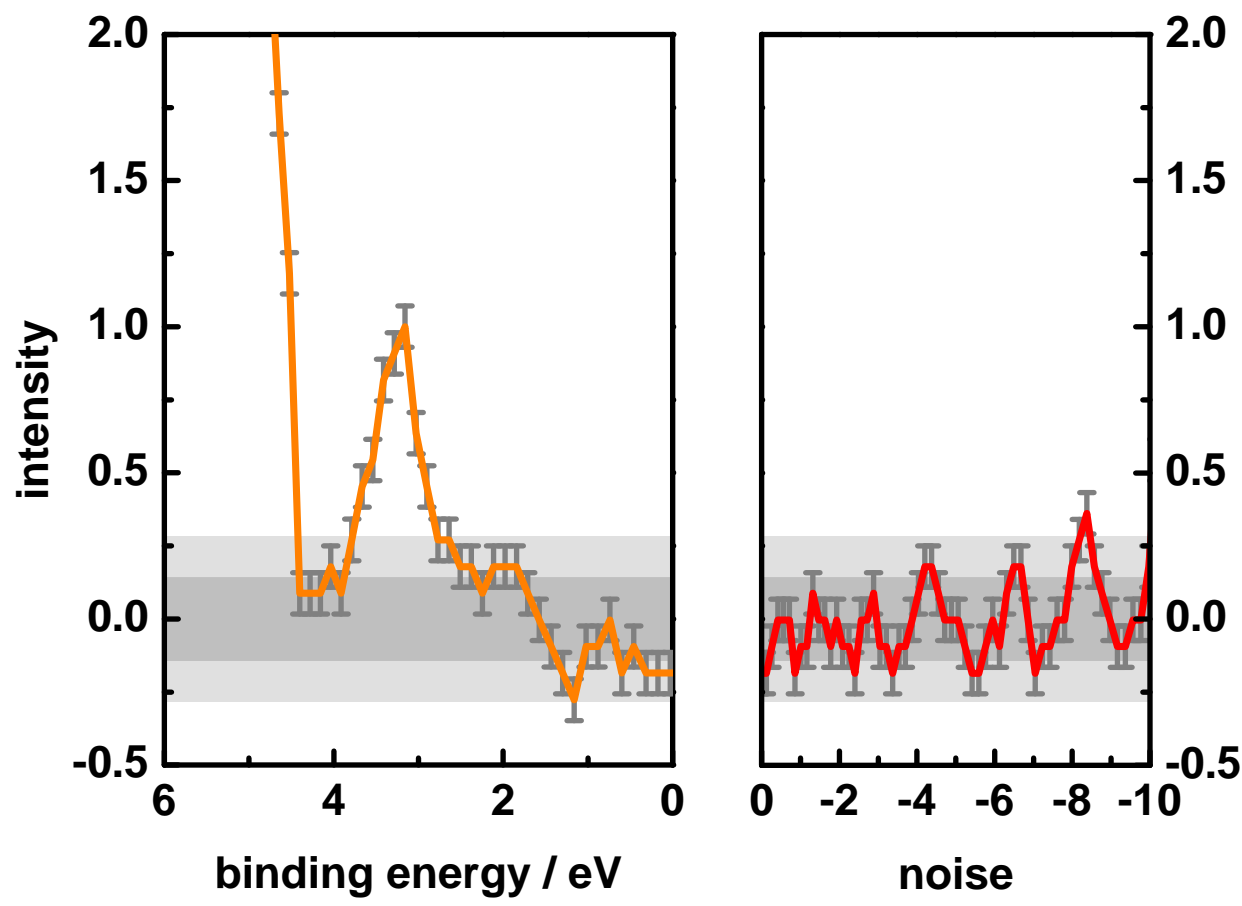

Fig. 3.7: The left part of the figure shows the photoelectron signal of the bulk solvated electron $\left(\mathrm{E}_{\mathrm{bind}}=3.3 \mathrm{eV}\right)$ recorded at a time delay of $100 \mathrm{ps}$ after the generation of hydrated electrons via the CTTS excitation of $\left[\mathrm{Fe}(\mathrm{CN})_{6}\right]_{(\mathrm{aq})}^{4-}$. We determine the noise level from the red curve on the right.

pump-pulse intensity by a factor of 10, below the threshold for two-photon ionization of about $5 \cdot 10^{13} \mathrm{~W} / \mathrm{m}^{2}$ [146]. We achieved this with a lower pulse energy and a larger focus size, as well as by extending the pulse duration.

(3) In aqueous solution, the majority of the $\left[\mathrm{Fe}(\mathrm{CN})_{6}\right]^{4-}$ complexes are paired with one, two or three $\mathrm{K}^{+}$ions. At a concentration of $0.5 \mathrm{~mol} / \mathrm{L}$, the major species in solution is $\mathrm{K}_{3}\left[\mathrm{Fe}(\mathrm{CN})_{6}\right]^{-}$resulting in a neutral $\mathrm{K}_{3}\left[\mathrm{Fe}(\mathrm{CN})_{6}\right]$ complex after photodetachment [59]. In addition, experiments revealed that the ejected electron starts out on average five solvent shells (about $15 \AA$ ) away from the oxidized iron complex (section 1.1.2). Taken together the long ejection length and the low average charge of the oxidized iron complex suggests that the hydrated electron is hardly influenced by its parent complex or the relatively high salt concentration. This is further supported by the experimental finding that the energy positions of iodide and water photoelectron signals in an aqueous sodium iodide solution 


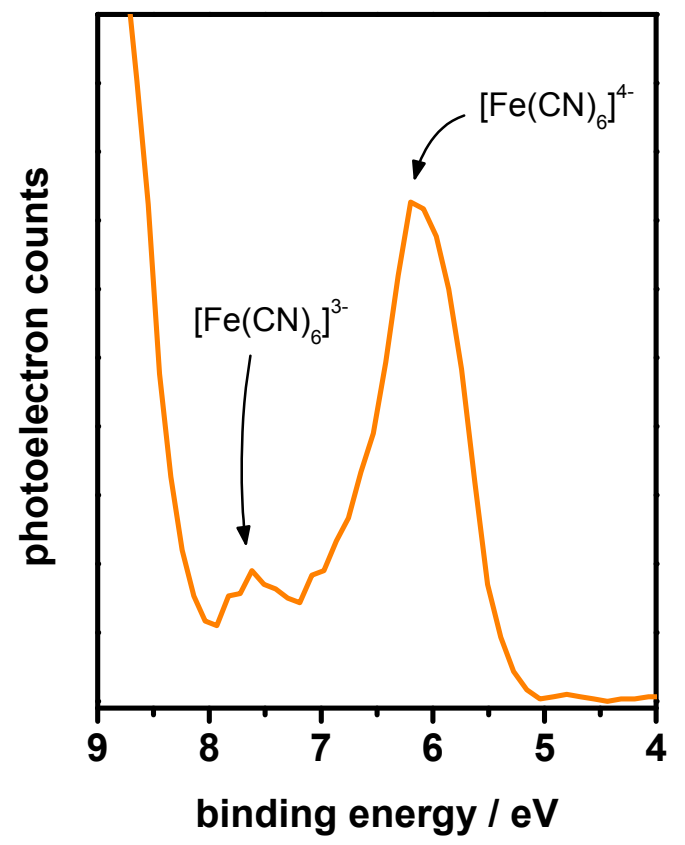

Fig. 3.8: After excitation by the UV pump pulse, we observe the signals arising from the $\left[\mathrm{Fe}(\mathrm{CN})_{6}\right]_{(\mathrm{aq})}^{4-}$ complex $\left(\mathrm{E}_{\text {bind }}=6.2 \mathrm{eV}\right)$ as well as of the corresponding oxidized complex $\left[\mathrm{Fe}(\mathrm{CN})_{6}\right]_{(\mathrm{aq})}^{3-}\left(\mathrm{E}_{\text {bind }}=7.6 \mathrm{eV}\right)$. The latter signal is absent without excitation by the UV pump pulse.

do not change as a function of salt concentration $(0.1-12 \mathrm{~mol} / \mathrm{L}$ sodium iodide in aqueous solution) [123]. In addition, Bonin et al. investigated the influence of different salts on the absorption spectrum ( $s \rightarrow p$ transition) of the hydrated electron [147]. For salt concentrations of $0.5 \mathrm{~mol} / \mathrm{L}$, the blue shift of the absorption maximum amounts to less than $0.05 \mathrm{eV}$. Molecular dynamics simulations show that the observed blue shift can primarily be attributed to a destabilization of the p-like state [148]. This can be taken as another indication that the energetics of the hydrated electron are hardly influenced by the presence of salt at a concentration of $0.5 \mathrm{~mol} / \mathrm{L}$.

The left part of Fig. 3.7 shows the photoelectron spectrum recorded at a time delay of 100 ps after the generation of hydrated electrons via the CTTS excitation of $\left[\mathrm{Fe}(\mathrm{CN})_{6}\right]^{4-}$ complexes in aqueous solution: 


$$
\left[\mathrm{Fe}(\mathrm{CN})_{6}\right]_{(\mathrm{aq})}^{4-}+h \nu \longrightarrow\left[\mathrm{Fe}(\mathrm{CN})_{6}\right]_{(\mathrm{aq})}^{3-}+e_{(\mathrm{aq})}^{-}
$$

As in the case of the surface solvated electron, the noise level and the grey noise bands are determined from the spectrum shown on the right (see section 3.1). The spectrum shows a clear signal located at a binding energy of $3.3 \mathrm{eV}$, which is absent without excitation by the UV pump pulse. The signal width amounts to $\mathrm{FWHM}=0.8 \mathrm{eV}$ and primarily reflects the distribution of hydration configurations. We attribute this signal to the photoelectron spectrum of the bulk hydrated electron. Every other species of the system can be ruled out as a source of this signal, as well as probing of any excited states since complete relaxation occurs within a few picoseconds [59]. The steep signal onset at about $4.5 \mathrm{eV}$ arises from the first photoelectron band of the $\left[\mathrm{Fe}(\mathrm{CN})_{6}\right]^{4-}$ complex, which peaks at a binding energy of $6.2 \mathrm{eV}$ (see also Fig. 3.8).

According to equation 3.2, photodetachment from the $\left[\mathrm{Fe}(\mathrm{CN})_{6}\right]_{(\mathrm{aq})}^{4-}$ complex also results in the formation of the corresponding oxidized complex $\left[\mathrm{Fe}(\mathrm{CN})_{6}\right]_{(\mathrm{aq})}^{3-}$. Fig. 3.8 shows that after excitation by the UV pump pulse, we indeed observe the signal arising from the oxidized species. While the signal of $\left[\mathrm{Fe}(\mathrm{CN})_{6}\right]_{(\mathrm{aq})}^{4-}$ is located at a binding energy of $6.2 \mathrm{eV}$, its oxidized counterpart peaks at $7.6 \mathrm{eV}$. Both binding energies are in agreement with the literature values [136].

\subsection{Relative concentrations of bulk and surface solvated electrons}

Fig. 3.9 shows the photoelectron spectrum for the bulk (right) and surface (left) solvated electron. The latter is the sum of the two surface spectra shown in Fig. 3.3 $(\Delta t=7-20 \mathrm{ps})$ and Fig. $3.4(\Delta t=100 \mathrm{ps})$. The bulk spectrum is weighted with its total acquisition time and the signal height is set to an intensity of 1 . The surface spectrum is accordingly weighted with the relative acquisition time, so that the signal intensities can be directly compared. Evaluation of the signal heights reveals that the bulk signal is more intense by a factor of 1.8. This result is of interest, when analyzing our possibility to switch between measuring surface and bulk solvated electrons. With our experimental conditions we are apparently in the range of a very delicate balance between detection sensitivity and concentration. It is also interesting to discuss our electron attenuation length (EAL) of 6 water monolayers (see section 2.3.4) in the context of the obvious possibility to measure surface solvated electrons (without a significant signal contribution from bulk solvated electrons). This might indicate that the first monolayers of the liquid are void of 

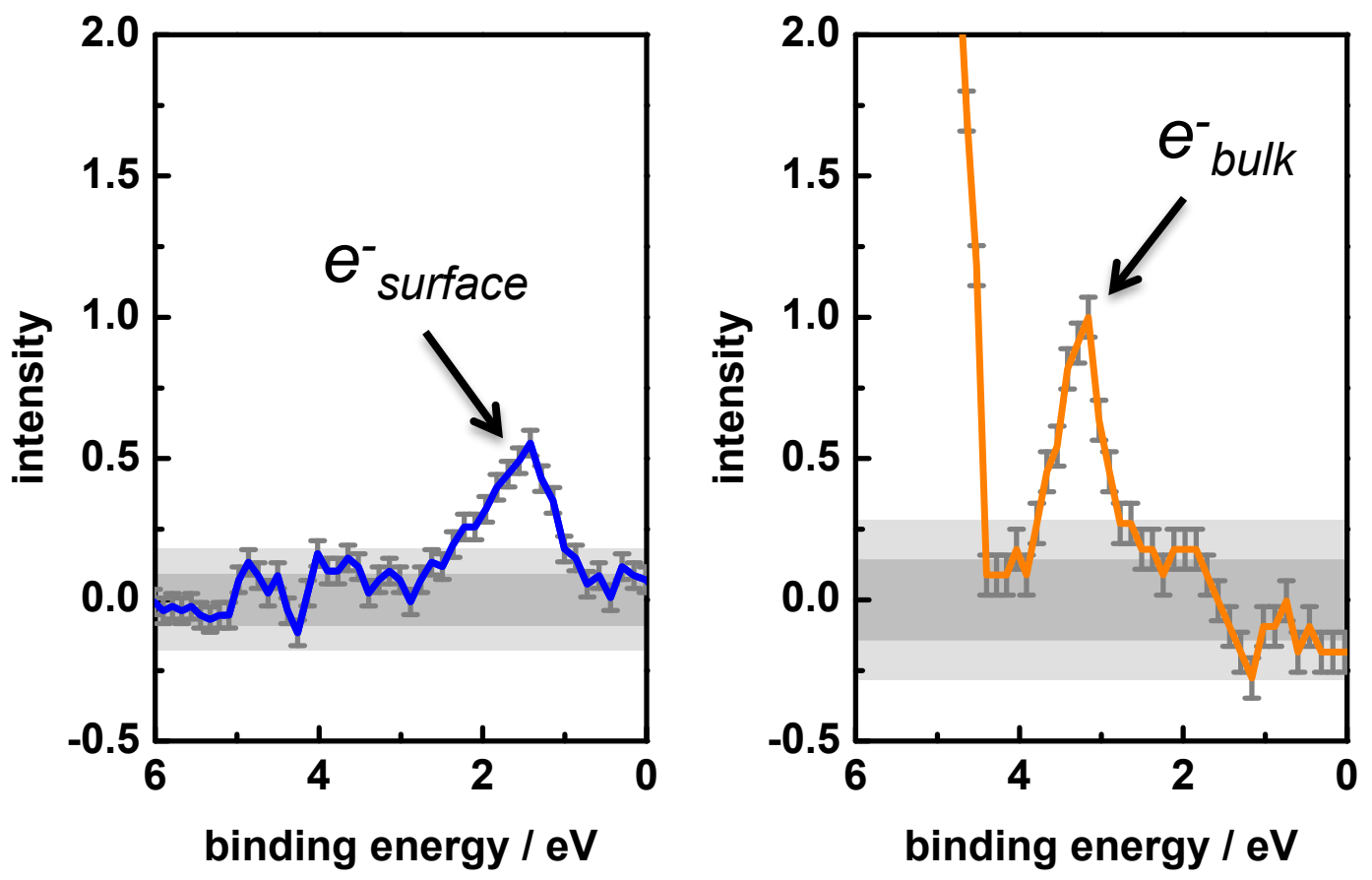

Fig. 3.9: Photoelectron spectra of bulk and surface solvated electrons. The signals are weighted with their total acquisition times, whereas the signal height of the bulk solvated electron is set to 1 .

bulk solvated electrons. However, since the determination of exact values for the EAL in liquid water is particularly difficult, it might also be that the 'real' electron attenuation length in liquid water is smaller than 6 monolayers [123].

Further signal evaluation even allows a rough estimate on the relative concentrations of bulk and surface solvated electrons in both cases. We assume that the photoionization cross sections of the two species are the same and that we detect surface solvated electrons with a probability of 1 , while we detect bulk solvated electrons with an average probability of $1 / \mathrm{e} \approx 1 / 3$. The latter arises from the photoelectron signal attenuation presented in section 2.3.4. Given our noise levels, we further assume that we are not able to detect any signals which are 3 times lower in intensity than the signals we are observing for the surface and bulk electrons. Applied to the two cases, this gives: 


\section{Two-photon ionization of water}

We observe a signal arising from surface solvated electrons which is at least 3 times more intense than the signal of bulk solvated electrons in this spectrum (observed signal). We detect surface solvated electrons with a probability of 1 , while we detect bulk solvated electrons with an average probability of $1 / 3$ (detection probability). Taken together, a situation in which the amount of surface solvated electrons equals the amount of 'accessible' bulk solvated electrons - which are bulk electrons within the first few monolayers of the liquid - is in line with our observation (concentration).

Two-photon ionization of water

\begin{tabular}{ccc}
\hline & surface & bulk \\
\hline observed signal & 3 & $<1$ \\
detection probability & 3 & 1 \\
concentration & 1 & $<1$ \\
\hline
\end{tabular}

\section{Photodetachment from $\left[\mathrm{Fe}(\mathrm{CN})_{6}\right]^{4-}$ complexes}

In this case, a situation in which the amount of accessible bulk solvated electrons is at least 9 times higher than the amount of surface solvated electrons is in line with our observation.

\begin{tabular}{ccc} 
Photodetachment from $\left[\mathrm{Fe}(\mathrm{CN})_{6}\right]^{4-}$ \\
\hline & surface & bulk \\
\hline observed signal & $<1$ & 3 \\
detection probability & 3 & 1 \\
concentration & $<1$ & 9 \\
\hline
\end{tabular}

Fig. 3.10 shows an entire photoelectron spectrum together with the signal of the bulk solvated electron. The spectrum in the binding energy range between $5 \mathrm{eV}$ and $30 \mathrm{eV}$ is one single pump-probe photoelectron spectrum which assures that the different signals are well resolved. The spectrum of the bulk solvated electron 
shown between $0 \mathrm{eV}$ and $4.5 \mathrm{eV}$ binding energy is an average of several pump probe spectra. Intensities are scaled accordingly. The intensity scale is logarithmic in order to allow a direct comparison of signal intensities. It is particularly interesting to compare the signal height of the solvated electron with the signal height of the water gas phase $1 b_{1}$ signal (highest signal in the spectrum). The ratio amounts $1: 50000$ for the bulk electron, while we evaluate about $1: 100000$ for the surface solvated electron. This result is impressive concerning the detection sensitivity and signal-to-noise ratio and clearly shows the excellent dynamical range of our experimental setup.

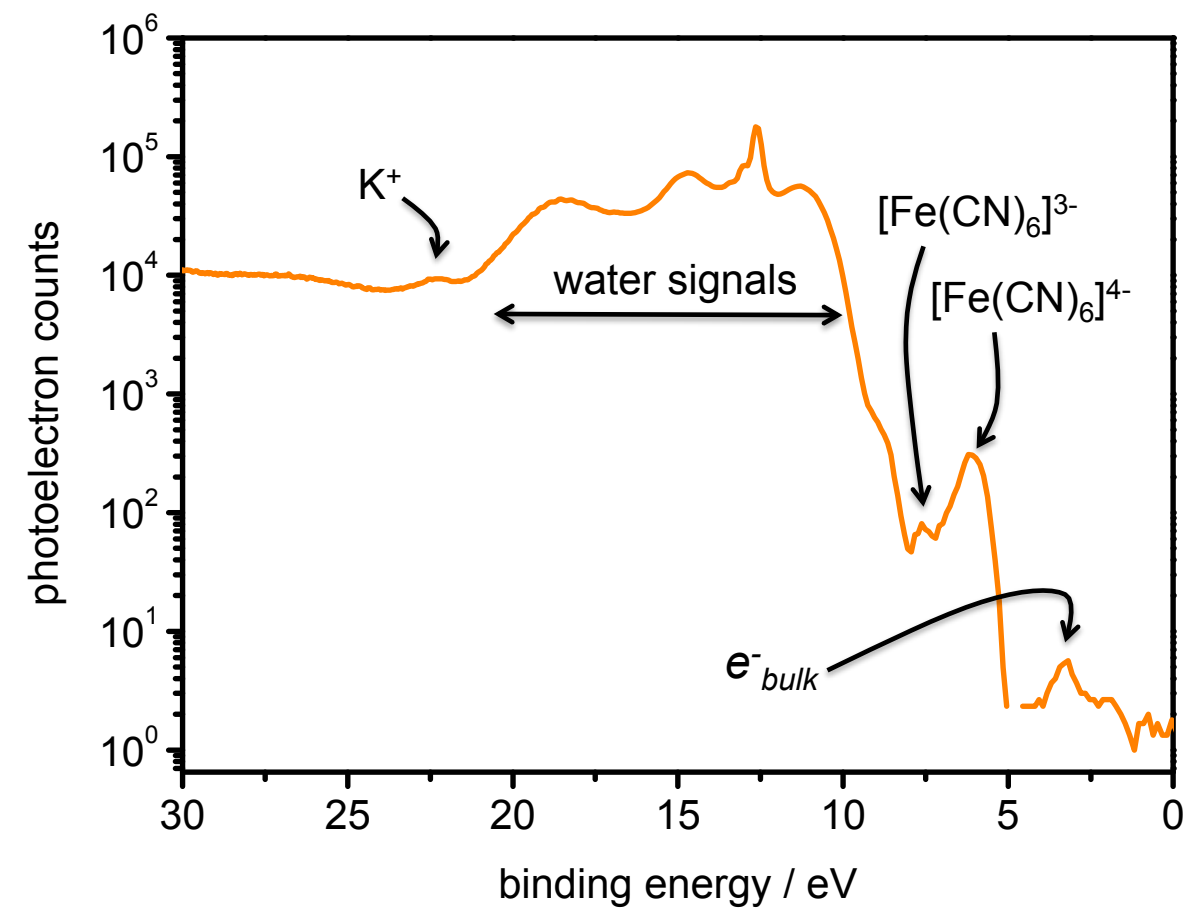

Fig. 3.10: Entire photoelectron spectrum with signals from the bulk solvated electron, the $\left[\mathrm{Fe}(\mathrm{CN})_{6}\right]_{(\mathrm{aq})}^{4-}\left(\mathrm{E}_{\mathrm{bind}}=6.2 \mathrm{eV}\right)$ as well as the corresponding oxidized complex $\left[\mathrm{Fe}(\mathrm{CN})_{6}\right]_{(\mathrm{aq})}^{3-}\left(\mathrm{E}_{\text {bind }}=7.6 \mathrm{eV}\right)$, the water signals between $9-20 \mathrm{eV}$, and a signal arising from potassium ions. The intensity scale is logarithmic in order to allow a direct comparison of signal intensities. 



\section{Discussion}

\subsection{Bulk solvated electrons}

We have determined a vertical binding energy of $3.3( \pm 0.1) \mathrm{eV}$ for the bulk solvated electron (see section 3.2). Since our experimental approach only allows us to detect photoelectrons from the first $\sim 6$ monolayers $(\approx 18 \AA)$ of the liquid, it is legitimate to ask if solvated electrons within this detection range can be considered as 'real' bulk solvated electrons. In an ab initio molecular dynamics study of the aqueous liquid-vapor interface, Kuo and Mundy [131] found that $10 \AA$ is the shortest distance from the interface at which one can observe bulk liquid behavior. We thus believe that our value of $3.3 \mathrm{eV}$ is at least very close to the binding energy of the bulk hydrated electron. Very recently, Tang et al. confirmed this result [149]. With a slightly different experimental approach they measure a binding energy of $3.27 \mathrm{eV}$ for the bulk solvated electron. Importantly, their photoelectrons possess kinetic energies of only $1-2 \mathrm{eV}$, which means that their attenuation length is significantly longer compared to our experiment (see section 2.3.4, [140]). Accordingly, photoelectron spectroscopy in their experiment is by far less sensitive to the surface.

Our measurement can be compared to a number of mixed quantum/classical simulations on hydrated electrons in bulk water and large water clusters [32,34,35]. Latest results on relaxed thermal nanoscale water cluster anions with up to 8000 water molecules reveal an electronic ground state energy of $-4.4 \mathrm{eV}$ for the bulk hydrated electron [35]. Previous results obtained with the same method neglected long range interactions and thus underestimated the ground state energy by about $1 \mathrm{eV}$ [85]. However, it is the question if the electronic ground state energy can be compared to experimentally determined values, which may comprise electron polarization effects during the photoemission process (see section 2.3.2).

Fig. 4.2 shows our measurement for the VBE of bulk and surface solvated electrons together with experimental results for anionic water clusters of different sizes $[26,28,71,72]$. For clusters, the term vertical detachment energy (VDE) is used to describe the vertical binding energy of an excess electron. The VDEs of anionic water clusters $\left(\mathrm{H}_{2} \mathrm{O}\right)_{\mathrm{n}}^{-}$are typically plotted versus $\mathrm{n}^{-1 / 3}$, which corresponds to the inverse cluster radius. Accordingly, $\mathrm{n}^{-1 / 3} \rightarrow 0$ marks the bulk limit. The figure 


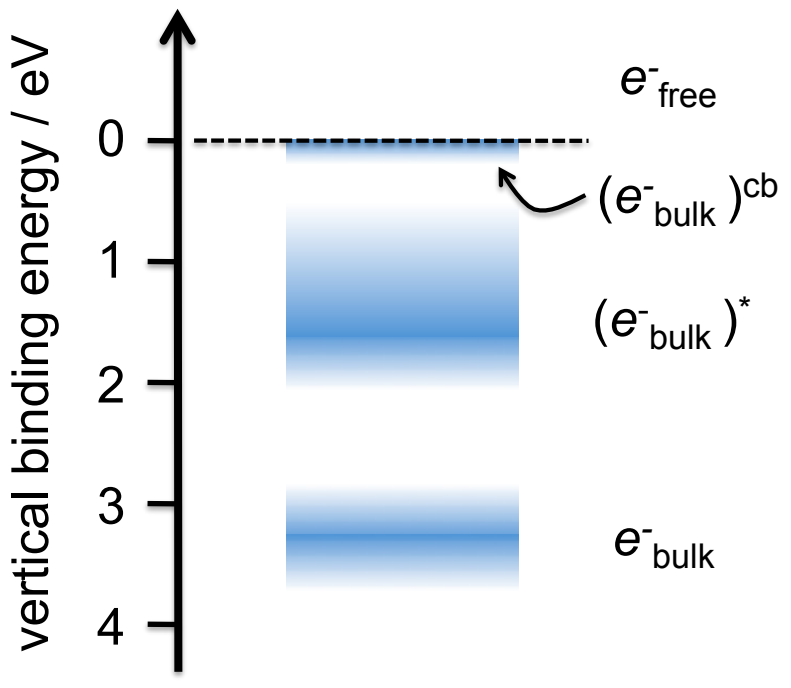

Fig. 4.1: Energy scale of the solvated electron in liquid water. Electronically excited states of the hydrated electron $\left(e_{\mathrm{bulk}}^{-}\right)^{*}$ possess a binding energy of $0.9-2 \mathrm{eV}$. The shaded area of $\left(e_{\text {bulk }}^{-}\right)^{*}$ approximates the shape of the $\left(e_{\text {bulk }}^{-}\right)$absorption spectrum with a maximum at $720 \mathrm{~nm}$. Stabilization of an electron in the conduction band $\left(e_{\text {bulk }}^{-}\right)^{c b}$ amounts between 0.12 and $0 \mathrm{eV}[24]$.

shows that our value of $3.3 \mathrm{eV}$ agrees with measurements for isomer $I$. This supports the recent interpretation, that for sizes $\mathrm{n} \geq \sim 50$ isomer $I$ represents clusters with an internally solvated electron [29,30].

Our result of $3.3 \mathrm{eV}$ sets the energy scale of the solvated electron in liquid water (Fig. 4.1). The electronically excited states $\left(e_{\text {bulk }}^{-}\right)^{*}$ of the hydrated electron are 3 non-degenerate $p$-type orbitals [40]. According to the absorption band of the hydrated electron with its maximum at $720 \mathrm{~nm}(1.7 \mathrm{eV})$, the electronically excited states lie between 1.3 and $2.4 \mathrm{eV}$ above the ground state [150]. The binding energy of an excited hydrated electron accordingly amounts to $0.9-2 \mathrm{eV}$. The nature and the energetics of the conduction band of liquid water are still subject to debate. An important quantity in this context is the solvent stabilization energy $V_{0}$. It describes how much solvent polarization stabilizes a conduction band electron $\left(e_{\text {bulk }}^{-}\right)^{c b}$ relative to the vacuum. The latest estimates suggest that $V_{0}$ amounts between -0.12 and $0 \mathrm{eV}[24,47]$. 


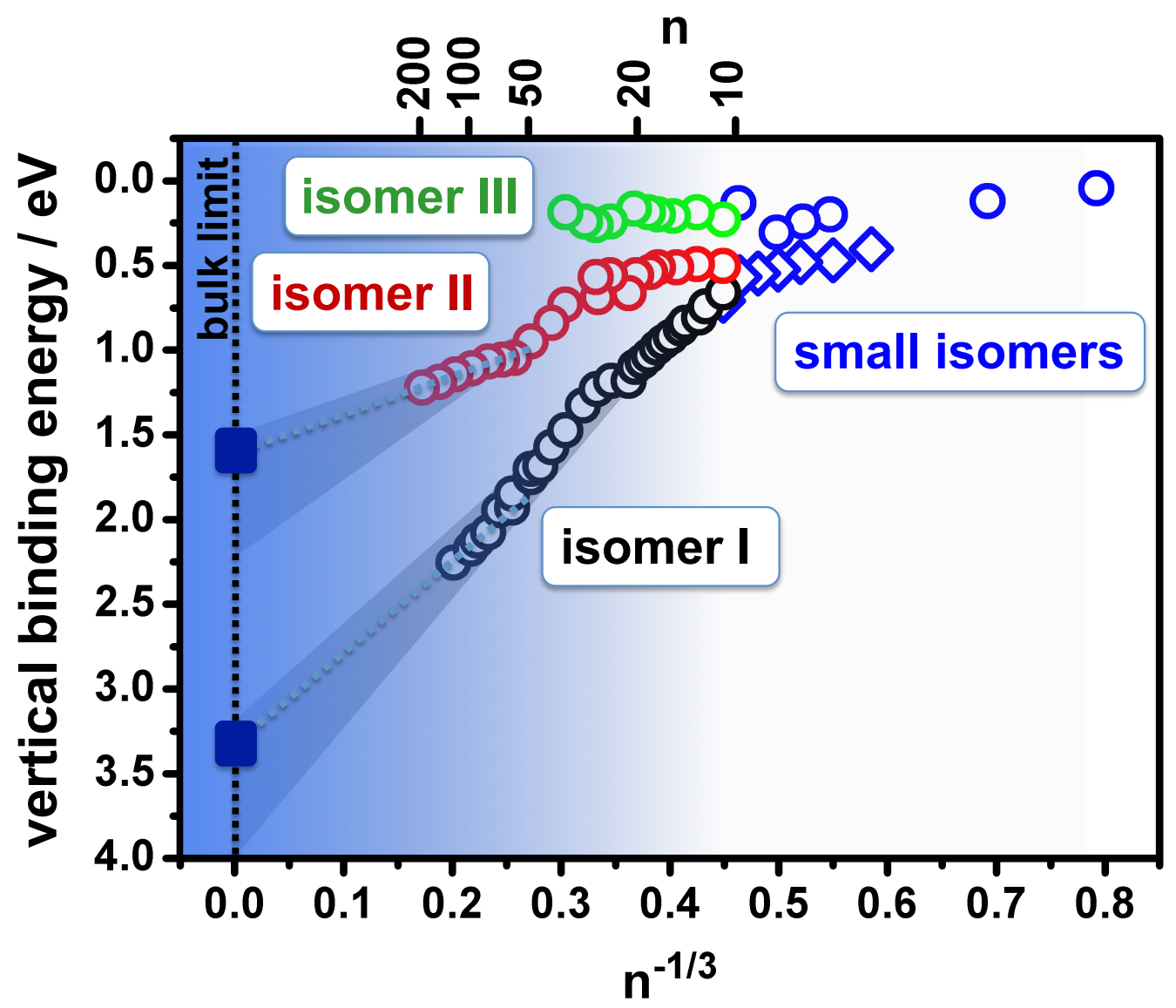

Fig. 4.2: Experimental VDEs for anionic water clusters $\left(\mathrm{H}_{2} \mathrm{O}\right)_{\mathrm{n}}^{-}$of various sizes $\mathrm{n}$, together with our results for the VBEs of bulk $(3.3 \mathrm{eV})$ and surface solvated $(1.6 \mathrm{eV})$ electrons (blue filled squares). Blue open circles and diamonds are data for small clusters from Johnson's group [71]. Black (isomers I), red (isomers $I I$ ) and green (isomers III) open circles are cluster data from Neumark's group [26,72]. The data from Bowen's group [28,70] are close to those of isomer I. Recent results from v. Issendorff's group [30] suggest that VDEs for internally solvated electrons $(\mathrm{n} \geq 50)$ are about $0.2 \mathrm{eV}$ higher than those of isomer $I$. The shaded area represents the uncertainty for an extrapolation of cluster values towards the bulk. The dotted lines interpolate between the largest clusters and our data. 


\subsection{Surface solvated electrons}

Our results prove the existence of electrons solvated on the surface of liquid water. Their vertical binding energy amounts to $1.6( \pm 0.1) \mathrm{eV}$ and their lifetime exceeds $100 \mathrm{ps}$ (see section 3.1). While the existence of such surface solvated electrons has already been postulated on the basis of theoretical investigations, their stability and lifetime revealed by our experiment is rather surprising. The apparent long lifetime indicates that the surface solvated electron survives many hydrogen bond rearrangements in the surrounding network of water molecules. From mixedquantum classical molecular dynamics simulations of excess electron relaxation dynamics at water/air interfaces and in large anionic water clusters [34,85], it was believed that electrons initially located on a liquid water surface dive into the bulk within a couple of picoseconds. In their simulations, Madarasz et al. found that $60 \%$ of initial surface solvated electrons ended up in interior solvated states after $10 \mathrm{ps}$. To some extend the discrepancy between these simulations and our experimental results might be due to the higher temperatures of $300 \mathrm{~K}$ in the simulations compared to about $273-278 \mathrm{~K}$ in our experiments. Indeed, experiments revealed that electron diffusion coefficients for $273-278 \mathrm{~K}$ are by more than a factor of two smaller compared to $300 \mathrm{~K}[151,152]$. In addition, a general change in the hydrated electron diffusion dynamics is observed below temperatures of $288 \mathrm{~K}\left(15^{\circ} \mathrm{C}\right)$. This result is supported by recent mixed quantum-classical molecular dynamics simulations [153].

Mixed-quantum classical molecular dynamics simulations of electrons at a supercooled liquid water/air interface at $200 \mathrm{~K}$ show that electrons remain in surface states for more than $180 \mathrm{ps}$ [85]. In these simulations the surface solvated electrons possess a ground state energy of about $-4 \mathrm{eV}$ which is close to the ground state energy of a bulk electron of $-4.4 \mathrm{eV}$ determined in the same study [34]. It is the question however, if those ground state energies can be directly compared to experimentally determined vertical binding energies (see section 4.1). In any case, the small difference in ground state energy between surface and bulk state is not in accord with our experimental findings.

Experiments and simulations of electron relaxation dynamics on the surface of ice show that electrons initially localize at pre-existing defects and subsequently induce dynamic reorientations of adjacent water molecules [86,89]. In a theoretical study, Hermann et al. [87] found the highest electron binding energy of $1.1 \mathrm{eV}$ for a surface vacancy site that provides several dangling $\mathrm{OH}$ bonds. In a combined experimental and theoretical approach, Bovensiepen et al. show that subsequent structural rearrangements of adjacent water molecules may further increases the binding energy by about $0.6 \mathrm{eV}$ [89]. Such a structural evolution is thermally 
activated and hence accelerated at increased temperatures. The electrons end up in surface cavities in which they can remain for several minutes [89].

From the lifetime of $e_{\text {surface }}^{-}$determined in our experiment, we propose a free energy barrier between surface and bulk solvated state (see Fig. 4.3). This barrier is attributed to the additional energy needed to form a bulk solvation pocket, which requires the concerted rearrangement of the subsurface molecules. It is likely that the stability of surface solvated electrons is further assisted by the specific arrangement of the solvating water molecules. Based on density functional theory (DFT) and molecular dynamics methods, Baletto et al. revealed the existence of a subsurface cavity just below an electron solvated on the surface of ice [86]. In contrast with the cavity of a bulk hydrated electron with $\mathrm{OH}$ bonds pointing towards the center, this cavity is mostly surrounded by oxygen atoms. This subsurface cavity may act as an electrostatic barrier which additionally prevents the penetration of the surface electron into the bulk.

It is enlightening to discuss the lifetime of surface solvated electrons in the context of solvated electron diffusion in water. For temperatures of $273-278 \mathrm{~K}$, the diffusion coefficient $D\left(e_{(\mathrm{aq})}^{-}\right)$amounts to $2.2 \cdot 10^{-5} \mathrm{~cm}^{2} / \mathrm{s}=0.22 \AA^{2} / \mathrm{ps}$ [151]. Assuming Brownian motion as a limiting case of the actual motion of the electron, the Einstein relation allows to determine the root mean square total displacement $\Delta r(t)$ between $t=0$ and $t[153]$ :

$$
\Delta r(t)=\sqrt{6 D\left(e_{(\mathrm{aq})}^{-}\right) t}
$$

For the present case and $t=100 \mathrm{ps}$, this gives $\Delta r=11.5 \AA \approx 4$ liquid water monolayers. Experiments on the temperature dependence of electron diffusion in water revealed a free energy of activation of $\mathrm{E}_{a}=0.21 \mathrm{eV}$ and Arrhenius behavior for temperatures between $15-90^{\circ} \mathrm{C}(288-363 \mathrm{~K})$ [151,152]. Although non-Arrhenius behavior is observed for temperatures $<15^{\circ} \mathrm{C}(288 \mathrm{~K})$ this value is certainly a good approximation. Taking these results, the mean total displacement and the connected energy of activation together, suggest that the free energy barrier between surface and bulk solvated states exceeds $0.2 \mathrm{eV}$, since the lifetime of the surface solvated electron is longer than 100 ps.

The activation energy on the other hand can be compared to the average energy required to break a hydrogen bond in liquid water. Smith et al. determined a value of $0.065 \mathrm{eV}$ for the energy required to break a hydrogen bond in liquid water while leaving the molecules essentially in the same position [154-156]. A barrier $\mathrm{E}_{a}>$ $0.2 \mathrm{eV}$ implies that the transfer of a surface solvated electron into the bulk requires the concerted breaking of more than 3 hydrogen bonds. 


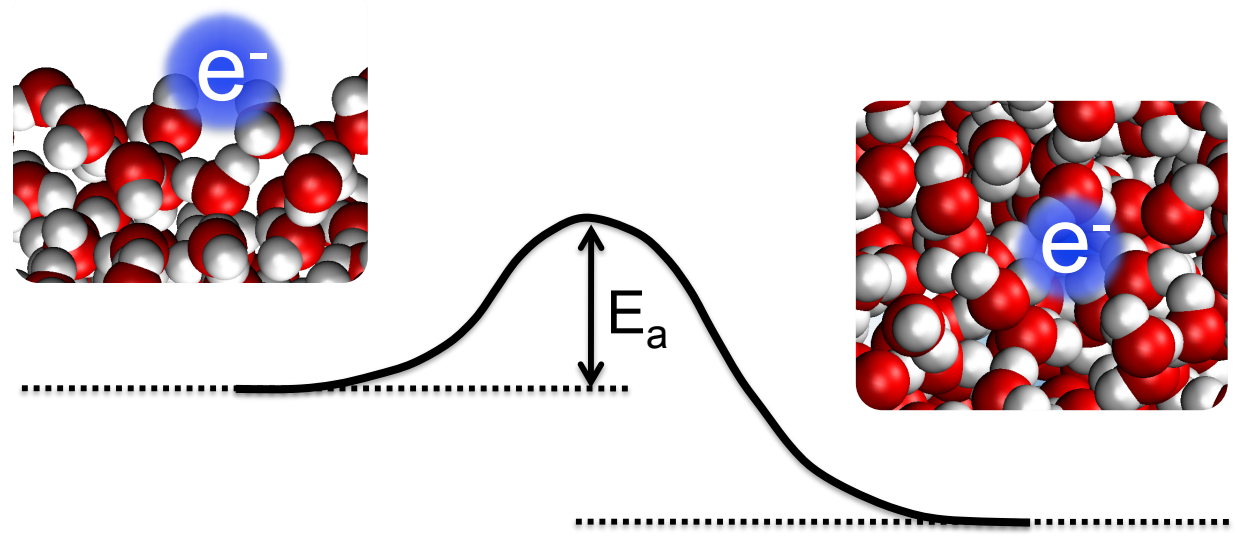

Fig. 4.3: From the lifetime of $e_{\text {surface }}^{-}$determined in our experiment we conclude on a free energy barrier between surface and bulk solvated state. The barrier may result from the (energetically unfavorable) formation of bulk solvation pockets, which involves the concerted making and breaking of many hydrogen bonds. It is likely that stabilization is assisted by an electrostatic barrier arising from the specific arrangements of the solvating water molecules.

Fig. 4.2 shows our values for the VBE of bulk and surface solvated electrons together with experimental results for anionic water clusters $\left(\mathrm{H}_{2} \mathrm{O}\right)_{\mathrm{n}}^{-}$of different sizes $\mathrm{n}[26,28,71,72]$. The figure shows that our value of $\operatorname{VBE}\left(e_{\text {surface }}^{-}\right)=1.6 \mathrm{eV}$ is in line with results for isomer $I I$ for cluster sizes $\mathrm{n} \geq 50$. This class is attributed to clusters with a surface solvated electron. Since electrons in clusters with $n \geq 50$ preferentially occupy internal states, surface states are considered as metastable [30] (see also section 1.2). Isomer III is a class of weakly bound surface electrons, which was only observed for $\left(\mathrm{D}_{2} \mathrm{O}\right)_{\mathrm{n}}^{-}$and cluster sizes $\mathrm{n}<50[26,72]$. When discussing results for anionic water clusters it is important to note that they exhibit a complex potential energy landscapes with multiple potential energy minima separated by significant barriers [75,79]. Accordingly, when multiple isomers are formed and investigated in an experiment, their population may be far out of thermal equilibrium $[29,30,35,36]$.

The existence of surface solvated electrons has important implications to many interfacial processes in physics, chemistry and biology. As outlined in sections 4.3.1 and 4.3.2, they may play an important role in DNA damage as well as in the context of the Antarctic ozone hole. Similarly, they may present a hitherto unrecognized reactive species on the surface of sea-water aerosol particles [157], on the seawater surface [158] and on liquid water interfaces in general. 


\subsection{Resonant dissociative electron attachment}

Our results define the energy scale of the solvated electron in liquid water and at liquid water interfaces, which is key to understanding of the mechanism of many electron transfer processes in nature. This section introduces the concept of Resonant Dissociative Electron Attachment (RDEA) of partially and fully solvated electrons to molecules in aqueous environments. The idea for RDEA is based on dissociative electron transfer and attachment experiments to molecules adsorbed on metal surfaces, polar ice surfaces and polar liquids [16, 17, 102]. Although experiments already suggested the existence of particular resonances for electron transfer and attachment, our results on the energy scale of hydrated electrons allows us, for the first time, to understand these processes with a simple and comprehensive picture.

In general, it is assumed that the process is initiated by the transfer of an electron into a low-lying, antibonding LUMO (lowest unoccupied molecular orbital), and the occupation of this orbital results in direct or indirect bond rupture. The first step in this process, electron attachment, depends upon the energetics of the antibonding LUMO of the acceptor molecule with respect to the energetics of the (hydrated) electron. In an aqueous environment the potential energy curves of neutral species and their corresponding anions are quite different from their curves in the gas phase (see also section 1.3). Fig. 4.4 depicts this situation for electron attachment to the cytosine $\pi^{*}$ orbital in a cytosine-sugar-phosphate DNA fragment. Characteristics of the curves are adopted from references $[9,10]$. The figure shows the energies of the neutral and attached anionic state as a function of the sugar-phosphate $\mathrm{C}-\mathrm{O}$ bond length in the absence of any solvation (left) and with solvation (right). The same qualitative picture can be expected for electron attachment to any DNA base $\pi^{*}$ orbital $[93,94,97]$ as well as electron attachment to chlorofluorocarbons (CFCs) $[16,17,102]$ and presumably many other molecular systems.

The three following points highlight the main differences between the two situations in Fig. 4.4 and thus the impact of solvation in electron attachment:

(1) When an electron attaches to a neutral species in the gas phase, this electron attachment generally requires a certain amount of energy (except for the formation of dipole-bound states). The required energy corresponds to the difference in energy between the neutral and anionic curve at the equilibrium bond length of the neutral curve (= internuclear distance at the minimum of the neutral potential energy curve). Accordingly, the attaching electron has to possess a 'matching' kinetic energy in order to successfully attach to the molecule and to form a so-called shape resonance anion. This picture is consistent with experimental findings on electron attachment to dry DNA (see section 1.3) and other molecular systems. 

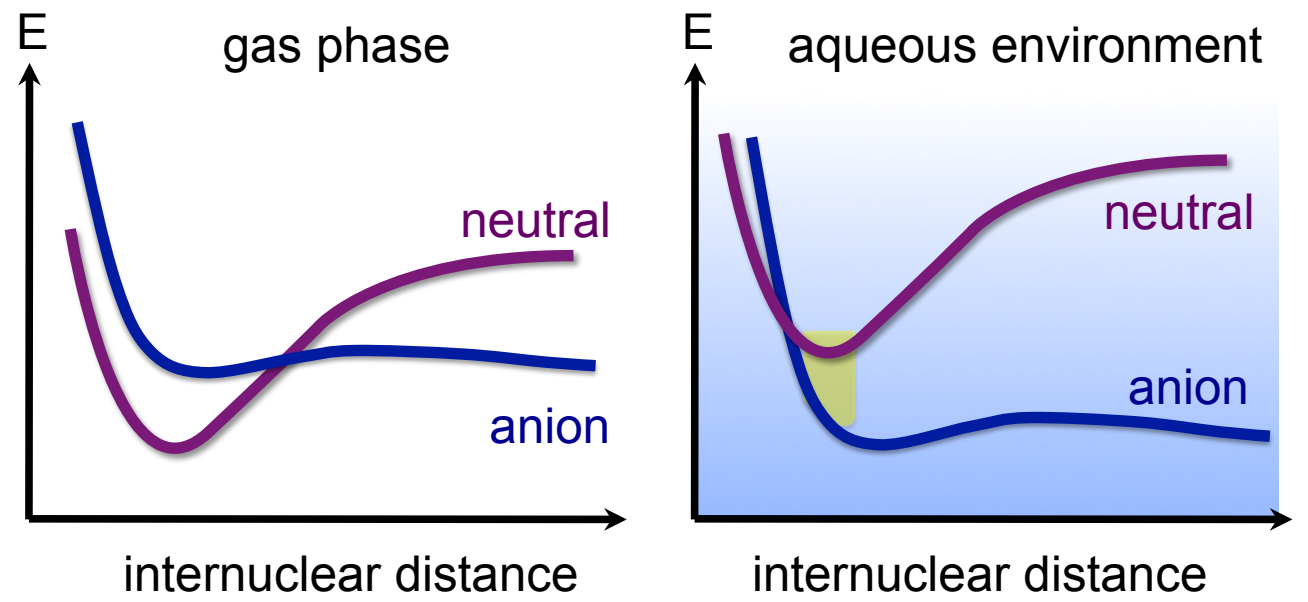

Fig. 4.4: The figure shows the impact of solvation on the energies of the neutral and attached anionic state as a function of the internuclear distance in a chemical bond. The curves are adopted from references $[9,10]$ and represent the situation for electron attachment to the cytosine $\pi^{*}$ orbital in a cytosine-sugar-phosphate DNA fragment. The same qualitative picture can be expected for electron attachment to any DNA base $\pi^{*}$ orbital and presumably many other molecular systems.

(2) Breaking covalent bonds in a neutral species requires a significant amount of energy (ca. $4 \mathrm{eV}$ in DNA), as indicated by the increase of the neutral potential energy curves with increasing internuclear distance in Fig. 4.4. This situation is different after electron attachment to an antibonding orbital. For a bond break in the anionic species, comparatively low energy barriers have to be surmounted (anionic curves in Fig. 4.4) [9]. In the gas phase, however, these small barriers are the reason why the majority of the shape resonance anions undergo autodetachment, and thus return to the neutral state [9].

(3) The situation in an aqueous environment is much different from the picture in the gas phase. Although the shape of the curves does not change explicitly with solvation [10], the interactions with polar solvent molecules stabilize the anion. As a result, the potential energy curve of the anion lies below the corresponding neutral curve (see right part of Fig. 4.4) [10]. This has far reaching consequences on electron attachment in aqueous environments. On the one hand, this means that bond cleavage does not compete with autodetachment, since this would require a significant amount of energy [10]. On the other hand, and much more important is, 
that electron attachment is only possible in the region of overlap of the neutral and anionic curves. This overlap can be considered as an electron acceptance window and is indicated by the yellow area in Fig. 4.4. Since this window represents the overlap of the wave-functions of neutral and anionic species, it is obviously related to a Franck-Condon overlap.

The key point in the RDEA concept is the comparison of this electron acceptance window - that is resonances for electron transfer and attachment - of molecules in aqueous environments with the energy scale of the hydrated electron. The following sections demonstrate the importance of RDEA for DNA damage and atmospheric chemistry.

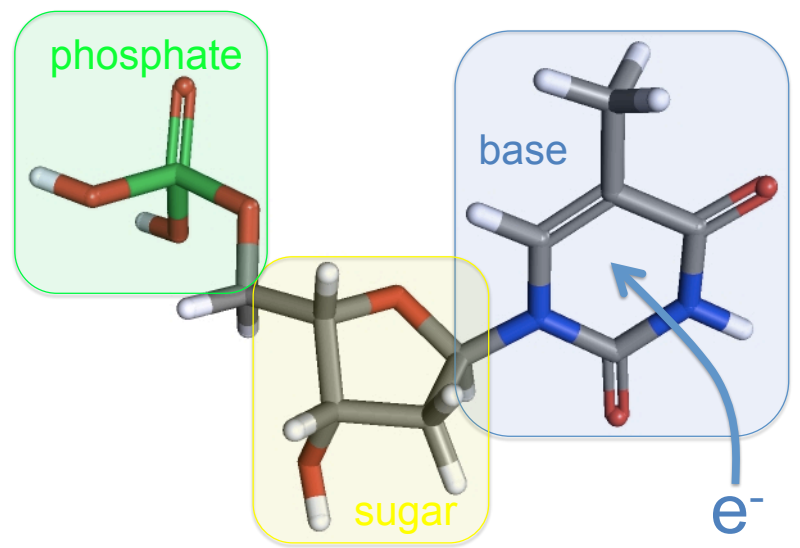

Fig. 4.5: Electron attachment to the thymine base in 2'-deoxythymidine-5'monophosphate (5'-dTMPH). Subsequently to electron attachment, a glycosidic bond break between the base and the sugar unit, or a C-O bond break between the sugar and the phosphate unit can occur [97].

\subsubsection{RDEA to DNA in aqueous environments}

In order to apply the RDEA concept to DNA, one has to determine the electron acceptance windows of DNA and compare them to the energy scale of the hydrated electron. In the following we only consider electron attachment to DNA bases within a DNA fragment, but we propose a general way to determine electron acceptance windows for any species in aqueous solution. We find that knowledge of three quantities is required to determine the electron acceptance windows of a system under investigation: the vertical electron affinity (VEA), the adiabatic electron affinity (AEA) and the vertical detachment energy (VDE) of the anion. Fig. 4.7 clarifies the importance of these quantities: 
$\mathrm{VEA}=\mathrm{E}($ optimized neutral system $)-\mathrm{E}$ (anion at optimized neutral geometry) The vertical electron affinity is the difference in energy between the neutral and anionic species, both with the optimized geometric structure and solvation structure of the neutral species.

$\mathrm{AEA}=\mathrm{E}($ optimized neutral system $)-\mathrm{E}($ optimized anion $)$

The adiabatic electron affinity is the difference in energy between neutral and anionic species, whereas both are in their own optimized solvation environment and in their optimized geometric structure.

$\mathrm{VDE}=\mathrm{E}$ (neutral system at optimized anion geometry) - E(optimized anion) The vertical detachment energy is the difference in energy between the neutral and anionic species, both with the optimized geometric structure and solvation structure of the anionic species. In other words, the VDE is the energy required to remove the electron from the anionic state, without changing the initial geometry of the system.

These quantities define the upper and lower limit of the electron acceptance window. It is important to note that both values are electron binding energies as indicated in Fig. 4.7.

upper limit $=\mid$ VDE $|-|$ AEA $\mid$

lower limit $=\mid$ VDE $|-|$ AEA $|+|$ VEA $\mid$

$\mathrm{Gu}$ et al. theoretically determined the required quantities for the bases thymine and cytosine in various DNA fragments [93-96,98]. A complete set of data is available for 2'-deoxythymidine-5'-monophosphate (5'-dTMPH) and 2'-deoxycytidine-3'monophosphate (3'-dCMPH) (see following tables). Figures 4.5 and 4.6 depict the situations for 5'-dTMPH and 3'-dCMPH, respectively.

2'-deoxythymidine-5'-monophosphate (5'-dTMPH) [94]

\begin{tabular}{llll}
\hline VEA & AEA & VDE & electron acceptance window \\
\hline $1.5 \mathrm{eV}$ & $2.0 \mathrm{eV}$ & $2.5 \mathrm{eV}$ & $0.5-2.0 \mathrm{eV}$ \\
\hline
\end{tabular}

2'-deoxycytidine-3'-monophosphate (3'-dCMPH) [93]

\begin{tabular}{llll}
\hline VEA & AEA & VDE & electron acceptance window \\
\hline $1.7 \mathrm{eV}$ & $2.2 \mathrm{eV}$ & $3.0 \mathrm{eV}$ & $0.8-2.5 \mathrm{eV}$ \\
\hline
\end{tabular}




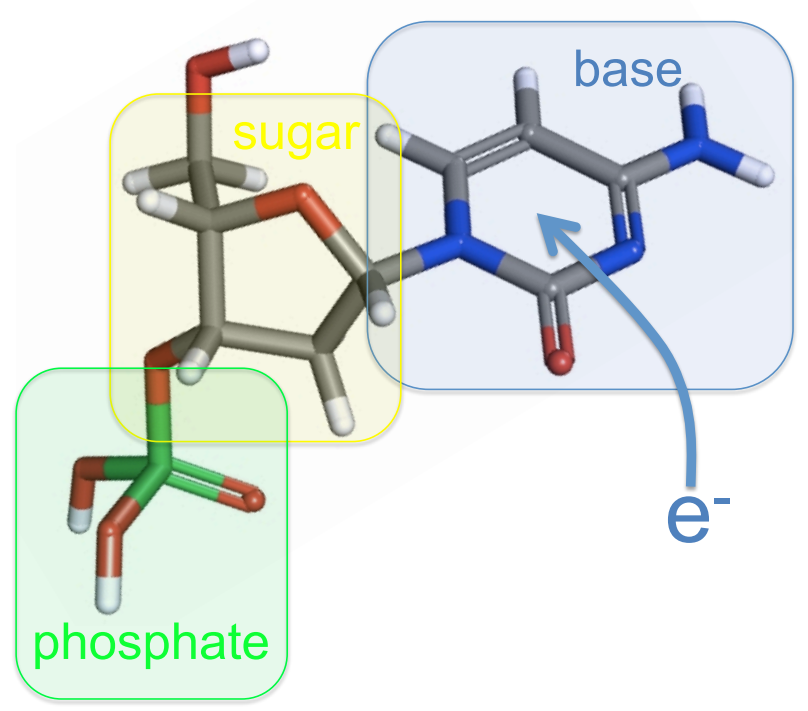

Fig. 4.6: Electron attachment to the cytosine base in 2'-deoxycytidine-3'-monophosphate (3'-dCMPH). Subsequently to electron attachment, a glycosidic bond break between the base and the sugar unit, or a $\mathrm{C}-\mathrm{O}$ bond break between the sugar and the phosphate unit can occur.

Interestingly, $\mathrm{Gu}$ et al. found that in aqueous solution, deprotonation of the phosphate group resulting in $5^{\prime}-\mathrm{dTMP}^{-}$and $3^{\prime}-\mathrm{dCMP}^{-}$, respectively, does not have a significant influence $(\leq 0.1 \mathrm{eV})$ on the VEA, AEA and VDE values. This similarity suggests that the quantities are independent of the counterion in aqueous solution [93, 94]. For electron attachment to the thymine base in larger DNA fragments, such as dApdT, the AEA amounts to $2.16 \mathrm{eV}$ which is slightly larger compared to about $2.0 \mathrm{eV}$ in 5'-dTMPH. This may indicate a slight increase in electron-capturing ability for larger DNA fragments [98].

Fig. 4.7 illustrates the RDEA situation for electron attachment to 5'-dTMPH and directly shows the importance of the RDEA concept. The electron acceptance window is indicated by the white band and can be directly compared to the energetics of the hydrated electron shown on the left side of the figure. The striking point here is that partially hydrated electrons, that is electrons bound to interfaces as well as excited hydrated electrons, are in resonance with the electron acceptance windows of DNA bases. Completely hydrated electrons, however, are almost out of the resonance and therefore a very slowly reacting species in this context. The picture is supported by the recent experimental finding by Wang et al. that pre-hydrated electrons rapidly attach to DNA bases $[7,8]$. 


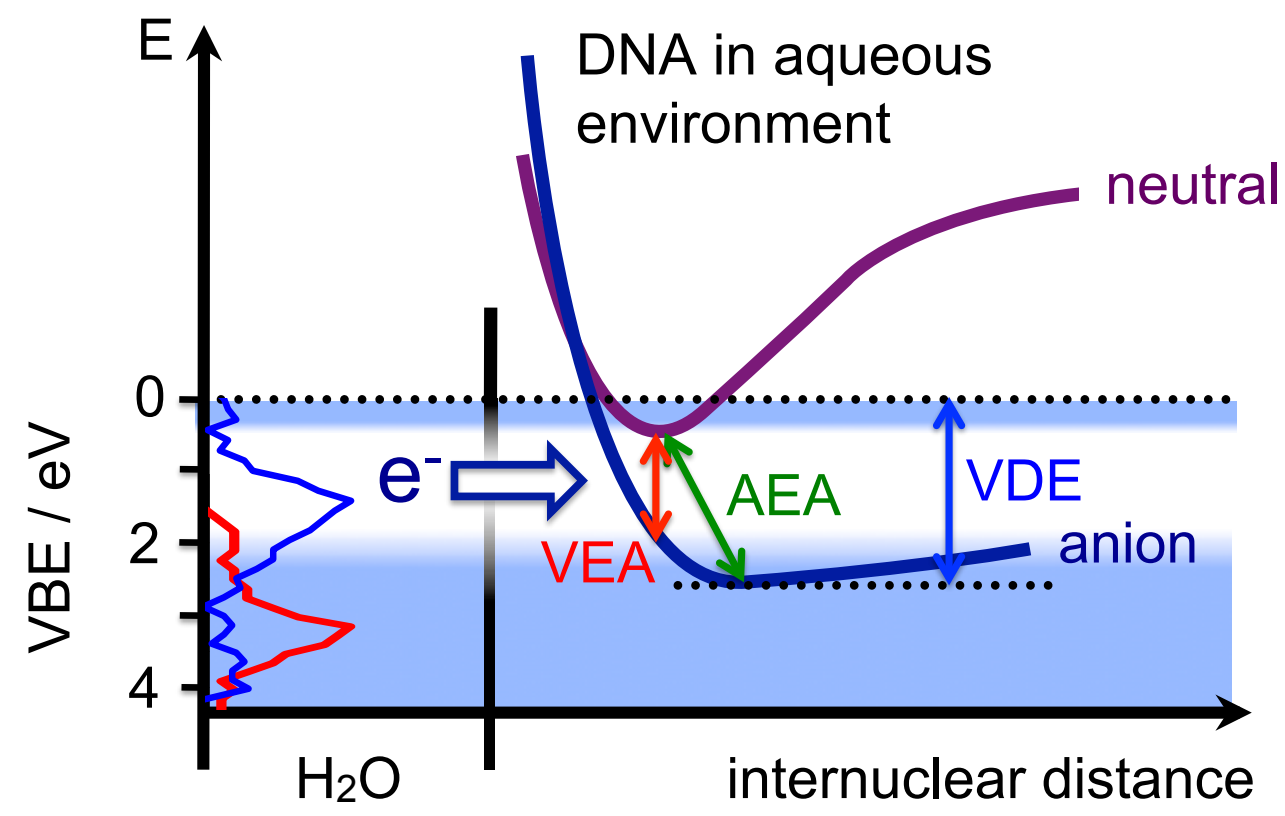

Fig. 4.7: The figure illustrates the RDEA concept for electron attachment to the thymine base in 5'-dTMPH. The electron acceptance window is indicated by the white band and can be directly compared to the energetics of hydrated electrons (red) and interface/partially hydrated electrons (blue) on the left side of the figure. Importantly, the latter are in resonance with the electron acceptance windows of the DNA base. Similar situations are expected for electron attachment to other DNA bases. Subsequently to electron attachment, a glycosidic bond break between the base and the sugar unit, or a C-O bond break between the sugar and the phosphate unit can occur. The internuclear distance represents respective $\mathrm{C}-\mathrm{N}$ and $\mathrm{C}-\mathrm{O}$ bond distances.

Importantly, the RDEA picture also indicates that an electron has to possess a certain matching binding energy in order to successfully attach to a DNA base. This means that electrons with higher energy, that is with binding energies between $0 \mathrm{eV}$ and the onset of the electron acceptance window and even free electrons (see Fig. 4.1) are not reactive in this context. In aqueous solution, initially free electrons are rapidly slowed down by collisions and stepwise hydrated by water molecules, finally resulting in a hydrated electron. Since the hydration process proceeds within about a picosecond (max. 5 ps [47]), this does not leave the electron much time to resonantly attach to any species. In other words, the time span in which the electron possesses a matching binding energy is very short $(<1 \mathrm{ps})$. It is worthwhile to speculate whether electrons bound to interfaces between water and (hydrophobic) biomolecules have energetic properties similar to the surface 
bound electrons revealed in our experiment. Such interfaces may temporarily trap electrons at binding energies resonant with DNA bases, extend their lifetime and thus enhance their possibility to resonantly attach.

The RDEA concept may have far reaching consequences for our understanding of radiation-induced DNA damage, since it suggests that the class of partially hydrated electrons is responsible for a large portion of it $[7,8]$. It also points the way for further experimental and theoretical studies with the goal to quantitatively understanding electron attachment to different DNA bases and the subsequent reaction paths.

\subsubsection{Implications for atmospheric chemistry}

Knowledge on the energy scale of bulk and surface solvated electrons also sheds light on the dissociative electron attachment to chlorine-containing molecules on the surface of atmospheric ice particles. This scenario might be of significant importance to the formation of the Antarctic ozone hole (see section 1.4). In the following we assume that the binding energy of electrons on the surface of ice is very similar to the binding energy for surface solvated electrons revealed by our experiments. Indeed, sum-frequency vibrational spectroscopy measurements on the surface of ice indicate a disordering and the formation of a quasi-liquid layer on the surface for temperatures $\mathrm{T} \geq \sim 200 \mathrm{~K}$ [159].

Fig. 4.8 schematically depicts the RDEA situation representative for CFCs - and presumably many similar Cl-, Br- and I- containing molecules - adsorbed on a water ice surface $[16,17]$. The right-hand side shows the potential energy curves of the neutral and anionic states. From experimental data and estimates on the impact of a water ice surface on the potential energy curves of adsorbed molecules, $\mathrm{Lu}$ et al. concluded that the electron acceptance window for CFCs ranges from about 1 to $1.5 \mathrm{eV}$ binding energy [16,17]. The left part of the Fig. 4.8 compares the acceptance window - represented by the white band - with the binding energies of surface and bulk hydrated electrons. The significant overlap of the white band with the photoelectron spectrum of the surface electrons (shown in blue) indicates that surface electrons are in resonance with the electron acceptance window of the molecule. They possess the correct binding energy to enter the antibonding LUMO of the molecule and to induce a bond break which results in the release of a $\mathrm{Cl}^{-}$. The long lifetimes of electrons bound to the surface of ice range up to minutes [89], which helps facilitate electron attachment [18]. It is important to note that, while the overlap with bulk hydrated electrons (red curve in Fig. 4.8) is negligible, there is a strong overlap with excited states of the bulk hydrated electron (see Fig. 4.1). 


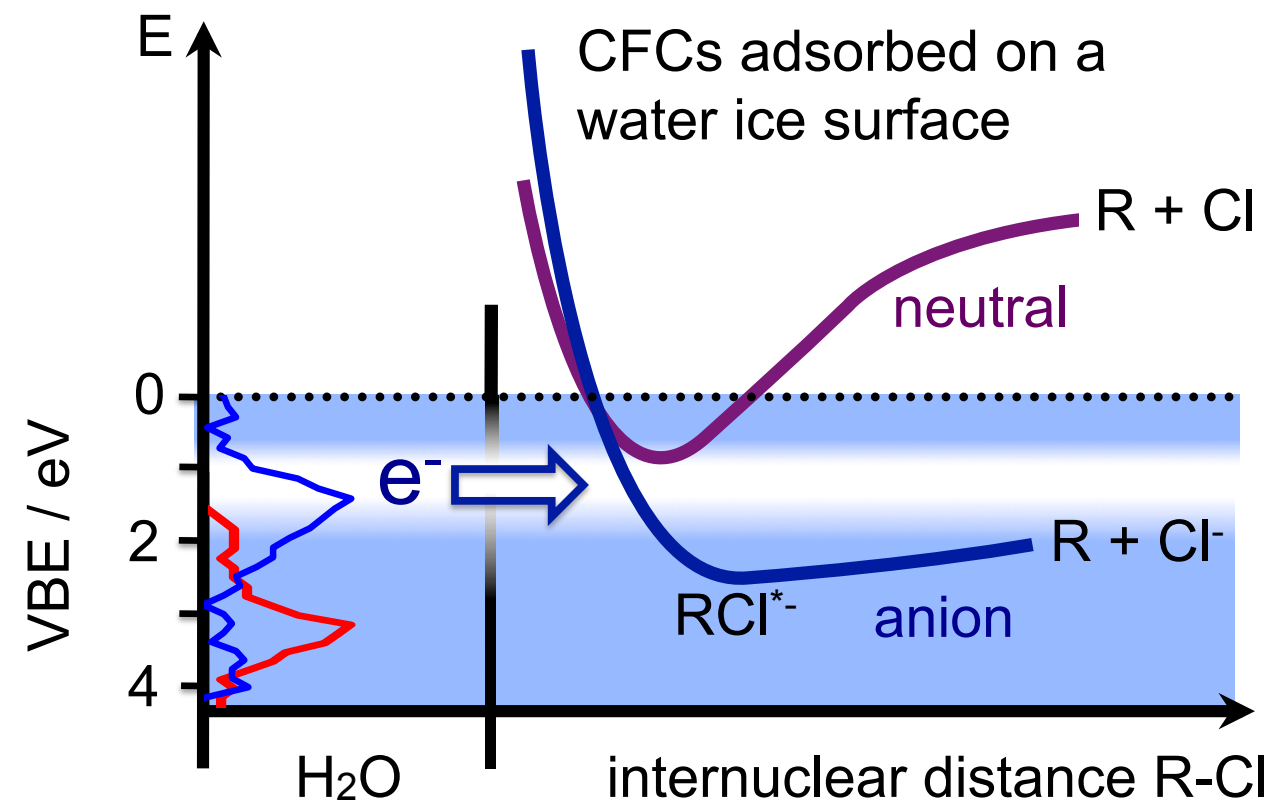

Fig. 4.8: The figure illustrates the RDEA concept for electron attachment to CFCs adsorbed on a water ice surface. The electron acceptance window ranges from about 1 to $1.5 \mathrm{eV}$ binding energy [16,17]. It is indicated by the white band and can be directly compared to the energetics of solvated electrons (red) and interface/partially solvated electrons (blue) on the left side of the figure. Importantly, the latter are in resonance with the electron acceptance windows. Similar situations might be expected for many other Cl-, Br- and I- containing molecules.

However, due to their fast relaxation - within a few picoseconds - they do not seem to play a significant role in the present case.

The matching binding energy of the surface solvated electron nicely explains the high dissociative electron attachment cross sections for CFCs and presumably many other Cl-, Br- and I- containing molecules adsorbed to water ice surfaces. While this step now seems to be well understood, the question about how the $\mathrm{Cl}^{-}$is subsequently converted into the harmful $\mathrm{Cl}$ species remains unanswered. Since it is rather unlikely that the $\mathrm{Cl}^{-}$desorbs from the atmospheric ice particle, reactions with other atmospheric molecules on the surface of the ice particle were proposed to convert the $\mathrm{Cl}^{-}$into $\mathrm{Cl}$. These involve the reactions with $\mathrm{ClONO}_{2}, \mathrm{~N}_{2} \mathrm{O}_{5}$ or $\mathrm{HNO}_{3}$ and convert $\mathrm{Cl}^{-}$into photoactive $\mathrm{Cl}_{2}, \mathrm{ClNO}_{2}$ or $\mathrm{HCl}$, respectively $[103,160]$.

Here, we present a new reaction sequence which might play a significant role in the conversion of $\mathrm{Cl}^{-}$into $\mathrm{Cl}$. Ionization of water by cosmic rays results in initially 
free electrons which are eventually trapped as a hydrated electron inside $e_{\text {bulk }}^{-}$or on the surface $e_{\text {surface }}^{-}$of the ice particle. However, ionization of a water molecule also leaves a cationic species behind, $\mathrm{H}_{2} \mathrm{O}^{+}$, which rapidly transfers $\mathrm{H}^{+}$to a neighboring water molecule, resulting in $\mathrm{H}_{3} \mathrm{O}^{+}$and $\mathrm{OH}$. In the following, note that all reactions (4.2 to 4.7) take place inside or on the surface of a water ice particle.

$$
\mathrm{H}_{2} \mathrm{O}+\text { cosmic rays } \longrightarrow e^{-}+\mathrm{H}_{3} \mathrm{O}^{+}+\mathrm{OH}
$$

A surface electron resonantly attaches to a chlorine-containing molecule adsorbed on the water ice surface. Since the probability for autodetachment of the $\mathrm{RCl}^{*-}$ species and thus a reverse reaction is low [17], the majority of electron attachment events is reactive:

$$
e_{\text {surface }}^{-}+\mathrm{RCl}_{(\text {surface) }} \longrightarrow \mathrm{RCl}_{(\text {surface })}^{*-} \longrightarrow \mathrm{Cl}_{(\text {surface })}^{-}+\mathrm{R}_{(\text {surface })}
$$

We suggest that the $\mathrm{Cl}^{-}$subsequently reacts with the $\mathrm{OH}$ formed in reaction 4.2. This reaction is known to take place in the bulk and at water interfaces [157]. Our assumption is further supported by the experimental and theoretical finding that chloride ions strongly attract surrounding reactive species, such as $\mathrm{OH}$ [157]:

$$
\mathrm{OH}+\mathrm{Cl}^{-} \longrightarrow\left(\mathrm{OH} \ldots \mathrm{Cl}^{-}\right)
$$

The structure of the bound complex $\left(\mathrm{OH} \ldots \mathrm{Cl}^{-}\right)$probably involves interaction between the hydrogen of the $\mathrm{OH}$ and the chloride ion $[157,161]$. The complex can subsequently either undergo a self-reaction or react with the remaining $\mathrm{H}^{+}$from reaction 4.2 :

$$
\begin{gathered}
\left(\mathrm{OH} . . \mathrm{Cl}^{-}\right)+\left(\mathrm{OH} . . . \mathrm{Cl}^{-}\right) \longrightarrow \mathrm{Cl}_{2}+2 \mathrm{OH}^{-} \\
\left(\mathrm{OH} \ldots \mathrm{Cl}^{-}\right)+\mathrm{H}^{+} \longrightarrow \mathrm{Cl}+\mathrm{H}_{2} \mathrm{O}
\end{gathered}
$$

An important point here is that by taking the reaction steps 4.2 to 4.4 and 4.5 or 4.6 together, one formally obtains:

$$
\mathrm{RCl}_{(\text {surface })}+\text { cosmic rays } \longrightarrow \mathrm{Cl} / \mathrm{Cl}_{2}+\mathrm{R}
$$

That is, the ice particle serves as a catalyst, which temporarily provides electrons for the splitting of CFCs - and presumably many other similar compounds - 
adsorbed to its surface. However, it is important to note that the above reactions are temperature dependent and might proceed slow at temperatures of the polar stratospheric ice particles of about $150-200 \mathrm{~K}$. In addition, it might also be that the reaction partners $\mathrm{OH}$ and $\mathrm{H}^{+}$are spatially separated from the $\mathrm{Cl}^{-}$and rather immobile due to the low temperatures. In this case, only the melting of the ice particles in the Antarctic spring would allow a reaction. A different possibility is that the $\mathrm{Cl}^{-}$from reaction step 4.3 remains until cosmic rays ionize a neighboring water molecule and thus provide the reaction partners $\mathrm{OH}$ and $\mathrm{H}^{+}$for the subsequent steps.

Taken together, the RDEA picture and the reaction sequence presented above, will certainly stimulate further experimental investigations and enrich discussions on the relevance of hydrated electrons in Antarctic ozone hole formation and atmospheric chemistry in general. 


\section{Outlook}

The results and their implications presented in this thesis set the stage for further studies dedicated to partially and fully solvated electrons in water. The binding energy values of $3.3 \mathrm{eV}$ for the bulk and $1.6 \mathrm{eV}$ for the surface solvated electron present important benchmark data for subsequent theoretical studies. In addition, the stability and long lifetime of $>100 \mathrm{ps}$ of the surface solvated electron is an important information in this regard. As outlined in section 4.2 , the fact that recent theoretical predictions are not in agreement with the stability of surface solvated electrons revealed by our experiment may also be a matter of temperature. Further experimental and theoretical investigations could resolve this discrepancy and enrich our understanding on the interaction of charged species with water - or with a hydrogen bond network in general.

The surface solvated electron will certainly stimulate further experimental investigations. Of particular interest is a precise value for the lifetime of surface solvated electrons, as well as further information on the free energy barrier between bulk and surface states. Temperature-dependent measurements of the lifetime could allow valuable insights in this regard (section 4.2), and our experimental setup is particularly suited to access this kind of information. However, these measurements demand further improvement of the overall signal count rate. A recent improvement of our high harmonic light source is the first step in this direction. As a supplement, different experimental approaches may also be well suited to access this important information. Very recently, Verlet's group investigated the dynamics of hydrated electrons at the water/air interface [162]. They were using time-resolved second-harmonic generation (SHG) spectroscopy, a technique which is particularly sensitive to the surface. Their experimental strategy involved the generation of electrons via the charge-transfer-to-solvent (CTTS) transitions in iodide. Because iodide is particularly surface active, they observe the formation of bulk-like electrons near the surface, which are different from the surface solvated electrons detected in our experiment. Micro jet experiments using CTTS from Ior SHG experiments following two-photon ionization of pure water might resolve this discrepancy.

Our results on the energy scale of the hydrated electron together with the Resonant Dissociative Electron Attachment (RDEA) concept may have far-reaching conse- 
quences for our understanding of radiation-induced DNA damage (section 4.3.1). On the one hand, the concept suggests that excited hydrated electrons possess a particularly high potential for DNA damage. This is because their binding energy is in resonance with the electron acceptance windows of DNA bases. This statement is supported by recent experiments by Wang et al. [8]. Their experiments also reveal that purine bases (guanine $\mathrm{G}$, and adenine $\mathrm{A}$ ) are more efficient in capturing excited hydrated electrons than the pyrimidine bases (thymine $\mathrm{T}$, and cytosine C). Here, it would be particularly enlightening to compare experimental results with the theoretical predictions on the electron acceptance windows of the four DNA bases. Until now, sufficient theoretical data is only available for cytosine and thymine bases in small DNA units [93-96,98].

On the other hand, electrons solvated at interfaces between water and (hydrophobic) biomolecules may have energetic properties similar to the surface electrons revealed in our experiment. They might accordingly play an important role in DNA damage (section 4.3.1), and definitely deserve further experimental and theoretical investigations. Barbara's group already performed pump probe spectroscopy experiments on hydrated electrons in reverse micelles [163]. Similar experiments with probe wavelengths near the predicted absorption of surface solvated electrons of $1000-1200 \mathrm{~nm}$ [164] or with a continuum probe pulse could be promising starting points. To directly elucidate the importance of interfacial electrons for DNA damage, experiments such as the one presented by Wang et al. [8] (see above), could be performed in the presence of interfaces. Suitable interfaces could be the ones formed in emulsions of water with hydrophobic molecules or those formed by lipid-like molecules.

In atmospheric chemistry, the RDEA concept together with the reaction sequence, which we propose for the conversion of $\mathrm{Cl}^{-}$into harmful $\mathrm{Cl}$ (section 4.3.2), will certainly stimulate further experimental and theoretical investigations. In order to prove the proposed mechanism, experiments on large water clusters or ice surfaces present a promising approach. Here, adsorption of chlorine-containing molecules to the water surface and subsequent ionization of water molecules with UV light induces the proposed reaction sequence. The final product $\mathrm{Cl}$ can be detected via resonant multi-photon ionization (REMPI) [160]. An important feature of this system is the small UV absorption cross section of CFCs [165], which should guarantee that there are no contributions from direct photodissociation of the CFCs themselves.

The RDEA concept presented in this thesis not only allows promising new insights into radiation induced DNA damage and ozone depletion mechanisms, but also processes involving electrons in aqueous environments in general. It might, for 
example, also shed new light on the nature of electron transfer processes in aqueous solution, such as aqueous tunneling pathways between electron-transfer proteins [20]. Taken together, this work will certainly stimulate further experimental and theoretical investigations on the nature and implications of the hydrated electron. 



\section{Bibliography}

[1] Kevan, L (1981) Solvated electron structure in glassy matrices. Acc. Chem. Res. 14:138 Kevan structure of hydrated electron.

[2] Shkrob, IA, Glover, WJ, Larsen, RE, Schwartz, BJ (2007) The structure of the hydrated electron. part 2. a mixed quantum/classical molecular dynamics embedded cluster density functional theory: Single excitation configuration interaction study. J. Phys. Chem. A 111:5232.

[3] Hart, EJ, Boag, JW (1962) Absorption spectrum of hydrated electron in water and in aqueous solutions. J. Am. Chem. Soc. 84:4090.

[4] Boag, JW, Hart, EJ (1963) Absorption spectra in irradiated water and some solutions - absorption spectra of hydrated electron. Nature 197:45.

[5] Matheson, MS, Mulac, WA, Rabani, J (1963) Formation of hydrated electron in flash photolysis of aqueous solutions. J. Phys. Chem. 67:2613.

[6] Boudaiffa, B, P.Cloutier, Hunting, D, Huels, MA, L.Sanche (2000) Resonant formation of DNA strand breaks by low-energy (3 to $20 \mathrm{eV}$ ) electrons. Science $287: 1658$.

[7] Sanche, L (2009) Beyond radical thinking. Nature 461:358-359.

[8] Wang, CR, Ngugen, J, Lu, QB (2009) Bond breaks of nucleotides by dissociative electron transfer of nonequilibrium prehydrated elektrons: A new moleular mechanism for reductive DNA damage. J. Am. Chem. Soc. 131:11320.

[9] Simons, J (2006) How do low-energy (0.1-2 eV) electrons cause DNA-strand breaks? Acc. Chem. Res. 39:772.

[10] Barrios, R, Skurski, P, Simons, J (2002) Mechanism for damage to DNA by low-energy electrons. J. Phys. Chem. B 106:7991.

[11] Crespo-Hernandez, CE, Flores, S, Torres, C, Negron-Encarnacion, I, Arce, R (2000) Part I. Photochemical and photophysical studies of guanine derivatives: intermediates contributing to its photodestruction mechanism in aqueous solution and the participation of the electron adduct. Photochem. Photobiol. $71: 534$. 
[12] Siefermann, KR et al. (2010) Binding energies, lifetimes and implications of bulk and interface solvated electrons in water. Nat. Chem. 2:274.

[13] Brasseur, GP, Orlando, JJ, Tyndall, GS (1999) Atmospheric chemistry and global change (Oxford University Press, New York).

[14] Solomon, S (1990) Progress towards a quantitative understanding of antarctic ozone depletion. Nature 347:347.

[15] Lu, QB, Sanche, L (2001) Effects of cosmic rays on atmospheric chlorofluorocarbon dissociation and ozone depletion. Phys. Rev. Lett. 87:078501.

[16] Wang, CR, Drew, K, Luo, T, Lu, MJ, Lu, QB (2008) Resonant dissociative electron transfer of the presolvated electron to $\mathrm{CCl}_{4}$ in liquid: Direct observation and lifetime of the $\mathrm{CCl}_{4}^{*-}$ transition state. J. Chem. Phys. 128:041102.

[17] Lu, QB (2010) Cosmic-ray-driven electron-induced reactions of halogenated molecules adsorbed on ice surfaces: Implications for atmospheric ozone depletion and global climate change. Phys. Rep. 487:141.

[18] Bertin, M et al. (2009) Reactivity of water-electron complexes on crystalline ice surfaces. Faraday Discuss. 141:293.

[19] Molina, MJ, Rowland, FS (1974) Stratospheric sink for chlorofluoromethanes: chlorine atom catalysed destruction of ozone. Nature 249:810.

[20] Lin, L, Balabin, I, Beratan, D (2005) The nature of aqueous tunneling pathways between electron-transfer proteins. Science 310:1311.

[21] Link, O (2007) Ph.D. thesis (Universität Göttingen).

[22] Link, O et al. (2009) Ultrafast electronic spectroscopy for chemical analysis near liquid water interfaces: concepts and applications. Appl. Phys. A 96:117.

[23] Link, O et al. (2009) Ultrafast phase transition in metastable water near liquid interfaces. Faraday Discuss. 141:67.

[24] Coe, JV (2001) Fundamental properties of bulk water from cluster ion data. Int. Rev. Phys. Chem. 20:33.

[25] Bragg, AE, Verlet, JRR, Kammrath, A, Cheshnovsky, O, Neumark, DM (2004) Hydrated electron dynamics: from clusters to bulk. Science 306:669.

[26] Verlet, JRR, Bragg, AE, Kammrath, A, Cheshnovsky, O, Neumark, DM (2005) Observation of large water-cluster anions with surface-bound excess electrons. Science 307:93. 
[27] Coe, JV, Arnold, ST, Eaton, JG, Lee, GH, Bowen, KH (2006) Photoelectron spectra of hydrated electron clusters: Fitting line shapes and grouping isomers. J. Chem. Phys. 125:014315.

[28] Coe, JV, Williams, SM, Bowen, KH (2008) Photoelectron spectra of hydrated electron clusters vs. cluster size: connecting to bulk. Int. Rev. Phys. Chem. $27: 27$.

[29] Neumark, D (2008) Spectroscopy and dynamics of excess electrons in clusters. Mol. Phys. 106:2183.

[30] Ma, L, Majer, K, Chirot, F, von Issendorff, B (2009) Low temperature photoelectron spectra of water cluster anions. J. Chem. Phys. 131:144303.

[31] Barnett, RN, Landman, U, Cleveland, CL, Jortner, J (1988) Electron localization in water clusters. II. surface and internal states. J. Chem. Phys. 88:4429.

[32] Turi, L, Sheu, WS, Rossky, PJ (2005) Characterization of excess electrons in water-cluster anions by quantum simulations. Science 309:914.

[33] Frigato, T, VandeVondele, J, Schmidt, B, Schütte, C, Jungwirth, P (2008) $\mathrm{Ab}$ initio molecular dynamics simulation of a medium-sized water cluster anion: From an interior to a surface-located excess electron via a delocalized state. J. Phys. Chem. A 112:6125.

[34] Madarasz, A, Rossky, PJ, Turi, L (2009) Interior- and surface-bound excess electron states in large water cluster anions. J. Chem. Phys. 130:124319.

[35] Madarasz, A, Rossky, PJ, Turi, L (2010) Response of observables for cold anionic water clusters to cluster thermal history. J. Phys. Chem. A 114:2331.

[36] Marsalek, O, Uhlig, F, Frigato, T, Schmidt, B, Jungwirth, P (2010) Dynamics of electron localization in warm vs. cold water clusters. submitted.

[37] Weyl, W (1864) Ueber Metallammonium-Verbindungen. Ann. Phys. Leipzig 197:601.

[38] Kraus, CA (1908) Solutions of metals in non-metallic solvents, VI material effects accompanying the passage of an electrical current through solutions of metals in liquid ammonia, migration experiments. J. Am. Chem. Soc. 30:1323.

[39] Kevan, L (1978) Localization and solvation of electrons in condensed media, the simplest radical anion? J. Phys. Chem. 82:1144. 
[40] Rossky, PJ, Schnitker, J (1988) The hydrated electron: quantum simulation of structure, spectroscopy, and dynamics. J. Phys. Chem. 92:4277.

[41] Migus, A, Gauduel, Y, Martin, JL, Antonetti, A (1987) Excess electrons in liquid water: First evidence of a prehydrated state with femtosecond lifetime. Phys. Rev. Lett. 58:1559.

[42] Kimura, Y, Alfano, JC, Walhout, PK, Barbara, PF (1994) Ultrafast transient absorption spectroscopy of the solvated electron in water. J. Phys. Chem. 98:3450.

[43] Yokoyama, K, Silva, C, Son, DH, Walhout, PK, Barbara, PF (1998) Detailed investigation of the femtosecond pump-probe spectroscopy of the hydrated electron. J. Phys. Chem. A 102:6957.

[44] Assel, M, Laenen, R, Laubereau, A (1998) Dynamics of excited solvated electrons in aqueous solution monitored with femtosecond-time and polarization resolution. J. Phys Chem. A 102:2256.

[45] Shi, X, Long, FH, Lu, H, Eisenthal, KB (1996) Femtosecond electron solvation kinetics in water. J. Phys. Chem. 100:11903.

[46] Pshenichnikov, MS, Baltuska, A, Wiersma, DA (2004) Hydrated-electron population dynamics. Chem. Phys. Lett. 389:171.

[47] Elles, CG, Jailaubekov, AE, Crowell, RA, Bradforth, SE (2006) Excitationenergy dependence of the mechanism for two-photon ionization of liquid $\mathrm{H}_{2} \mathrm{O}$ and $\mathrm{D}_{2} \mathrm{O}$ from 8.3 to $12.4 \mathrm{eV}$. J. Chem. Phys. 125:044515.

[48] Laenen, R, Roth, T, Laubereau, A (2000) Novel precursors of solvated electrons in water: Evidence for a charge transfer process. Phys. Rev. Lett. $85: 50$.

[49] Lian, R, Oulianov, DA, Shkrob, IA, Crowell, RA (2004) Geminate recombination of electrons generated by above-the-gap $(12.4 \mathrm{eV})$ photoionization of liquid water. Chem. Phys. Lett. 398:102.

[50] Sander, MU, Gudiksen, MS, Luther, K, Troe, J (2000) Liquid water ionization: mechanistic implications of the $\mathrm{H} / \mathrm{D}$ isotope effect in the geminate recombination of hydrated electrons. Chem. Phys. 258:257.

[51] Bartels, DM, Crowell, RA (2000) Photoionization yield vs energy in $\mathrm{H}_{2} \mathrm{O}$ and $\mathrm{D}_{2} \mathrm{O}$. J. Phys. Chem. A 104:3349.

[52] Crowell, RA, Bartels, DM (1996) Multiphoton ionization of liquid water with 3.0-5.0 eV photons. J. Phys. Chem. 100:17940. 
[53] Sander, MU, Luther, K, Troe, J (1993) On the photoionization mechanism of liquid water. Ber. Bunsen Ges. Phys. Chem. 97:953.

[54] Sander, MU, Luther, K, Troe, J (1993) Excitation-energy dependence of the photoionization of liquid water. J. Phys. Chem. 97:11489.

[55] Nikogosyan, N, Oraevsky, AA, Rupasov, VI (1983) two-photon ionization and dissoziation of liquid water by powerful laser UV radiation. Chem. Phys. $77: 131$.

[56] Elles, CG, Shkrob, IA, Crowell, RA, Bradforth, SE (2007) Excited state dynamics of liquid water: Insight from the dissociation reaction following two-photon excitation. J. Chem. Phys. 126:164503.

[57] Shirom, M, Stein, G (1969) The absorption spectrum of the ferrocyanide ion in aqueous solution. Isr. J. Chem. 7:405.

[58] Shirom, M, Stein, G (1971) Excited state chemistry of the ferrocyanide ion in aqueous solution. I. formation of the hydrated electron. J. Chem. Phys. $55: 3372$.

[59] Lenchenkov, V, Kloepfer, J, Vilchiz, V, Bradforth, SE (2001) Electron photodetachment from $\left[\mathrm{Fe}(\mathrm{CN})_{6}\right]^{4-}$ : photoelectron relaxation and geminate recombination. Chem. Phys. Lett. 342:277.

[60] Chen, X, Bradforth, SE (2008) The ultrafast dynamics of photodetachment. Annu. Rev. Phys. Chem. 59:203.

[61] Armbruster, M, Haberland, H, Schindler, HG (1981) Negatively charged water clusters, or the first observation of free hydrated electrons. Phys. Rev. Lett. $47: 323$.

[62] Ayotte, P, Johnson, MA (1977) Electronic absorption spectra of size-selected hydrated electron clusters: $\left(\mathrm{H}_{2} \mathrm{O}\right)_{\mathrm{n}}^{-}, \mathrm{n}=6-50$. J. Chem. Phys. 106:811.

[63] Hammer, NI et al. (2004) How do small water clusters bind an excess electron? Science 306:675.

[64] Hammer, NI, Roscioli, JR, Bopp, JC, Headrick, JM, Johnson, MA (2005) Vibrational predissociation spectroscopy of the $\left(\mathrm{H}_{2} \mathrm{O}\right)_{6-21}^{-}$clusters in the oh stretching region: evolution of the excess electron binding signature into the intermediate cluster size regime. J. Chem. Phys. 123:244311.

[65] Hammer, NI, Roscioli, JR, Johnson, MA (2005) Identification of two distinct electron binding motifs in the anionic water clusters: A vibrational spectroscopic study of the $\left(\mathrm{H}_{2} \mathrm{O}\right)_{6}^{-}$isomers. J. Phys Chem. A 109:7896. 
[66] Roscioli, JR, Hammer, NI, Johnson, MA (2006) Infrared spectroscopy of water cluster anions, $\left(\mathrm{H}_{2} \mathrm{O}\right)_{\mathrm{n}=3-24}^{-}$in the $\mathrm{HOH}$ bending region: Persistence of the double $\mathrm{H}$-bond acceptor (AA) water molecule in the excess electron binding site of the class I isomers. J. Phys Chem. A 110:7517.

[67] Roscioli, JR, Johnson, MA (2007) Isomer-specific spectroscopy of the $\left(\mathrm{H}_{2} \mathrm{O}\right)_{8}^{-}$ cluster anion in the intramolecular bending region by selective photodepletion of the more weakly electron binding species (isomer II). J. Chem. Phys. 126:024307.

[68] Roscioli, JR, Hammer, NI, Johnson, MA, Diri, K, Jordan, KD (2008) Exploring the correlation between network structure and electron binding energy in the $\left(\mathrm{H}_{2} \mathrm{O}\right)_{7}^{-}$cluster through isomer-photoselected vibrational predissociation spectroscopy and ab initio calculations: addressing complexity beyond types I-III. J. Chem. Phys. 128:104314.

[69] Asmis, KR et al. (2007) Infrared multiple photon dissociation of the hydrated electron clusters $\left(\mathrm{H}_{2} \mathrm{O}\right)_{15-50}^{-}$. J. Chem. Phys. 126:191105.

[70] Coe, JV et al. (1990) Photoelectron spectroscopy of hydrated electron cluster anions, $\left(\mathrm{H}_{2} \mathrm{O}\right)_{\mathrm{n}=2-69}^{-}$. J. Chem. Phys. 92:3980.

[71] Kim, J, Becker, L, Cheshnovsky, O, Johnson, MA (1998) Photoelectron spectroscopy of the missing hydrated electron clusters $\left(\mathrm{H}_{2} \mathrm{O}\right)_{\mathrm{n}}^{-}, \mathrm{n}=3,5,8$ and 9: isomers and continuity with the dominant clusters $\mathrm{n}=6,7$ and $\geq 11$. Chem. Phys. Lett. 297:90.

[72] Kammrath, A, Verlet, JRR, Griffin, GB, Neumark, DM (2006) Photoelectron spectroscopy of large $\left(\mathrm{H}_{2} \mathrm{O}\right)_{\mathrm{n}}^{-}(\mathrm{n}=50-200)$ clusters at $4.7 \mathrm{eV}$. J. Chem. Phys. 125:076101.

[73] Paik, DH, Lee, IR, Yang, DS, Baskin, JS, Zewail, AH (2004) Electrons in finite-sized water cavities: hydration dynamics observed in real time. Science 306:672.

[74] Sommerfeld, T, Jordan, KD (2005) Quantum drude oscillator model for describing the interaction of excess electrons with water clusters: An application to $\left(\mathrm{H}_{2} \mathrm{O}\right)_{1} 3^{-}$. J. Phys Chem. A 109:11531.

[75] Sommerfeld, T, Gardner, S, DeFusco, A, Jordan, KD (2006) Low-lying isomers and finite temperature behavior of $\left(\mathrm{H}_{2} \mathrm{O}\right)_{6}^{-}$. J. Chem. Phys. 125:174301.

[76] Sommerfeld, T, Jordan, KD (2006) Electron binding motifs of $\left(\mathrm{H}_{2} \mathrm{O}\right)_{\mathrm{n}}^{-}$ clusters. J. Am. Chem. Soc. 128:5828. 
[77] Herbert, JM, Head-Gordon, M (2005) Calculation of electron detachment energies for water cluster anions: An appraisal of electronic structure methods, with application to $\left(\mathrm{H}_{2} \mathrm{O}\right)_{20}^{-}$and $\left(\mathrm{H}_{2} \mathrm{O}\right)_{24}^{-}$. J. Phys Chem. A 109:5217.

[78] Herbert, JM, Head-Gordon, M (2006) Charge penetration and the origin of large O-H vibrational red-shifts in hydrated-electron clusters, $\left(\mathrm{H}_{2} \mathrm{O}\right)_{\mathrm{n}}^{-}$. J. Am. Chem. Soc. 128:13932.

[79] Herbert, JM, Head-Gordon, M (2006) First-principles, quantum-mechanical simulations of electron solvation by a water cluster. Proc. Natl. Acad. Sci. 103:14282.

[80] Khan, A (2006) Ab initio studies on $\left(\mathrm{H}_{2} \mathrm{O}\right)_{14}^{-}$clusters: existence of surface and interior-bound extra electrons. J. Chem. Phys. 125:024307.

[81] Khan, A (2004) Extra electron in $\left(\mathrm{H}_{2} \mathrm{O}\right)_{24}^{-}$cluster isomers: a theoretical study. J. Chem. Phys. 121:280.

[82] Khan, A (2008) Selecting high stability water clusters by examining locations of free $\mathrm{OH}$ bonds: application in the study of $\left(\mathrm{H}_{2} \mathrm{O}\right)_{18}^{-}$clusters. J. Mol. Struct. THEOCHEM 850:144.

[83] Verlet, JRR, Bragg, AE, Kammrath, A, Cheshnovsky, O, Neumark, DM (2005) Comment on "characterization of excess electrons in water-cluster anions by quantum simulations". Science 310:1769b.

[84] Turi, L, Sheu, WS, Rossky, PJ (2005) Response to comment on "characterization of excess electrons in water-cluster anions by quantum simulations". Science 310:1769.

[85] Madarasz, A, Rossky, PJ, Turi, L (2007) Exess electron relaxation dynamics at water/air interfaces. J. Chem. Phys. 126:234707.

[86] Baletto, F, Cavazzoni, C, Scandolo, S (2005) Surface trapped excess electrons on ice. Phys. Rev. Lett. 95:176801.

[87] Hermann, A, Schwerdtfeger, P, Schmidt, WG (2008) Theoretical study of the localization of excess electrons at the surface of ice. J. Phys. Condens. Matter 20:225003.

[88] Meyer, M, Stähler, J, Kusmierek, D, Wolf, M, Bovensiepen, U (2008) Determination of the electrons solvation site on $\mathrm{D}_{2} \mathrm{O} / \mathrm{Cu}(111)$ using $\mathrm{Xe}$ overlayers and femtosecond photoelectron spectroscopy. Phys. Chem. Chem. Phys. 10:4932. 
[89] Bovensiepen, U et al. (2009) A dynamic landscape from femtoseconds to minutes for excess electrons at ice-metal interfaces. J. Phys. Chem. C 113:979.

[90] Schuster, GB (2000) Long-range charge transfer in DNA: Transient structural distortions control the distance dependence. Acc. Chem. Res. 33:253.

[91] Aflatooni, K, Gallup, GA, Burrow, PD (1998) Electron attachment energies of the DNA bases. J. Phys Chem. A 102:6205.

[92] Martin, F et al. (2004) DNA strand breaks induced by 0-4 eV electrons: the role of shape resonances. Phys. Rev. Lett. 93:068101.

[93] Gu, J, Xie, Y, Schaefer, III, HF (2006) Near 0 eV electrons attach to nuleiotides. J. Am. Chem. Soc. 128:1250.

[94] Gu, J, Xie, Y, Schaefer, III, HF (2006) Electron attachment to nuleotides in aqueous solution. Chem. Phys. Chem. 7:1885.

[95] Gu, J, Xie, Y, Schaefer, III, HF (2007) Electron attachment to DNA single strands: gas phase and aqueous solution. Nuclei Acids Res. 35:5165.

[96] Gu, J, Xie, Y, Schaefer, III, HF (2010) Electron attachment to oligonucleotide dimers in water: microsolvation-assisted base-stacking forms. Chem. Phys. Lett. 473:213.

[97] Lyngdoh, RHD, Schaefer, III, HF (2009) Elementary lesions in DNA subunits: Electron, hydrogen atom, proton, and hydride transfers. Acc. Chem. Res. $42: 563$.

[98] Gu, J, Xie, Y, Schaefer, III, HF (2010) Guanine nucleotides: base-centered and phosphate-centered valence-bound radical anions in aqueous solution. $J$. Phys. Chem. B 114:1221.

[99] Hendricks, JH, Lyapustina, SA, deClercq, HL, Bowen, KH (1998) The dipole bound-to-covalent anion transformation in uracil. J. Chem. Phys. 108:8.

[100] Schiedt, J, Weinkauf, P, Neumark, DM, Schlag, EW (1998) Anion spectroscopy of uracil, thymine and the amino-oxo and amino-hydroxy tautomers of cytosine and their water clusters. Chem. Phys. 239:511.

[101] Ito, T, Baker, SC, Stickley, CD, Peak, JG, Peak, MJ (1993) Dependence of the yield of strand breaks induced by $\gamma$-rays in DNA on the physical conditions of exposure: water content and temperature. J. Int. Radiat. Biol. 63:289. 
[102] Wang, CR, Luo, T, Lu, QB (2008) On the liftimes and phyiscal nature of incompletely relaxed electrons in liquid water. Phys. Chem. Chem. Phys. 10:4463.

[103] Lu, QB, Madey, TE (1999) Giant enhancement of electron-induced dissociation of chlorofluorocarbons coadsorbed with water or ammonia ices: implications for atmospheric chemistry. J. Chem. Phys. 111:2861.

[104] Ryu, S, Chang, J, Kwon, H, Kim, SK (2006) Dynamics of solvated electron transfer in thin ice film leading to a large enhancement in photodissociation of $\mathrm{CFCl}_{3}$. J. Am. Chem. Soc. 128:3500.

[105] Müller, R (2003) Impact of cosmic rays on stratospheric chlorine chemistry and ozone depletion. Phys. Rev. Lett. 91:058502.

[106] Lu, QB (2009) Correlation between cosmic rays and ozone depletion. Phys. Rev. Lett. 102:118501.

[107] von Benten, R (2006) Ph.D. thesis (Universität Göttingen).

[108] Krausz, F, Ivanov, M (2009) Attosecond physics. Rev. Mod. Phys. 81:163.

[109] Kling, MF et al. (2006) Control of electron localization in molecular dissociation. Science 312:246.

[110] Eden, JG (2004) High-order harmonic generation and other intense optical field-matter interactions: review of recent experimental and theoretical advances. Prog. Quantum Electron. 28:197.

[111] Hentschel, M et al. (2001) Attosecond metrology. Nature 414:509.

[112] Corkum, PB (1993) Plasma perspective on strong-field multiphoton ionization. Phys. Rev. Lett. 71:1994.

[113] Agostini, P, Fabre, F, Mainfray, G, Petite, G, Rahman, NK (1979) Free-free transitions following 6-photon ionization of xenon atoms. Phys. Rev. Lett. 42:1127.

[114] Fittinghoff, DN, Bolton, PR, Chang, B, Kulander, KC (1992) Observation of nonsequential double ionization of helium with optical tunneling. Phys. Rev. Lett. 69:2642.

[115] Lewenstein, M, Balcou, P, Ivanov, MY, LHuiller, A, Corkum, PB (1994) Theory of high-harmonic generation by low-frequency laser fields. Phys. Rev. A 49:2117. 
[116] Krause, JL, Schafer, KJ, Kulander, KC (1992) High-order harmonic generation from atoms and ions in the high intensity regime. Phys. Rev. Lett. 68:3535.

[117] Huefner, S (2003) Photoelectron spectroscopy (Springer-Verlag Berlin Heidelberg).

[118] Fellner-Feldegg, H, Siegbahn, H, Asplund, L, Kelfve, P, Siegbahn, K (1975) ESCA applied to liquids IV. a wire system for ESCA measurements on liquids. J. Electron Spectrosc. Relat. Phenom. 7:421.

[119] Siegbahn, H (1985) Electron spectroscopy for chemical analysis of liquids and solutions. J. Phys. Chem. 89:897.

[120] Lundholm, M, Siegbahn, H, Holmberg, S, Arbman, M (1986) Core electron spectroscopy of water solutions. J. Electron. Spectrosc. Relat. Phenom. 40:163.

[121] Faubel, M, Kisters, T (1989) Non-equilibrium molecular evaporation of carboxylic acid dimers. Nature 339:527.

[122] Faubel, M, Steiner, B, Toennies, JP (1997) Photoelectron spectroscopy of liquid water, some alcohols, and pure nonane in free micro jets. J. Chem. Phys. 106:9013.

[123] Winter, B, Faubel, M (2006) Photoemission from liquid aqueous solutions. Chem. Rev. 106:1176.

[124] Winter, B et al. (2006) Electron binding energies of hydrated $\mathrm{H}_{3} \mathrm{O}^{+}$and $\mathrm{OH}^{-}$: Photoelectron spectroscopy of aqueous acid and base solutions combined with electronic structure calculations. J. Am. Chem. Soc. 128:3864.

[125] Winter, B, Aziz, EF, Hergenhahn, U, Faubel, M, Hertel, I (2007) Hydrogen bonds in liquid water studied by photoelectron spectroscopy. J. Chem. Phys. 126:124504.

[126] Slavicek, P, Winter, B, Faubel, M, Bradforth, SE, Jungwirth, P (2009) Ionization energies of aqueous nucleic acids: Photokelectron spectroscopy of pyrimidine nucleosides and ab initio calculations. J. Am. Chem. Soc. 131:6460.

[127] Faubel, M (2000) Photoionization and Photodetachment (C. Y. Ng, World Scientific).

[128] Faubel, M (2009) private communication. 
[129] Braslau, A, Pershan, PS, Swislow, G, Ocko, BM, Als-Nielsen, J (1988) Capillary waves on the surface of simple liquids measured by X-ray reflectivity. Phys. Rev. A 38:2457.

[130] Schwartz, DK et al. (1990) Thermal diffuse X-ray-scattering studies of the water-vapor interface. Phys. Rev. A 41:5687.

[131] Kuo, IFW, Mundy, CJ (2004) An ab initio molecular dynamics study of the aqueous liquid-vapor interface. Science 303:658.

[132] Siefermann, K (2007) Master's thesis (Universität Karlsruhe (TH)).

[133] Nordlund, D et al. (2008) Electronic structure effects in liquid water studied by photoelectron spectroscopy and density functional theory. Chem. Phys. Lett. 460:86.

[134] Brundle, CR, Turner, DW (1968) High resolution molecular photoelectron spectroscopy, II. Water and deuterium oxide. Proc. Roy. Soc. A. 307:27.

[135] Truong, SY et al. (2009) Threshold photoelectron spectroscopy of $\mathrm{H}_{2} \mathrm{O}$ and $\mathrm{D}_{2} \mathrm{O}$ over the photon energy range 12-40 eV. Chem. Phys. 355:183.

[136] Weber, R (2003) Ph.D. thesis (Freie Universität Berlin).

[137] Winter, B et al. (2004) Full valence band photoemission from liquid water using EUV synchrotron radiation. J. Phys. Chem. A 108:2625.

[138] do Couto, PC, Estacio, SG, Cabral, BJC (2005) The Kohn-Sham density of states and band gap of water: From small clusters to liquid water. J. Chem. Phys. 123:054510.

[139] Pianetta, P (2009) X-ray data booklet, http://cxro.lbl.gov/xdb.

[140] Ottosson, N, Faubel, M, Bradforth, SE, Jungwirth, P, Winter, B (2010) Photoelectron spectroscopy of liquid water and aqueous solution: Electron effective attenuation lengths and emission-angle anisotropy. J. Electron. Spectrosc. Relat. Phenom. 177:60.

[141] Emfietzoglou, D, Nikjoo, H (2005) The effect of model approximations on single-collision distributions of low-energy electrons in liquid water. Radiat. Res. 163:98.

[142] Michaud, M, Wen, A, Sanche, L (2003) Cross sections for low-energy (1-100 $\mathrm{eV}$ ) electron elastic and inelastic scattering in amorphous ice. Radiat. Res. 159:3. 
[143] Mata, RA, Stoll, H, Costa-Cabral, BJ (2009) A simple one-body approach to the calculation of the first electronic absorption band of water. J. Chem. Theory Comput. 5:1829.

[144] Nordlund, D et al. (2007) Probing the electron delocalization in liquid water and ice at attosecond time scales. Phys. Rev. Lett. 99:217406.

[145] Peterson, P, Saykally, R (2005) Adsorption of ions to the surface of dilute electrolyte solutions: The jones-ray effect revisited. J. Am. Chem. Soc. 44:15446.

[146] Lian, R et al. (2006) Electron photodetachment from aqueous anions. 3. Dynamics of geminate pairs derived from photoexcitation of mono- vs polyatomic anions. J. Phys. Chem. A 110:9071.

[147] Bonin, J, Lampre, I, Mostafavi, M (2005) Absorption spectrum of the hydrated electron paired with nonreactive metal cations. Rad. Phys. Chem. 74:288-296.

[148] Spezia, R, Nicolas, C, Archirel, P, Boutin, A (2004) Molecular dynamics simulations of the $\mathrm{Ag}^{+}$or $\mathrm{Na}^{+}$cation with an excess electron in bulk water. J. Chem. Phys. 120:5261.

[149] Tang, Y et al. (2010) Direct measurement of vertical binding energy of a hydrated electron. Phys. Chem. Chem. Phys. 12:3653.

[150] Lin, M et al. (2007) Temperature effect on the absorption spectrum of the hydrated electron paired with a lithium cation in deuterated water. J. Phys. Chem. A 111:3548.

[151] Schmidt, KH, Han, P, Bartels, DM (1992) Temperature dependence of solvated electron diffusion in $\mathrm{H}_{2} \mathrm{O}$ and $\mathrm{D}_{2} \mathrm{O}$. J. Phys. Chem. 96:199.

[152] Schmidt, KH, Han, P, Bartels, DM (1995) Radiolytic yields of the hydrated electron from transient conductivity: improved calculation of the hydrated electron diffusion coefficient and analysis of some diffusion-limited $\left(\mathrm{e}_{\mathrm{aq}}^{-}\right)$reaction rates. J. Phys. Chem. 99:10530.

[153] Tay, KA, Boutin, A (2009) Hydrated electron diffusion: The importance of hydrogen-bond dynamics. J. Phys. Chem. B 113:11943.

[154] Smith, JD et al. (2004) Energetics of hydrogen bond network rearrangements in liquid water. Science 306:851.

[155] Nilsson, A et al. (2005) Comment on "energetics of hydrogen bond network rearrangements in liquid water". Science 308:793a. 
[156] Smith, JD, Cappa, CD, Messer, BM, Cohen, RC, Saykally, RJ (2005) Response to comment on "energetics of hydrogen bond network rearrangements in liquid water". Science 308:793b.

[157] Knipping, EM et al. (2000) Experiments and simulations of ion-enhanced interfacial chemistry on aqueous $\mathrm{NaCl}$ aerosols. Science 288:301.

[158] Reeser, DI et al. (2009) Photoenhanced reaction of ozone with chlorophyll at the seawater surface. J. Phys. Chem. C 113:2071.

[159] Wei, X, Miranda, PB, Shen, YR (2001) Surface vibrational spectroscopic study of surface melting of ice. Phys. Rev. Lett. 86:1554.

[160] Poterya, V, Farnik, M, Slavicek, P, Buck, U, Kresin, VV (2007) Photodissociation of hydrogen halide molecules on free ice nanoparticles. J. Chem. Phys. 126:071101.

[161] Sevilla, MD, Summerfield, S, Eliezer, I, Rak, J, Symons, MCR (1997) Interaction of the chlorine atom with water: ESR and ab initio MO evidence for three-electron $\left(\sigma 2 \sigma^{*} 1\right)$ bonding. J. Phys. Chem. A 101:2910.

[162] Sagar, DM, Bain, CD, Verlet, JRR (2010) Hydrated electrons at the water/air interface. J. Am. Chem. Soc. 132:6917.

[163] Lee, YJ, Kee, TW, Zhang, T, Barbara, PF (2004) Pump-probe spectroscopy of the hydrated electron in reverse micelles. J. Phys. Chem. B 108:3474.

[164] Bragg, AE, Verlet, JRR, Kammrath, A, Cheshnovsky, O, Neumark, DM (2005) Electronic relaxation dynamics of water cluster anions. J. Am. Chem. Soc. 127:15283.

[165] Baum, G, Huber, JR (1993) Photodissociation of $\mathrm{CF}_{2} \mathrm{Cl}_{2}$ at $193 \mathrm{~nm}$ investigated by photofragment translational spectroscopy. Chem. Phys. Lett. 203:261. 
In order to be irreplaceable one must always be different!

Coco Chanel 




\section{KATRIN R. SIEFERMANN}

PERSONAL

Name:

Date of Birth:

Place of Birth:

Nationality:
Katrin Rita Siefermann

September 10, 1982

Oberkirch, Germany

German

ACADEMIC TRACK RECORD Hans-Furler Gymnasium

1993-2002

Oberkirch, Germany

Student at the Universität Karlsruhe (TH)

Oct 2002 - Oct 2006

Faculty of Chemistry

Major: Physical Chemistry

Diploma thesis at the Georg-August-Universität Göttingen

Nov 2006 - Mav 2007 Faculty of Chemistry, Department of Physical Chemistry

Title: "Zeitaufgelöste Photoelektronenspektroskopie am Microwasserstrahl"

("Time-resolved photoelectron spectroscopy on a liquid water micro-jet")

Research stay at the University of Wisconsin-Madison

fune 2007 - Oct 2007

in the group of Prof. F. Fleming Crim.

Topic: Photoisomerization dynamics of cis-stilbene in solution

Ph.D. thesis at the Georg-August-Universität Göttingen

Nov 2007 - July 2010

in the group of Prof. Bernd Abel.

Title: "Ultrafast photoelectron spectroscopy near liquid water interfaces:

The solvated electron"

Research stay with Prof. Daniel Neumark

Nov 2009

at the University of California Berkeley.

Research stay with Dr. Michal Farnik

Dec 2009

at the J. Heyrovsky Institute of Physical Chemistry, Prague.

AWARDS \& FELLOWSHIPS * Procter \& Gamble award 2008.

* Ph.D. scholarship by the Physical \& Chemical Graduate School Göttingen (GRK 782).

* Travel scholarship by the „Studienstiftung des deutschen Volkes“ (German National

Academic Foundation) for my research stay at the University of Wisconsin-Madison.

* Scholarship by the „Studienstiftung des deutschen Volkes“.

* Scholarship by the „Fonds des Verbandes der Chemischen Industrie“.

* Participation in the 59th Meeting of Nobel Laureates at Lindau (2009). 